\title{
Optimal transportation networks as flat chains
}

\author{
EMANUELE PAOLINI ${ }^{\dagger}$ \\ Dipartimento di Matematica "U. Dini”, Università di Firenze, \\ viale Morgagni 67/A, 50134 Firenze, Italy \\ AND \\ EUGENE STEPANOV \\ Dipartimento di Matematica “L. Tonelli”, Università di Pisa, \\ via Buonarroti 2, 56127 Pisa, Italy
}

[Received 30 May 2005 and in revised form 24 June 2006]

\begin{abstract}
We provide a model of optimization of transportation networks (e.g. urban traffic lines, subway or railway networks) in a geographical area (e.g. a city) with given density of population and that of services and/or workplaces, the latter being the destinations of everyday movements of the former. The model is formulated in terms of the Federer-Fleming theory of currents, and allows us to get both the position and the necessary capacity of the optimal network. Existence and some qualitative properties of solutions to the relevant optimization problem are studied. Also, in an important particular case it is shown that the model proposed is equivalent to another known model of optimization of a transportation network, the latter not using the language of currents.
\end{abstract}

\section{Introduction}

Let $\varphi^{+}, \varphi^{-}$stand for finite Borel measures with compact support in $\mathbb{R}^{n}$ and of equal total mass $\varphi^{+}\left(\mathbb{R}^{n}\right)=\varphi^{-}\left(\mathbb{R}^{n}\right)$, the former representing the density of population, the latter the density of workplaces or services in some geographical area (e.g. a city). The aim of this paper is to provide a reasonable model of choosing a "transportation network" (e.g. the set of subway, or, generally speaking, urban traffic lines) in a city characterized by the distributions $\varphi^{ \pm}$. The network to be chosen has to facilitate the transportation of the population to the services. The model we consider is based primarily on the Monge-Kantorovich theory of optimal mass transport, but is expressed in terms of the Federer-Fleming theory of currents. Apart from the fact that the language of currents, as we will show later, is extremely natural for such urban planning problems, it also allows one to formulate the models which take into consideration the degree to which the pattern of behaviour of the population is "individualistic". Such models allow one as well to find naturally not only the position of the network to be constructed, but also the network capacity which is intrinsic in the model. Below we discuss in more detail the formulations of the models studied in this paper.

\footnotetext{
${ }^{\dagger}$ E-mail: paolini@math.unifi.it

" Partially supported by the Italian government program "Incentivazione alla mobilità di studiosi stranieri e italiani residenti all'estero". E-mail: stepanov@spb.runnet.ru, e.stepanov@sns.it
} 


\subsection{Transport problems}

The classical Monge-Kantorovich optimal transportation problem consists in finding the "optimal" way of transporting $\varphi^{+}$to $\varphi^{-}$. One of the many equivalent formulations of the problem reads as follows [8, 1]: find a finite Borel measure $\mu_{\mathrm{opt}}$ (called a transport density) and a Borel measurable unit vectorfield $v_{\mathrm{opt}}$ in $\mathbb{R}^{n}$ (called a field of transportation directions) which minimizes the total mass $\mu\left(\mathbb{R}^{n}\right)$ among all couples $(\mu, v)$ as above satisfying the Monge-Kantorovich transport equation

$$
\operatorname{div} \mu \nu=\varphi^{+}-\varphi^{-}
$$

in the sense of distributions.

One might easily reformulate this problem using the language of the Federer-Fleming theory of currents. In this case we identify $\varphi^{ \pm}$with zero-dimensional flat chains. The corresponding formulation would read: find a flat chain $T_{\mathrm{opt}}$ minimizing the total mass $\mathbb{M}(T)$ among all onedimensional real flat chains $T$ satisfying

$$
\partial T=\varphi^{+}-\varphi^{-}
$$

Clearly, once one finds $T_{\mathrm{opt}}=\tau_{T_{\mathrm{opt}}} \wedge \mu_{T_{\mathrm{opt}}}$, i.e. $\tau_{T_{\mathrm{opt}}}$ is the orientation of $T_{\mathrm{opt}}$ while $\mu_{T_{\mathrm{opt}}}$ is the underlying measure, one gets $\mu_{\mathrm{opt}}=\mu_{T_{\mathrm{opt}}}$ and $\nu_{\mathrm{opt}}=\tau_{T_{\mathrm{opt}}}$, and, vice versa, if one knows $\left(\mu_{\mathrm{opt}}, v_{\mathrm{opt}}\right)$, then one gets $T_{\mathrm{opt}}=v_{\mathrm{opt}} \wedge \mu_{\mathrm{opt}}$.

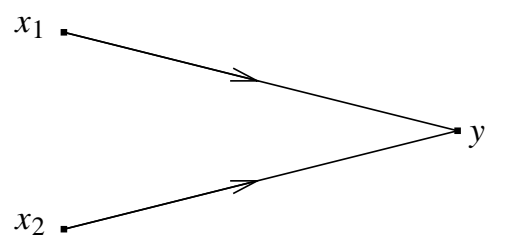

(a)

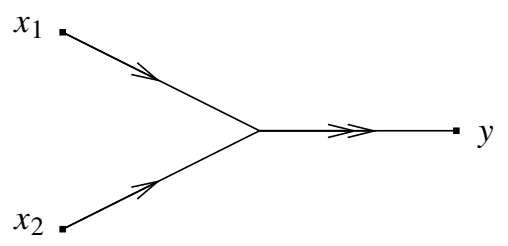

(b)

FIG. 1. Solutions to the Monge-Kantorovich transport problem with $\varphi^{+}:=\delta_{x_{1}}+\delta_{x_{2}}$ and $\varphi^{-}:=2 \delta_{y}$ in the case (a) $\alpha=1$ and (b) $\alpha<1$.

The above Monge-Kantorovich problem formulated in terms of currents admits a far reaching generalization in which one minimizes a generic $\alpha$-mass $\mathbb{M}^{\alpha}$ (for some given $\alpha \in[0,1]$ ) of the current $T$ instead of $\mathbb{M}$. The general formulation reads as follows: find a flat chain $T_{\mathrm{opt}}$ minimizing the $\alpha$-mass $\mathbb{M}^{\alpha}(T)$ among all one-dimensional real flat chains $T$ of finite mass satisfying (1). The background idea of this generalization is given by the following example. Let $\varphi^{+}:=\delta_{x_{1}}+\delta_{x_{2}}$ be the sum of two Dirac masses and $\varphi^{-}:=2 \delta_{y}$ be just one Dirac mass where the points $x_{1}, x_{2}$ and $y$ are positioned as in Figure 1. Then the solution of the classical problem (i.e. with $\alpha=1$ ) is given by the one-dimensional real polyhedral chain in Figure 1 1 a), i.e. the transportation occurs along the segments connecting the source points $x_{1}$ and $x_{2}$ with the destination $y$. However, the solution for $\alpha<1$ looks as in Figure 1, b). In other words, the role of the parameter $\alpha$ is to make it more convenient for people leaving the source points to make together part of their trip to the destination instead of moving "individually". If one interprets the solution $T_{\mathrm{opt}}$ as an optimal transportation network which provides the movement of $\varphi^{+}$to $\varphi^{-}$, then clearly it contains information on both the directions of the movement and on the capacity of the network at each point. 
In discrete settings such models were introduced and studied for communication networks in [14], for pipelines in [6] and for drainage networks in [15]. They are quite natural in fluid mechanics (and therefore also in the traffic flow models based on it) when modeling the flow of liquids in tubes subject to Poiseuille's law which implies the increase of resistance as the tube becomes thinner [11, 16, 5, 4]. In continuous settings such models were introduced in [23] and in a different though equivalent formulation in [16].

\subsection{Optimal transportation networks}

We now propose a more general model for choosing the optimal transportation network. In fact, suppose that one has to provide a set of fast transportation routes (i.e. a subway and/or a set of urban transportation lines) in a given city. We henceforth call such routes a transportation network. The flow of people moving along these routes will be modelled by a one-dimensional real flat chain $S$, while the flow of people moving without the use of those routes will be modelled by a onedimensional real flat chain $T$. It is reasonable to suppose that the cost of using the transportation network (for example the time spent for the travel) is proportional to $\mathbb{M}^{\beta}(S)$ with coefficient $B \geqslant 0$ and with some given $\beta \in[0,1]$, while the cost of movement of the population without using the network is proportional to $\mathbb{M}^{\alpha}(T)$ with coefficient $A \geqslant 0$ and with some given $\alpha \in[0,1]$. Then the number

$$
W(T, S):=A \mathbb{M}^{\alpha}(T)+B \mathbb{M}^{\beta}(S)
$$

represents the overall cost of everyday movement of the population. Clearly, the parameters $\alpha$ and $\beta$ model the degree to which the behaviour of the population is "individualistic" (i.e. when both are equal to one, one may assume that the behaviour is completely individualistic, while the smaller they are, the more convenient it is for people to make part of their itinerary together). It is further reasonable to assume that the cost of construction of the transportation network depends only on $\mathbb{M}^{\delta}(S)$ (in simplest applications one would even have $\delta=0$, i.e. the cost of construction depends only on the length of the network) according to some given nondecreasing function $H: \mathbb{R}^{+} \rightarrow \mathbb{R}^{+}$. Therefore, the number

$$
\mathfrak{F}(T, S):=W(T, S)+H\left(\mathbb{M}^{\delta}(S)\right)
$$

represents the total expenses of everyday movement of the population together with the cost of building the transportation network. One also assumes that the total flow of the population $T+S$ transports $\varphi^{+}$to $\varphi^{-}$, that is,

$$
\partial(T+S)=\varphi^{+}-\varphi^{-} .
$$

The following quite natural minimization problem describes the optimal choice of the transportation network.

PRoblem 1 Find a couple $\left(T_{\text {opt }}, S_{\text {opt }}\right)$ of one-dimensional real flat chains minimizing $\mathfrak{F}$ among all couples $(T, S)$ of real one-dimensional flat chains of finite mass, satisfying (3).

Note that in the particular case $B \geqslant A$ Problem 1 reduces to the version of the MongeKantorovich problem studied in [23] and mentioned in Subsection 1.1. Some qualitative properties of particular solutions to such a transportation problem (namely those which can be obtained as limits of solutions to appropriate approximating discrete problems) have been studied in [25, 24].

In this paper we study the existence (Theorem 8.1 ) as well as qualitative properties of solutions of Problem 1 like acyclicity (Theorem 10.1), rectifiability (Theorem 10.2) and properties of the 
support (Theorem 10.4). In particular, we provide conditions under which the relevant solutions are rectifiable and have some subtler regularity properties, namely, when the current $S_{\text {opt }}$ representing the transportation network to be constructed may be represented as a rectifiable current concentrated on a closed set (which gives the position of the optimal network) and with an u.s.c. density (representing network capacity) strictly greater than some nonnegative threshold (Theorem 10.7).

As an illustration of the results obtained, we summarize in the theorem below the assertions regarding Problem 1 under particular, though rather general conditions on problem data, which we consider to be most interesting for applications.

TheOREM 1.1 Let $A>0, \alpha \in[0,1), B \geqslant 0, H:[0,+\infty) \rightarrow[0,+\infty)$ strictly increasing, strictly concave and unbounded (i.e. $H(l) \rightarrow+\infty$ as $l \rightarrow+\infty), \delta \in[0, \alpha)$. Let also $\beta \in[0, \alpha]$ with $A>B$ if $\beta=\alpha$. Finally, suppose $\varphi^{ \pm}$are finite positive Borel measures with compact support in $\mathbb{R}^{n}$ and such that $\varphi^{+}\left(\mathbb{R}^{n}\right)=\varphi^{-}\left(\mathbb{R}^{n}\right)$. Then Problem 1 admits a solution, i.e. there exists a pair $\left(T_{\mathrm{opt}}, S_{\mathrm{opt}}\right)$ of real one-dimensional flat chains which minimizes the functional

$$
\mathfrak{F}(T, S)=A \mathbb{M}^{\alpha}(T)+B \mathbb{M}^{\beta}(S)+H\left(\mathbb{M}^{\delta}(S)\right)
$$

among all pairs $(T, S)$ of flat chains with finite mass and such that $\partial(T+S)=\varphi^{+}-\varphi^{-}$. Moreover every such optimal pair $\left(T_{\mathrm{opt}}, S_{\mathrm{opt}}\right)$ enjoys the following properties:

(i) $S_{\text {opt }}$ is a rectifiable current representable as $S_{\text {opt }}=\theta_{S_{\text {opt }}} \llbracket \Sigma_{\text {opt }} \rrbracket$, where $\Sigma_{\text {opt }}$ is a compact and countably $\left(\mathcal{H}^{1}, 1\right)$-rectifiable set, and the density $\theta_{S_{\mathrm{opt}}}(x)$ is u.s.c. and satisfies $\inf _{x \in \Sigma_{\text {opt }}} \theta_{S_{\text {opt }}}(x)=\theta_{0}>0$.

(ii) $T_{\mathrm{opt}}$ is a rectifiable current disjoint from $S_{\mathrm{opt}}$ in the sense that the measures $\mu_{T_{\mathrm{opt}}}$ and $\mu_{S_{\mathrm{opt}}}$ are mutually singular. Moreover $\sup _{x \in \mathbb{R}^{n}} \theta_{T_{\mathrm{opt}}}(x) \leqslant \theta_{0}$, where $\theta_{T_{\mathrm{opt}}}$ is the density of $T_{\mathrm{opt}}$.

(iii) $T_{\mathrm{opt}}+S_{\mathrm{opt}}$ is acyclic.

Note that some of the above results are quite natural. In fact, consider for simplicity the case when $H(l)=C l$ for some constant $C>0$. Then it is easy to observe that Problem 1 reduces to the version of the Monge-Kantorovich problem from Subsection 1.1, but with the mass $\mathbb{M}^{g}$ instead of $\mathbb{M}^{\alpha}$, where

$$
g(t):=A t^{\alpha} \wedge\left(B t^{\beta}+C t^{\delta}\right) .
$$

In fact, if $R_{\text {opt }}$ is a flat chain solving this problem, then it is rectifiable due to the general rectifiability theorem from [22], hence $R_{\mathrm{opt}}=\theta \llbracket \Sigma \rrbracket$ for some countably $\left(\mathcal{H}^{1}, 1\right)$-rectifiable set $\Sigma$, and therefore

$$
\begin{aligned}
\mathbb{M}^{g}\left(R_{\mathrm{opt}}\right) & =A \int_{\{x \in \Sigma: \theta(x)<d\}} \theta^{\alpha} \mathrm{d} \mathcal{H}^{1}+B \int_{\{x \in \Sigma: \theta(x) \geqslant d\}} \theta^{\beta} \mathrm{d} \mathcal{H}^{1}+C \int_{\{x \in \Sigma: \theta(x) \geqslant d\}} \theta^{\delta} \mathrm{d} \mathcal{H}^{1} \\
& =A \mathbb{M}^{\alpha}(T)+B \mathbb{M}^{\beta}(S)+H\left(\mathbb{M}^{\delta}(S)\right)=\mathfrak{F}(T, S)
\end{aligned}
$$

where $d>0$ is the unique number such that $A d^{\alpha}=B d^{\beta}+C d^{\delta}$, and

$$
T:=R_{\mathrm{opt}\llcorner}\{x \in \Sigma: \theta(x)<d\}, \quad S:=R_{\mathrm{opt}\llcorner}\{x \in \Sigma: \theta(x) \geqslant d\} .
$$

Conversely, if a pair $(T, S)$ "almost" solves Problem 1 (in the sense that $\mathfrak{F}(T, S)$ is close to the infimum of $\mathfrak{F}$ on the class of admissible pairs of flat chains), then one can show that up to decreasing the functional $\mathfrak{F}$ even more, one may assume both $T$ and $S$ rectifiable, while denoting $R:=T+S$, one has $R=\theta \llbracket \Sigma \rrbracket$ for some countably $\left(\mathcal{H}^{1}, 1\right)$-rectifiable set $\Sigma$, and

$$
T=R\llcorner\{x \in \Sigma: \theta(x)<d\}, \quad S=R\llcorner\{x \in \Sigma: \theta(x) \geqslant d\}
$$


(of course, with technical details omitted for the moment), and hence

$$
\mathbb{M}^{g}(R)=\mathfrak{F}(T, S) .
$$

Thus once the existence of a minimizer $R_{\text {opt }}$ for $\mathbb{M}^{g}$ on the class of real one-dimensional flat chains $R$ of finite mass satisfying $\partial R=\varphi^{+}-\varphi^{-}$is established (which can be done, for instance, using the machinery developed in [23] for $\mathbb{M}^{\alpha}$ instead of $\mathbb{M}^{g}$ ), we get the existence of a solution $(T, S)$ to Problem 1 given by (4). The threshold $\theta_{0}$ from Theorem 1.1 is then given by $\theta_{0}:=d$. Of course, the qualitative properties of the solution (e.g. that $S$ may be assumed to be concentrated on a closed set and have an u.s.c. density) are slightly more delicate even in this simple case.

We also show that in the particular case when $\alpha=\beta=1$ and $\delta=0$ Problem 1 is naturally equivalent to a particular case of the problem studied in [9] of optimizing a transportation network under the condition that the prices per unit distance of travelling with and without the help of the transportation network are constant.

The background idea we use in most of the results is the representation of normal onedimensional currents through measures over the appropriately metrized set of Lipschitz-continuous paths in $\mathbb{R}^{n}$ (further called transports). The idea of using such a representation when dealing with one-dimensional currents goes back to [19], although in a different context such measures were already used in [7]. In the context of transportation and urban problems such measures were employed in [10, 9] and, implicitly, also in [16, 5]. In fact, the description of mass transportation through transports happens to be more precise than through currents.

\section{Notation and preliminaries}

\subsection{Measures}

Unless otherwise explicitly stated, all the measures we will be dealing with are nonnegative Borel measures on $\mathbb{R}^{n}$. We denote by $\phi \wedge \psi$ the maximum nonnegative Borel measure $\mu$ satisfying $\mu(e) \leqslant$ $\psi(e) \wedge \phi(e)$ for all Borel $e \subset \mathbb{R}^{n}$. If $\varphi$ is a signed measure, we denote by $\varphi^{ \pm}$its positive and negative parts respectively.

We will say that a sequence of signed Radon measures $\phi_{v}$ converges in the narrow sense to a signed Radon measure $\phi$ if $\int_{\mathbb{R}^{n}} f \mathrm{~d} \phi_{\nu} \rightarrow \int_{\mathbb{R}^{n}} f \mathrm{~d} \phi$ as $v \rightarrow \infty$ for every bounded continuous function $f: \mathbb{R}^{n} \rightarrow \mathbb{R}$.

Let $\Theta_{k}^{*}(\mu, x)$ and $\Theta_{k *}(\mu, x)$ stand for the upper and lower $k$-dimensional density of the measure $\mu$ at $x \in \mathbb{R}^{n}$, that is,

$$
\Theta_{k}^{*}(\mu, x):=\limsup _{\rho \rightarrow 0^{+}} \frac{\mu\left(B_{\rho}(x)\right)}{\omega_{k} \rho^{k}}, \quad \Theta_{k *}(\mu, x):=\liminf _{\rho \rightarrow 0^{+}} \frac{\mu\left(B_{\rho}(x)\right)}{\omega_{k} \rho^{k}} .
$$

\subsection{Currents}

For basic notation on currents we refer to [17, 18]. Here we recall rather briefly some principal facts we will use. We will always deal with real currents, i.e. currents with real coefficients. If $T$ is a current, for every open $U \subset \mathbb{R}^{n}$ we set

$$
\mu_{T}(U):=\sup \left\{T(\omega): \operatorname{supp} \omega \subset U,\|\omega\|_{L^{\infty}} \leqslant 1\right\} .
$$


We also set $\mathbb{M}(T):=\mu_{T}\left(\mathbb{R}^{n}\right)$ and call this quantity the mass of $T$. It is well known that if $\mathbb{M}(T)<$ $+\infty$, then $\mu_{T}$ defines a finite Radon measure and $T$ is representable as $T=\tau_{T} \wedge \mu_{T}$ for some unit simple $k$-vector field $\tau_{T}$, in the sense that

$$
T(\omega)=\int_{\mathbb{R}^{n}}\left\langle\tau_{T}(x), \omega(x)\right\rangle \mathrm{d} \mu_{T}(x)
$$

for every regular differential $k$-form $\omega$. In this case we set, for every $\theta \in L^{1}\left(\mathbb{R}^{n} ; \mu_{T}\right)$,

$$
T \wedge \theta(\omega):=\int_{\mathbb{R}^{n}} \theta(x)\left\langle\tau_{T}(x), \omega(x)\right\rangle \mathrm{d} \mu_{T}(x),
$$

and $T\left\llcorner A:=T \wedge 1_{A}\right.$, where $1_{A}$ stands for the characteristic function of a Borel set $A \subset \mathbb{R}^{n}$. We say that a sequence of currents $T_{v}$ converges to a current $T$ in the flat norm (written $T_{v} \stackrel{\mathcal{F}}{\rightarrow} T$ ) if $\mathbb{F}\left(T_{v}-T\right) \rightarrow 0$, where

$$
\mathbb{F}(T):=\inf \{\mathbb{M}(A)+\mathbb{M}(B): T=A+\partial B\} .
$$

Clearly, the topology induced by the flat norm is stronger than the weak topology of currents. We say that $T$ is a normal current if $\mathbb{M}(T)<+\infty$ and $\mathbb{M}(\partial T)<+\infty$, and $T$ is a flat chain if there is a sequence $\left\{T_{\nu}\right\}$ of normal currents such that $T_{v} \stackrel{\mathcal{F}}{\rightarrow} T$.

We call $T$ a rectifiable current if there exists a countably $\left(\mathcal{H}^{k}, k\right)$-rectifiable set $\Sigma \subset \mathbb{R}^{n}$ and a function $\theta \in L^{1}\left(\mathcal{H}^{k}\llcorner\Sigma)\right.$ (called the multiplicity or density of $T$ ) such that $T=\tau_{T} \wedge \theta \mathcal{H}^{k}\llcorner\Sigma$, while the unit simple $k$-vector $\tau_{T}: \Sigma \rightarrow \mathbb{R}^{n}$ is an orientation of $\Sigma$, in the sense that for $\mathcal{H}^{k}$-a.e. $x \in \Sigma$ the vector $\tau_{T}(x)$ defines the approximate tangent plane to $\Sigma$ at $x$. In this case we also write $T=\theta \llbracket \Sigma \rrbracket$ when an orientation on $\Sigma$ is prescribed. One can show that if $T$ is a flat chain with $\mathbb{M}(T)<+\infty$, then $T$ is a rectifiable current if and only if for some countably $\left(\mathcal{H}^{k}, k\right)$-rectifiable set $\Sigma \subset \mathbb{R}^{n}$ one has $T=T\left\llcorner\Sigma\right.$, or, in other words, $\mu_{T}=\mu_{T}\llcorner\Sigma$ (see [3, Theorem 4.5]).

A $k$-dimensional simplicial current is a rectifiable current $\llbracket \Sigma \rrbracket$, where $\Sigma \subset \mathbb{R}^{n}$ is a $k$ dimensional simplex (i.e. a convex envelope of $k+1$ points, in particular, a segment if $k=1$ ). Finally, we say that a current $T$ is a polyhedral chain if it can be written as a finite linear combination of simplicial currents supported on simplices with mutually disjoint interiors. Polyhedral chains (and hence rectifiable currents) are a dense subset of flat chains with respect to the flat norm.

Given a rectifiable current $T=\theta \llbracket \Sigma \rrbracket$ and a concave nondecreasing function $g: \mathbb{R}^{+} \rightarrow \mathbb{R}^{+}$ satisfying $g(0)=0$, we define the $g$-mass of $T$ by the formula

$$
\mathbb{M}^{g}(T):=\int_{\Sigma} g(\theta(x)) \mathrm{d} \mathcal{H}^{k}(x) .
$$

In particular, if $g(t):=t^{\alpha}$ for given $\alpha \in[0,1]$, then the above expression defines the $\alpha$-mass of $T$, namely,

$$
\mathbb{M}^{\alpha}(T):=\int_{\Sigma} \theta^{\alpha}(x) \mathrm{d} \mathcal{H}^{k}(x)
$$

The functional $\mathbb{M}^{g}$ (in particular, $\mathbb{M}^{\alpha}$ ) is lower semicontinuous on rectifiable currents with respect to the flat norm convergence (this fact can be proven by a technique used in the proof of Lemma 3.2.14 from [13]). Hence it can be extended to a lower semicontinuous functional defined on all flat chains. In what follows, as is customary,we will write $\mathbb{M}$ instead of $\mathbb{M}^{1}$ (and call it simply mass). 
The following easy consequence of the rectifiability theorem due to White [22, Theorem 8.1] depends on the fact that there exist no nonconstant continuous curve $\theta:[0,1] \rightarrow \mathbb{R}$ having finite $\alpha$-length defined by the formula

$$
\theta \mapsto \int_{0}^{1}\left(\left|\theta^{\prime}(t)\right|+\left|\theta^{\prime}(t)\right|^{\alpha}\right) \mathrm{d} t
$$

when $\alpha \in[0,1)$.

THEOREM 2.1 (White) Let $T$ be a current such that $\mathbb{M}(T)<+\infty$ and $\mathbb{M}^{\alpha}(T)<+\infty$ for some $\alpha \in[0,1)$. Then $T$ is rectifiable.

\section{Subcurrents of flat chains}

We will be frequently using the notion of a subcurrent of a given current as introduced in the definition below.

Definition 3.1 We say that $S$ is a subcurrent of $T$, and write $S \leqslant T$, where $T$ and $S$ are $k$ dimensional currents, whenever

$$
\mathbb{M}(T-S)+\mathbb{M}(S) \leqslant \mathbb{M}(T) .
$$

We now provide a series of remarks concerning the above definition.

REMARK 3.2 Since the inequality

$$
\mathbb{M}(T-S)+\mathbb{M}(S) \geqslant \mathbb{M}(T)
$$

is always true, $S$ is a subcurrent of $T$ if and only if equality holds.

REMARK 3.3 If $S \leqslant T$ and $R \leqslant S$, then $R \leqslant T$. In fact,

$$
\mathbb{M}(T) \geqslant \mathbb{M}(S)+\mathbb{M}(T-S) \geqslant \mathbb{M}(R)+\mathbb{M}(S-R)+\mathbb{M}(T-S) \geqslant \mathbb{M}(R)+\mathbb{M}(T-R),
$$

because of the triangle inequality $\mathbb{M}(T-R) \leqslant \mathbb{M}(T-S)+\mathbb{M}(S-R)$.

REMARK 3.4 Let $T$ be a current with finite mass and let $e \subset \mathbb{R}^{n}$ be a Borel set. Then $T\llcorner e \leqslant T$. In fact,

$$
\mathbb{M}(T)=\mu_{T}\left(\mathbb{R}^{n}\right)=\mu_{T}(e)+\mu_{T}\left(\mathbb{R}^{n} \backslash e\right)=\mathbb{M}(T\llcorner e)+\mathbb{M}(T-T\llcorner e) .
$$

REMARK 3.5 Notice that $S \leqslant T$ in general does not imply

$$
\mathbb{M}^{\alpha}(T)=\mathbb{M}^{\alpha}(T-S)+\mathbb{M}^{\alpha}(S)
$$

when $\alpha \in[0,1)$ (take for example $T \neq 0, S=T / 2$ ). However, if $S=T\llcorner e$ for some Borel set $e \subset \mathbb{R}^{n}$, then the above relationship holds whenever $\mathbb{M}^{\alpha}(T)<+\infty$ and $\mathbb{M}(T)<+\infty$. In fact, in this case, $T$ turns out, in view of Theorem 2.1, to be a rectifiable current $T=\theta \llbracket \Sigma \rrbracket$ for some $\left(\mathcal{H}^{k}, k\right)$-rectifiable set $\Sigma \subset \mathbb{R}^{n}$ and some $\theta \in L^{1}\left(\mathcal{H}^{1}\llcorner\Sigma)\right.$. Then

$$
\mathbb{M}^{\alpha}(T)=\int_{\Sigma}|\theta|^{\alpha} \mathrm{d} \mathcal{H}^{k}=\int_{\Sigma \backslash e}|\theta|^{\alpha} \mathrm{d} \mathcal{H}^{k}+\int_{\Sigma \cap e}|\theta|^{\alpha} \mathrm{d} \mathcal{H}^{k}=\mathbb{M}^{\alpha}(T-S)+\mathbb{M}^{\alpha}(S) .
$$


REMARK 3.6 If $T$ is a current with finite mass and $S \leqslant T$, then for every Borel set $e \subset \mathbb{R}^{n}$ one has $S\llcorner e \leqslant T\llcorner e$. In fact, by the triangle inequality

$$
\begin{aligned}
\mathbb{M}(T\llcorner e) & \leqslant \mathbb{M}((T-S)\llcorner e)+\mathbb{M}(S\llcorner e), \\
\mathbb{M}\left(T\left\llcorner\mathbb{R}^{n} \backslash e\right)\right. & \leqslant \mathbb{M}\left((T-S)\left\llcorner\mathbb{R}^{n} \backslash e\right)+\mathbb{M}\left(S\left\llcorner\mathbb{R}^{n} \backslash e\right),\right.\right.
\end{aligned}
$$

for every Borel $e \subset \mathbb{R}^{n}$, while if we sum the above inequalities, then as a result we get an equality since $S \leqslant T$. Hence the above inequalities are in fact equalities for all Borel $e \subset \mathbb{R}^{n}$. In particular, this also implies

$$
\mu_{T}=\mu_{T-S}+\mu_{S},
$$

and hence $\mu_{S} \leqslant \mu_{T}$. On the other hand, if (5) holds, then $S \leqslant T$ since

$$
\mathbb{M}(S)+\mathbb{M}(T-S)=\mu_{S}\left(\mathbb{R}^{n}\right)+\mu_{T-S}\left(\mathbb{R}^{n}\right)=\mu_{T}\left(\mathbb{R}^{n}\right)=\mathbb{M}(T) .
$$

The following lemma gives an easy characterization of subcurrents of flat chains of finite mass.

LEMmA 3.7 Let a current $T$ have finite mass and assume $S \leqslant T$. Then the representation $T=$ $\tau_{T} \wedge \mu_{T}$ implies $S=\tau_{T} \wedge \sigma \mu_{T}, \mu_{S}=\sigma \mu_{T}$ for some Borel function $\sigma: \mathbb{R}^{n} \rightarrow \mathbb{R}$ satisfying $0 \leqslant \sigma \leqslant 1$ (in other words, $S=T \wedge \sigma$ ). In particular, if $T$ is a rectifiable flat chain, then so is $S$. Further, if $T$ is a flat chain, then $\mathbb{M}^{\alpha}(S) \leqslant \mathbb{M}^{\alpha}(T)$ for all $\alpha \in[0,1]$. If, moreover, $\mathbb{M}(T-S) \neq 0$ and $\mathbb{M}^{\alpha}(T)<+\infty$ for some $\alpha \in[0,1]$, then $\mathbb{M}^{\alpha}(S)<\mathbb{M}^{\alpha}(T)$.

Proof. By Remark 3.6 one has $\mu_{S} \leqslant \mu_{T}$ and hence $\mu_{S}=\sigma \mu_{T}$ for some Borel function $\sigma$ satisfying $0 \leqslant \sigma \leqslant 1$. Since according to the same remark, $\mu_{T-S}=\mu_{T}-\mu_{S}$, we also get $\mu_{T-S}=(1-\sigma) \mu_{T}$. Representing then $S=\tau_{S} \wedge \mu_{S}$ and $T-S=\tau_{T-S} \wedge \mu_{T-S}$, we get

$$
\tau_{T} \wedge \mu_{T}=T=\tau_{S} \wedge \mu_{S}+\tau_{T-S} \wedge \mu_{T-S}=\left(\sigma \tau_{S}+(1-\sigma) \tau_{T-S}\right) \wedge \mu_{T} .
$$

Hence, $\tau_{T}=\sigma \tau_{S}+(1-\sigma) \tau_{T-S}$, and since $\tau_{T}, \tau_{S}$ and $\tau_{T-S}$ are unit vectors, we observe that whenever $\sigma(x)>0$ one has $\tau_{S}(x)=\tau_{T}(x)$. In particular $\sigma \tau_{S}=\sigma \tau_{T}$ and hence $\tau_{S} \wedge \mu_{S}=$ $\tau_{S} \wedge \sigma \mu_{T}=\tau_{T} \wedge \sigma \mu_{T}$. This concludes the proof of the first claim.

If $T$ is a rectifiable flat chain, then $\mu_{T}=\sigma \mathcal{H}^{k}\left\llcorner\Sigma\right.$ for some countably $\left(\mathcal{H}^{k}, k\right)$-rectifiable $\Sigma \subset$ $\mathbb{R}^{n}$ and some $\theta \in L^{1}\left(\Sigma ; \mathcal{H}^{k}\right)$, while $\tau_{T}(x)$ orients the approximate tangent plane to $\Sigma$ at $x$ for $\mathcal{H}^{k}$-a.e. $x \in \Sigma$. One then has $S=\tau_{T} \wedge \sigma \theta \mathcal{H}^{k}\llcorner\Sigma$, which means that $S$ is still rectifiable.

Finally, let $T$ be a flat chain of finite mass. Suppose $\alpha<1$ (otherwise the conclusion follows trivially from the definition of a subcurrent) and $\mathbb{M}^{\alpha}(T)<+\infty$ (otherwise there is nothing to prove). Then by Theorem 2.1. we know that $T$ is rectifiable, i.e. $T=\tau_{T} \wedge \theta \mathcal{H}^{k}\llcorner\Sigma$ with $\Sigma, \theta$ and $\tau_{T}$ as above. Then

$$
\mathbb{M}^{\alpha}(T)=\int_{\Sigma}|\theta|^{\alpha} \mathrm{d} \mathcal{H}^{k}
$$

But then $S=\tau_{T} \wedge \sigma \theta \mathcal{H}^{k}\llcorner\Sigma$ and hence

$$
\mathbb{M}^{\alpha}(S)=\int_{\Sigma}|\sigma \theta|^{\alpha} \mathrm{d} \mathcal{H}^{k} \leqslant \mathbb{M}^{\alpha}(T)
$$

since $|\sigma| \leqslant 1$. Moreover, if $\mathbb{M}^{\alpha}(T)<+\infty$, then $\mathbb{M}^{\alpha}(S)=\mathbb{M}^{\alpha}(T)$ only when $\sigma=1 \mathcal{H}^{k}$-a.e. over $\Sigma$, which means $T=S$ and hence $\mathbb{M}(T-S)=0$. 
REMARK 3.8 If $S \leqslant T$ then $S \leqslant T+S$. In fact, by Lemma 3.7, one has $S=\tau_{T} \wedge \sigma \mu_{T}$ and hence $T+S=\tau_{T} \wedge(1+\sigma) \mu_{T}$ so that $\mu_{T+S}=(1+\sigma) \mu_{T}$, which means that $T \leqslant T+S$ and hence $S \leqslant T+S$.

Lemma 3.9 Let $A$ and $B$ be subcurrents of $T$. Then $A \leqslant A+B$ and consequently $B \leqslant A+B$. If, moreover, $\mu_{A} \wedge \mu_{B}=0$, then $A+B \leqslant T$.

Proof. By Lemma 3.7 we have $A=\tau_{T} \wedge \sigma_{A} \mu_{T}$ and $B=\tau_{T} \wedge \sigma_{B} \mu_{T}$ with $0 \leqslant \sigma_{A} \leqslant 1,0 \leqslant \sigma_{B} \leqslant 1$. Then $A+B=\tau_{T} \wedge\left(\sigma_{A}+\sigma_{B}\right) \mu_{T}$ and hence $\mu_{A+B}=\mu_{A}+\mu_{B}$, which means $A \leqslant A+B$.

If we also suppose $\mu_{A} \wedge \mu_{B}=0$, we will have $\sigma_{A}+\sigma_{B} \leqslant 1$. Hence $\mu_{T-A-B}=\left(1-\sigma_{A}-\sigma_{B}\right) \mu_{T}$. Therefore, $\mu_{A+B}+\mu_{T-A-B}=\mu_{T}$, which means $A+B \leqslant T$.

LEMMA 3.10 Let $\left\{S_{\nu}\right\},\left\{T_{\nu}\right\}$ be sequences of currents with $S_{v} \leqslant T_{\nu}$, and suppose that both $S_{\nu} \rightarrow S$ and $T_{v} \rightarrow T$ weakly as currents as $v \rightarrow \infty$, while $\mathbb{M}\left(T_{v}\right) \rightarrow \mathbb{M}(T)$. Then $\mathbb{M}(T)<+\infty$ implies that $S \leqslant T$ and $\mathbb{M}\left(S_{v}\right) \rightarrow \mathbb{M}(S)$.

Proof. Consider the sequence $\left\{T_{v}-S_{v}\right\}$ which converges to $T-S$ weakly in the sense of currents. By the lower semicontinuity of $\mathbb{M}$ we know that

$$
\begin{aligned}
\mathbb{M}(S)+\mathbb{M}(T-S) & \leqslant \liminf _{k \rightarrow \infty} \mathbb{M}\left(S_{v}\right)+\liminf _{k \rightarrow \infty} \mathbb{M}\left(T_{v}-S_{v}\right) \\
& \leqslant \liminf _{k \rightarrow \infty}\left[\mathbb{M}\left(S_{v}\right)+\mathbb{M}\left(T_{v}-S_{v}\right)\right] \leqslant \liminf _{k \rightarrow \infty} \mathbb{M}\left(T_{v}\right)=\mathbb{M}(T),
\end{aligned}
$$

i.e. $S \leqslant T$. Since we also have $\mathbb{M}(T) \leqslant \mathbb{M}(S)+\mathbb{M}(T-S)$, the inequalities in 6 are actually equalities. Also, since $\mathbb{M}(T-S) \leqslant \liminf _{v} \mathbb{M}\left(T_{v}-S_{v}\right)$ we obtain $\mathbb{M}(S)=\liminf _{v} \mathbb{M}\left(S_{v}\right)$. This is also true for every subsequence of $S_{v}$, hence we have full convergence of the sequence $\mathbb{M}\left(S_{v}\right)$ to $\mathbb{M}(S)$ as $v \rightarrow \infty$.

We now give the following definition which will be crucial in what follows.

Definition 3.11 Let $T$ be a current with $\mathbb{M}(T)<+\infty$. We say that $C$ is a cycle of a current $T$ if $C \leqslant T$ and $\partial C=0$. We say that $T$ is acyclic if $C=0$ is the only cycle of $T$.

We are now able to prove the existence of a "maximum cycle" of every current $T$ with finite mass, i.e. a cycle such that $T-C$ is acyclic.

Proposition 3.12 Every current $T$ with finite mass contains a cycle $C$ such that $T-C$ is acyclic.

Proof. Let

$$
\xi=\sup \{\mathbb{M}(C): C \text { is a cycle of } T\} .
$$

Clearly $\xi<+\infty$ since $\mathbb{M}(C) \leqslant \mathbb{M}(T)$ for every cycle $C$ of $T$. Also $\xi \geqslant 0$ since $C=0$ is always a cycle of $T$.

Step 1. We claim that there exists a cycle $C$ of $T$ such that $\mathbb{M}(C)=\xi$. In fact, by definition of $\xi$, there exists a sequence $\left\{C_{v}\right\}$ of cycles of $T$ such that

$$
\mathbb{M}\left(C_{v}\right) \geqslant \xi-1 / \nu
$$

Clearly $\mathbb{M}\left(C_{v}\right) \leqslant \mathbb{M}(T)$ and $\mathbb{M}\left(\partial C_{v}\right)=0$ so, up to a subsequence, the currents $C_{v}$ converge to a limit $C$ with $\partial C=0$. By Lemma 3.10 (applied with $T_{v}:=T$ ) the current $C$ is itself a cycle of $T$ and $\mathbb{M}(C)=\lim _{v} \mathbb{M}\left(C_{v}\right)=\xi$. 
Step 2. We only have to prove that $T-C$ is acyclic. Let $D$ be any cycle of $T-C$. Since $D \leqslant T-C$ and $C \leqslant T$ we also have $T-C-D \leqslant T-C$ and $T-C \leqslant T$ so we get $(\operatorname{Remark} 3.3) T-C-D \leqslant T$ and $C+D \leqslant T$. Hence we actually have

$$
\mathbb{M}(T)-\mathbb{M}(C)=\mathbb{M}(T-C)=\mathbb{M}(D)+\mathbb{M}(T-C-D)=\mathbb{M}(D)+\mathbb{M}(T)-\mathbb{M}(C+D),
$$

i.e. $\mathbb{M}(C)+\mathbb{M}(D)=\mathbb{M}(C+D)$, which reads $C \leqslant C+D$. Since $C+D$ is a cycle of $T$ we have $\mathbb{M}(C+D) \leqslant \xi$ and as $\mathbb{M}(C)=\xi$ we have $\mathbb{M}(D)=0$, i.e. $D=0$. Since this is true for every cycle $D$ of $T-C$, we conclude that $T-C$ is acyclic.

Finally, the following easy assertion will be used.

LEMMA 3.13 Let $T$ be a polyhedral $k$-dimensional chain and let $S \leqslant T$ be its subcurrent such that $\partial S \leqslant \partial T$. Then $S$ is itself polyhedral.

Proof. One has $T=\sum_{i=1}^{k} \theta_{i} \llbracket \Sigma_{i} \rrbracket$, where $\Sigma_{i} \in \mathbb{R}^{n}$ are pairwise disjoint $k$-simplices and $\theta_{i} \in \mathbb{R}$. Since $S \leqslant T$, by Lemma 3.7 one has $S=T \wedge \sigma$ for some Borel function $\sigma$ satisfying $0 \leqslant \sigma \leqslant 1$. Now $\sigma$ is constant over each simplex $\Sigma_{i}$, since otherwise one would not have $\partial S \leqslant \partial T$. Hence, $S=\sum_{i=1}^{k} \theta_{i} \sigma_{i} \llbracket \Sigma_{i} \rrbracket$, where $\sigma_{i}$ is the value of $\sigma$ over $\Sigma_{i}$, or, in other words, $S$ is still polyhedral.

\section{Concave functionals on flat chains}

The following definition will be crucial.

Definition 4.1 We say that the functional $T \mapsto F(T) \in[0,+\infty]$ defined on $k$-dimensional real flat chains with finite mass is

(i) concave (resp. strictly concave) if the function $f:[-1,+\infty) \rightarrow \mathbb{R}$ defined by

$$
f(t):=F(T+t S)
$$

is concave (resp. strictly concave) whenever $F(T)<+\infty, S \leqslant T, S \neq 0$;

(ii) nondecreasing if

$$
F(S) \leqslant F(T)
$$

whenever $S \leqslant T, S \neq T$. We say that $F$ is strictly increasing if under the same hypotheses we get a strict inequality.

Notice that the above definition of concavity of the functional $F$ can be viewed as the usual concavity of $F$ in the directions given by subcurrents. As an example notice that $\mathbb{M}^{\alpha}$ will be proven to be concave in this sense, but not in the usual sense; in fact one clearly has

$$
\mathbb{M}^{\alpha}\left(\frac{1}{2} T+\frac{1}{2}(-T)\right)=\mathbb{M}^{\alpha}(0)=0<\frac{1}{2} \mathbb{M}^{\alpha}(T)+\frac{1}{2} \mathbb{M}^{\alpha}(-T) \quad \text { for every } T \neq 0
$$

REMARK 4.2 Suppose $F$ is a concave (resp. strictly concave) functional defined on real flat chains of finite mass. If $H:[0,+\infty] \rightarrow[0,+\infty]$ is a concave (resp. strictly concave) function, then the functional $T \mapsto H(F(T))$ is concave (resp. strictly concave). In fact, assume $F(T)<+\infty, S \leqslant T$. If the function $f:[-1,+\infty) \rightarrow \mathbb{R}$ defined by the formula $f(t):=F(T+t S)$ is concave (resp. strictly concave) and $H$ is itself concave (resp. strictly concave) then so is $H \circ f$. 
REMARK 4.3 Clearly, a sum of concave functionals is still concave, and is strictly concave once at least one of the summands is concave.

The following result shows that the functional $\mathbb{M}^{\alpha}$ is concave for $\alpha \in[0,1]$ and strictly concave for $\alpha \in(0,1)$.

Lemma 4.4 Let $\alpha \in[0,1]$, let $T$ be a $k$-dimensional real flat chain satisfying $\mathbb{M}(T)<+\infty$, $\mathbb{M}^{\alpha}(T)<+\infty$ and assume $S \leqslant T$. Consider the function $f:[-1,+\infty) \rightarrow \mathbb{R}$ defined by

$$
f(t):=\mathbb{M}^{\alpha}(T+t S) .
$$

The following properties hold:

(i) $f$ is concave;

(ii) if $S \neq 0$ and $\alpha \in(0,1)$, then $f$ is strictly concave;

(iii) if $\alpha=0$, then $f$ is constant on $(-1,+\infty)$;

(iv) if $\alpha=1$, then $f$ is affine;

(v) if $S=0$, then $f$ is constant.

Proof. By Lemma 3.7 one has $S=\sigma T$ for some Borel function $\sigma$ satisfying $0 \leqslant \sigma \leqslant 1$. For $t \geqslant-1$ one has $1+t \sigma \geqslant 0$ and $\mu_{T+t S}=(1+t \sigma) \mu_{T}$, so that

$$
\mathbb{M}(T+t S)=\mathbb{M}((1+t \sigma) T)=\int_{\mathbb{R}^{n}}|1+t \sigma| \mathrm{d} \mu_{T}=\int_{\mathbb{R}^{n}}(1+t \sigma) \mathrm{d} \mu_{T}=\mathbb{M}(T)+t \mathbb{M}(S),
$$

which proves the claim for $\alpha=1$. If further $\alpha \in[0,1)$ and $\mathbb{M}^{\alpha}(T)<+\infty$, then by Theorem 2.1. $T=\tau_{T} \wedge \theta \mathcal{H}^{k}\left\llcorner\Sigma\right.$ for some countably $\left(\mathcal{H}^{k}, k\right)$-rectifiable $\Sigma \subset \mathbb{R}^{n}$ and some $\theta \in L^{1}\left(\mathcal{H}^{k}\llcorner\Sigma)\right.$, while $\tau_{T}(x)$ orients the approximate tangent plane to $\Sigma$ at $x$ for $\mathcal{H}^{k}$-a.e. $x \in \Sigma$. Then, for $t \geqslant-1$, we get

$$
\mathbb{M}^{\alpha}(T+t S)=\int_{\Sigma}|(1+t \sigma) \theta|^{\alpha} \mathrm{d} \mathcal{H}^{k}=\int_{\Sigma}(1+t \sigma)^{\alpha}|\theta|^{\alpha} \mathrm{d} \mathcal{H}^{k},
$$

which is concave in $t$ for all $\alpha \in(0,1)$, and strictly concave if $S \neq 0$. Finally, for the case $\alpha=0$ we have

$$
f(t)=\mathbb{M}^{0}(T+t S)=\int_{\Sigma} \phi((1+t \sigma) \theta) \mathrm{d} \mathcal{H}^{k}=\int_{\Sigma} \phi(1+t \sigma) \phi(\theta) \mathrm{d} \mathcal{H}^{k},
$$

where

$$
\phi(s):= \begin{cases}0, & s=0 \\ 1, & s>0\end{cases}
$$

and hence

$$
f(t)= \begin{cases}\mathbb{M}^{0}(T-S), & t=-1, \\ \mathbb{M}^{0}(T), & t>-1,\end{cases}
$$

which is constant for $t>-1$ and concave for $t \geqslant-1$.

In the following lemmata, it is convenient to represent the functional $\mathfrak{F}$ in Problem 1 as $\mathfrak{F}(T, S):=$ $F(T)+G(S)$, where

$$
F(T):=A \mathbb{M}^{\alpha}(T), \quad G(S):=B \mathbb{M}^{\beta}(S)+H\left(\mathbb{M}^{\delta}(S)\right) .
$$

We first formulate a very easy auxiliary result regarding concavity of the functionals $F$ and $G$ defined above. 
LEMMA 4.5 The functional $F$ is concave and nondecreasing. It is strictly concave when $A \neq 0$ and $\alpha \in(0,1)$. The functional $G$ is concave and nondecreasing (resp. strictly concave) whenever either $H$ is concave or $\delta=0$ (resp. either $B \neq 0$ and $\beta \in(0,1)$, or $\delta \in(0,1)$ and $H$ is strictly increasing and concave).

Proof. The assertion regarding $F$ follows from Lemma 4.4(i),(ii). By the same lemma the functional $S \mapsto B \mathbb{M}^{\beta}(S)$ is concave (resp. strictly concave when $B \neq 0$ and $\beta \in(0,1)$ ). Further, if $H$ is concave (resp. strictly concave and strictly increasing when $\delta \in(0,1)$ ) we see from Lemma 4.4(i),(ii) and Remark 4.2 (recalling that $H$ is assumed to be nondecreasing) that the functional $S \mapsto H\left(\mathbb{M}^{\delta}(S)\right)$ is concave (resp. strictly concave). Finally, if $\delta=0$, then by Lemma 4.4(iii) the latter functional is constant. Putting all these facts together proves the assertion on $G$.

LEMMA 4.6 Let $F$ and $G$ be two concave, nondecreasing functionals on currents, and let $\mathfrak{F}(T, S):=F(T)+G(S)$. Let $T$ and $S$ be real one-dimensional flat chains of finite mass such that $\mathfrak{F}(T, S)<+\infty$ and either $T$ or $S$ is rectifiable. Then there are two real one-dimensional flat chains $T^{\prime}$ and $S^{\prime}$ of finite mass such that $T^{\prime}+S^{\prime}=T+S, \mu_{T^{\prime}} \wedge \mu_{S^{\prime}}=0, \operatorname{supp}\left(T^{\prime}+S^{\prime}\right) \subset \operatorname{supp} T \cup \operatorname{supp} S$ and $\mathfrak{F}\left(T^{\prime}, S^{\prime}\right) \leqslant \mathfrak{F}(T, S)$.

If, moreover, $T$ and $S$ are not disjoint (in the sense that $\mu_{T} \wedge \mu_{S} \neq 0$ ) and either $F$ or $G$ is strictly concave and strictly increasing, then one has the strict inequality $\mathfrak{F}\left(T^{\prime}, S^{\prime}\right)<\mathfrak{F}(T, S)$.

Proof. Suppose first that $T$ is rectifiable, i.e. $T=\tau_{T} \wedge \theta_{T} \mathcal{H}^{1}\left\llcorner\Sigma_{T}\right.$ (note that $\Sigma_{T} \subset \mathbb{R}^{n}$ is countably $\left(\mathcal{H}^{1}, 1\right)$-rectifiable, while $\tau_{T}(x)$ orients the approximate tangent plane to $\Sigma$ at $x$ for $\mathcal{H}^{1}$-a.e. $\left.x \in \Sigma\right)$. Let $\sigma:=\mu_{T} \wedge \mu_{S}$. If $\sigma \neq 0$ (otherwise one may just take $T^{\prime}:=T, S^{\prime}:=S$ ), then there is a Borel set $\Sigma \subset \Sigma_{T}$ (hence $\Sigma$ is also countably $\left(\mathcal{H}^{1}, 1\right)$-rectifiable) on which $\sigma$ is concentrated. Observe that $\sigma\left(\Sigma \backslash \Sigma_{S}\right)=0$, because

$$
\mu_{S}\left(\Sigma \backslash \Sigma_{S}\right) \leqslant \mu_{S}\left(\Sigma_{T} \backslash \Sigma_{S}\right)=0,
$$

the latter equality being valid in view of the fact that $\mu_{S}\left(E \backslash \Sigma_{S}\right)=0$ for every countably $\left(\mathcal{H}^{1}, 1\right)$ rectifiable set $E \subset \mathbb{R}^{n}$. Hence, we may assume without loss of generality $\Sigma \subset \Sigma_{S}$. We also have $\sigma=\theta \mathcal{H}^{1}\left\llcorner\Sigma\right.$, where $\theta=\theta_{T} \wedge \theta_{S}$.

Set now

$$
\Sigma^{ \pm}:=\left\{x \in \Sigma: \tau_{S}(x)= \pm \tau_{T}(x)\right\} .
$$

Since $S\left\llcorner\Sigma_{S}\right.$ is rectifiable, so is $S\left\llcorner\Sigma\right.$, which implies $\mathcal{H}^{1}\left(\Sigma \backslash\left(\Sigma^{+} \cup \Sigma^{-}\right)\right)=0$. Hence, as $\sigma \ll$ $\mathcal{H}^{1}\llcorner\Sigma$, we get

$$
\sigma\left(\Sigma \backslash\left(\Sigma^{+} \cup \Sigma^{-}\right)\right)=0 .
$$

We first focus our attention on $\Sigma^{-}$and show that one may assume without loss of generality that $\sigma\left(\Sigma^{-}\right)=0$. In fact, if $\sigma\left(\Sigma^{-}\right)>0$, then setting $R:=\tau_{T} \wedge \theta \mathcal{H}^{1}\left\llcorner\Sigma^{-}\right.$, one gets $R \neq 0$, while, clearly, $R \leqslant T$ and $-R \leqslant S$. Set now $\tilde{T}:=T-R, \tilde{S}:=S+R$, and note that $\tilde{T}+\tilde{S}=T+S$. Further, since $\tilde{T} \leqslant T$, we have $F(\tilde{T}) \leqslant F(T)$, and since $\tilde{S} \leqslant S$, we have $G(\tilde{S}) \leqslant G(S)$, and at least one of the above inequalities is strict if either $F$ or $G$ is strictly increasing. Thus we get $\mathfrak{F}(\tilde{T}, \tilde{S}) \leqslant \mathfrak{F}(T, S)$ (with strict inequality if either $F$ or $G$ is strictly increasing). Hence, if one substitutes $\tilde{T}$ for $T$ and $\tilde{S}$ for $S$, one will find that, by construction, $\sigma\left(\Sigma^{-}\right)=0$. Therefore, from now on we assume that $\sigma$ is concentrated on $\Sigma^{+}$, and that $\Sigma=\Sigma^{+}$.

For each $t \in[0,1]$ define

$$
T_{t}:=T+S\left\llcorner\Sigma-t\left(T \left\llcorner\Sigma+S\llcorner\Sigma), \quad S_{t}:=S+T\llcorner\Sigma-(1-t)(T\llcorner\Sigma+S\llcorner\Sigma)\right.\right.\right.
$$


and notice that $T_{t}+S_{t}=T+S$. Also $T\llcorner\Sigma+S\llcorner\Sigma$ is a subcurrent of both $T+S\llcorner\Sigma$ and $S+T\llcorner\Sigma$. Applying Lemma 4.7 (with $T+S\llcorner\Sigma$ instead of $T, S+T\llcorner\Sigma$ instead of $S$ and $T\llcorner\Sigma+S\llcorner\Sigma$ instead of $R$ ), we find that $t \mapsto \mathfrak{F}\left(T_{t}, S_{t}\right)$ is concave (resp. strictly concave if either $F$ or $G$ is). It follows that $t \in[0,1] \mapsto \mathfrak{F}\left(T_{t}, S_{t}\right)$ attains its minimum (resp. strict minimum) at either $\bar{t}=0$ or $\bar{t}=1$. Let $T^{\prime}=T_{\bar{t}}, S^{\prime}=S_{\bar{t}}$. Then $\mathfrak{F}\left(T^{\prime}, S^{\prime}\right) \leqslant \mathfrak{F}(T, S)$ (resp. $\mathfrak{F}\left(T^{\prime}, S^{\prime}\right)<\mathfrak{F}(T, S)$ under either of the conditions (i)-(v) of Lemma 4.7 and when $T\llcorner\Sigma+S\llcorner\Sigma \neq 0$, the latter being true when $\mu_{T} \wedge \mu_{S}>0$ ). To conclude the proof of the statement for rectifiable $T$, we only have to check that $\mu_{T^{\prime}} \wedge \mu_{S^{\prime}}=0$. This is true by construction: in fact, if $\bar{t}=0$, then

$$
T^{\prime}=T+S\left\llcorner\Sigma, \quad S^{\prime}=S-S\llcorner\Sigma\right.
$$

which means that $\mu_{S^{\prime}}$ is concentrated on $\mathbb{R}^{n} \backslash \Sigma$, while $T^{\prime}\left\llcorner\left(\mathbb{R}^{n} \backslash \Sigma\right)=T, S^{\prime}\left\llcorner\left(\mathbb{R}^{n} \backslash \Sigma\right)=S\right.\right.$ and hence

$$
\mu_{T^{\prime}} \wedge \mu_{S^{\prime}} \leqslant\left(\mu_{T^{\prime}\llcorner}\left(\mathbb{R}^{n} \backslash \Sigma\right)\right) \wedge\left(\mu_{S^{\prime}\llcorner}\left(\mathbb{R}^{n} \backslash \Sigma\right)\right)=\left(\mu _ { T } \llcorner ( \mathbb { R } ^ { n } \backslash \Sigma ) ) \wedge \left(\mu_{S}\left\llcorner\left(\mathbb{R}^{n} \backslash \Sigma\right)\right)=0 .\right.\right.
$$

The case $\bar{t}=1$ is completely analogous, since then

$$
T^{\prime}=T-T\left\llcorner\Sigma, \quad S^{\prime}=S+T\llcorner\Sigma,\right.
$$

and hence $\mu_{T^{\prime}}$ is concentrated outside of $\Sigma$, while $T^{\prime}\left\llcorner\left(\mathbb{R}^{n} \backslash \Sigma\right)=T, S^{\prime}\left\llcorner\left(\mathbb{R}^{n} \backslash \Sigma\right)=S\right.\right.$.

The case when $S$ is rectifiable, while $T$ may be arbitrary, is considered in a completely symmetric way.

LEMMA 4.7 Let $F$ and $G$ be two concave functionals defined on real flat chains with finite mass and let $\mathfrak{F}(T, S):=F(T)+G(S)$. Suppose that $T, S, R$ are given real flat chains of finite mass such that $R \leqslant T$ and $R \leqslant S$. Then the function

$$
[0,1] \ni t \mapsto \mathfrak{F}(T-t R, S-(1-t) R)
$$

is concave. Moreover this function is strictly concave if $R \neq 0$ and either $F$ or $G$ is strictly concave. In particular, if $\mathfrak{F}$ is defined as in Problem 1 , then the corresponding function is concave if either $H$ is concave, or $\delta=0$. In this case this function is strictly concave if $R \neq 0$ and either of the following conditions hold:

(i) $\delta=0, \alpha \in(0,1)$ and $A \neq 0$,

(ii) $\delta=0, \beta \in(0,1)$ and $B \neq 0$,

(iii) $H$ is concave, $\alpha \in(0,1)$ and $A \neq 0$,

(iv) $H$ is concave, $\beta \in(0,1)$ and $B \neq 0$,

(v) $H$ is strictly increasing and concave, while $\delta \in(0,1)$.

Proof. From Definition 4.1 we know that both the functions

$$
t \mapsto F(T-t R) \quad \text { and } \quad t \mapsto G(S-(1-t) R)
$$

are concave for $t \in[0,1]$. It suffices to refer now to Remark 4.3 . The case when $\mathfrak{F}$ is as in Problem 1 follows then from Lemma 4.5 . 


\section{Auxiliary lemmata}

We will need the following auxiliary assertions on convergence of measures and currents.

LEMMA 5.1 Let $\phi$ be a signed finite Borel measure with compact support in $\mathbb{R}^{n}$, and $\phi\left(\mathbb{R}^{n}\right)=0$. Then there exists a sequence $\left\{\phi_{\nu}\right\}$ of finite weighted sums of Dirac measures such that

$$
\phi_{v}^{ \pm} \rightarrow \phi^{ \pm}, \quad \phi_{\nu}\left(\mathbb{R}^{n}\right)=0 .
$$

Proof. Consider two sequences $\left\{\psi_{v}^{+}\right\},\left\{\psi_{v}^{-}\right\}$of finite weighted sums of Dirac measures $\psi_{v}^{+} \rightarrow \phi^{+}$ and $\psi_{v}^{-} \rightarrow \phi^{-}$in the $*$-weak sense of measures with $v \rightarrow \infty$ (note that here $\psi_{v}^{+}$and $\psi_{v}^{-}$do not denote the positive and negative parts of some signed measure $\psi_{\nu}$, but just some positive measures; in fact, it may happen that $\psi_{v}^{+} \wedge \psi_{v}^{-} \neq 0$ ).

Consider the quantity $\lambda_{v}:=\psi_{\nu}^{-}\left(\mathbb{R}^{n}\right)-\psi_{\nu}^{+}\left(\mathbb{R}^{n}\right)$ and set

$$
\begin{aligned}
& \tilde{\psi}_{v}^{+}:=\psi_{v}^{+}+\lambda_{v} \delta_{0}, \quad \tilde{\psi}_{v}^{-}:=\psi_{v}^{-} \quad \text { if } \lambda_{v} \geqslant 0, \\
& \tilde{\psi}_{v}^{+}:=\psi_{v}^{+}, \quad \tilde{\psi}_{v}^{-}:=\psi_{v}^{-}-\lambda_{v} \delta_{0} \quad \text { otherwise. }
\end{aligned}
$$

In this way we have $\tilde{\psi}_{v}^{+}\left(\mathbb{R}^{n}\right)=\tilde{\psi}_{v}^{-}\left(\mathbb{R}^{n}\right)$, while both measures $\tilde{\psi}_{v}^{ \pm}$are still nonnegative. Moreover we notice that $\lambda_{v} \rightarrow 0$ because $\psi_{v}^{ \pm}\left(\mathbb{R}^{n}\right) \rightarrow \phi^{ \pm}\left(\mathbb{R}^{n}\right)$ as $v \rightarrow \infty$ and $\phi^{-}\left(\mathbb{R}^{n}\right)-\phi^{+}\left(\mathbb{R}^{n}\right)=\phi\left(\mathbb{R}^{n}\right)=$ 0 . In particular, $\lambda_{v} \delta_{0} \rightarrow 0$ and hence $\tilde{\psi}_{v}^{ \pm} \rightarrow \phi^{ \pm} *$-weakly in the sense of measures as $v \rightarrow \infty$.

We now modify $\tilde{\psi}_{v}^{ \pm}$into $\phi_{v}^{ \pm}$so that $\phi_{v}^{+} \wedge \phi_{v}^{-}=0$. To achieve this result we define $\mu_{v}:=$ $\tilde{\psi}_{v}^{+} \wedge \tilde{\psi}_{v}^{-}$and

$$
\phi_{v}^{ \pm}:=\tilde{\psi}_{v}^{ \pm}-\mu_{v} .
$$

Given any $*$-weakly convergent (in the sense of measures) subsequence of $\left\{\mu_{v}\right\}$, for its limit $\mu$ one has $\mu \leqslant \phi^{ \pm}$because $\mu_{v} \leqslant \tilde{\psi}_{v}^{ \pm}$and $\tilde{\psi}_{v}^{ \pm} \rightarrow \phi^{ \pm} *$-weakly in the sense of measures as $v \rightarrow \infty$. Therefore, since $\phi^{+} \wedge \phi^{-}=0$, one has $\mu=0$. Hence, $\mu_{v} \rightarrow 0$, which implies that $\phi_{v}^{ \pm} \rightarrow \phi^{ \pm}$ $*$-weakly in the sense of measures as $v \rightarrow \infty$. On the other hand, by construction, $\phi_{v}^{+} \wedge \phi_{v}^{-}=0$ and hence the measure $\phi_{v}:=\phi_{v}^{+}-\phi_{v}^{-}$has $\phi_{v}^{ \pm}$as the positive and negative parts. Moreover we easily find that

$$
\phi_{v}\left(\mathbb{R}^{n}\right)=\phi_{v}^{+}\left(\mathbb{R}^{n}\right)-\phi_{v}^{-}\left(\mathbb{R}^{n}\right)=\tilde{\psi}_{v}^{+}\left(\mathbb{R}^{n}\right)-\tilde{\psi}_{v}^{-}\left(\mathbb{R}^{n}\right)=0,
$$

concluding the proof.

LEMMA 5.2 Let $\psi_{v}$ be signed measures on $\mathbb{R}^{n}$ such that $\psi_{v} \rightarrow 0 *$-weakly in the sense of measures as $v \rightarrow \infty$, supp $\psi_{v} \subset K \Subset \mathbb{R}^{n}, \psi_{v}\left(\mathbb{R}^{n}\right)=0$ and $\psi_{v}^{ \pm}\left(\mathbb{R}^{n}\right)<+\infty$. Then there exists a real flat chain $R_{v}$ such that $\partial R_{v}=\psi_{v}$ and $\mathbb{M}\left(R_{v}\right) \rightarrow 0$ as $v \rightarrow \infty$. Moreover, if $\psi_{v}$ is a finite sum of signed Dirac masses, then one may choose $R_{\nu}$ polyhedral.

Proof. Let $R_{\nu}$ provide the minimum of $T \mapsto \mathbb{M}(T)$ among all flat chains $T$ satisfying $\partial T=\psi_{\nu}$. In other words, $R_{v}$ solves the classical Monge-Kantorovich optimal transportation problem of transporting $\psi_{v}^{+}$to $\psi_{v}^{-}$as stated in Subsection 1.1 . Then $\mathbb{M}\left(R_{v}\right)$ is the Wasserstein distance between $\psi_{v}^{+}$to $\psi_{v}^{-}$which metrizes the $*$-weak topology of measures on the set of finite nonnegative Borel measures over the compact $K \subset \mathbb{R}^{n}$. Hence, $\mathbb{M}\left(R_{v}\right) \rightarrow 0$ whenever $\psi_{v} \rightarrow 0 *$-weakly in the sense of measures as $v \rightarrow \infty$. It is also well known that if $\psi_{v}$ is a finite sum of signed Dirac masses, then $R_{v}$ is polyhedral.

LEMMA 5.3 Let $T$ be a one-dimensional real normal current. Then there is a sequence of onedimensional real polyhedral chains $T_{v}$ which converges in the flat norm to $T$, and also $\mathbb{M}\left(T_{\nu}\right) \rightarrow$ $\mathbb{M}(T)$ and $\left(\partial T_{\nu}\right)^{ \pm} \rightarrow(\partial T)^{ \pm} *$-weakly in the sense of measures as $v \rightarrow \infty$. Moreover, if $T$ is acyclic, then one can choose $T_{v}$ to be acyclic too. 
Proof. Let $\left\{\phi_{\nu}\right\}$ be a sequence of finite Borel measures constructed by means of Lemma 5.1 applied to $\phi:=\partial T$. Let also $S_{v}$ be one-dimensional real polyhedral chains satisfying $S_{v} \stackrel{\mathcal{F}}{\rightarrow} T$ and $\mathbb{M}\left(S_{v}\right) \rightarrow$ $\mathbb{M}(T)$ as $v \rightarrow \infty$. By Lemma 5.2 applied to $\psi_{v}:=\phi_{v}-\partial S_{v}$ there is a sequence of one-dimensional real polyhedral chains $R_{v}$ with $\partial R_{v}=\psi_{v}$ and $\mathbb{M}\left(R_{v}\right) \rightarrow 0$. Then the current $T_{v}:=S_{v}+R_{v}$ satisfies the first part of the assertion. In fact, $T_{v} \stackrel{\mathcal{F}}{\rightarrow} T$ as $v \rightarrow \infty$ and $\mathbb{M}\left(T_{v}\right) \leqslant \mathbb{M}\left(S_{v}\right)+\mathbb{M}\left(R_{v}\right)$. Passing to the limit we obtain $\mathbb{M}(T) \leqslant \lim _{v} \mathbb{M}\left(T_{v}\right) \leqslant \lim _{v} \mathbb{M}\left(S_{v}\right)=\mathbb{M}(T)$, and hence $\mathbb{M}\left(T_{v}\right) \rightarrow \mathbb{M}(T)$ as $v \rightarrow \infty$. Also $\left(\partial T_{v}\right)^{ \pm}=\phi^{ \pm} \rightarrow(\partial T)^{ \pm}$as $v \rightarrow \infty$ by construction.

If $T$ is acyclic, we modify $T_{v}$ in the following way. Let $C_{v}$ be the cycle of $T_{v}$ given by Proposition 3.12 such that $T_{v}^{\prime}:=T_{v}-C_{v}$ is acyclic. Up to a subsequence, $C_{v} \stackrel{\mathcal{F}}{\rightarrow} C$ as $v \rightarrow \infty$. Hence, by Lemma 3.10, $\mathbb{M}\left(C_{v}\right) \rightarrow \mathbb{M}(C)$ as $v \rightarrow \infty$ and $C$ is a cycle of $T$. Since the only cycle of $T$ is 0 we conclude that $\mathbb{M}\left(C_{v}\right) \rightarrow 0$, which means that $T_{v}^{\prime} \stackrel{\mathcal{F}}{\rightarrow} T$ and $\mathbb{M}\left(T_{v}^{\prime}\right) \rightarrow \mathbb{M}(T)$ as $v \rightarrow \infty$. It remains to observe that $\partial T_{v}^{\prime}=\partial T_{v}=\phi_{v}$, while by Lemma $3.13, T_{v}^{\prime}$ is still polyhedral.

\section{Currents versus transports}

We call two Lipschitz-continuous curves $\hat{\theta}_{1}, \hat{\theta}_{2}:[0,1] \rightarrow \mathbb{R}^{n}$ equivalent if there is a continuous surjective nondecreasing function (usually called "reparameterization") $\phi:[0,1] \rightarrow[0,1]$ such that $\hat{\theta}_{1}(t)=\hat{\theta}_{2}(\phi(t))$ for all $t \in[0,1]$. Let then $\Theta$ stand for the set of equivalence classes of Lipschitzcontinuous paths. In this way each $\theta \in \Theta$ can be clearly identified with some directed rectifiable curve. We will frequently slightly abuse the language, identifying the elements of $\Theta$ (i.e. directed rectifiable curves) with their parameterizations (i.e. Lipschitz-continuous paths parameterizing such curves), when it cannot lead to a confusion. We consider the set $\Theta$ to be equipped with the distance

$$
d_{\Theta}\left(\theta_{1}, \theta_{2}\right):=\inf \left\{\max _{t \in[0,1]}\left|\hat{\theta}_{1}(t)-\hat{\theta}_{2}(t)\right|: \hat{\theta}_{i} \text { a parameterization of } \theta_{i}, i=1,2\right\},
$$

where $|\cdot|$ is the Euclidean norm in $\mathbb{R}^{n}$. It is easy to see that $\theta_{v} \rightarrow \theta$ in $\Theta$ implies the Hausdorff convergence of the respective traces, though the converse is clearly not true. It is further important to mention that every subset of $\Theta$ made by all paths with uniformly bounded length is clearly compact with respect to the introduced topology. This implies that the whole metric space $\Theta$ is $\sigma$-compact (i.e. a countable union of compact sets).

We will also use the following notions. We say that $\sigma \in \Theta$ is contained in a given $\theta \in \Theta$ if for some parameterizations of $\sigma$ and $\theta$ and for some affine nondecreasing $\phi:[0,1] \rightarrow[0,1]$ one has $\theta(\phi(t))=\sigma(t)$ for all $t \in[0,1]$, which means that $\sigma$ represents a "piece" of $\theta$. Finally, we call $\theta \in \Theta$ an $\operatorname{arc}$ if it is injective.

To each $\theta \in \Theta$ we associate the integral one-dimensional current $\llbracket \theta \rrbracket$ defined by the formula

$$
\llbracket \theta \rrbracket(\omega):=\int_{0}^{1}\langle\dot{\theta}(t), \omega(\theta(t))\rangle \mathrm{d} t
$$

(note that the integral does not depend on the parameterization of $\theta$ so it is well defined on equivalence classes $\theta \in \Theta$ ). We also define the parametric length of $\theta$ as

$$
\ell(\theta):=\int_{0}^{1}|\dot{\theta}(t)| \mathrm{d} t .
$$


Clearly,

$$
\mathbb{M}(\llbracket \theta \rrbracket)=\sup \left\{\llbracket \theta \rrbracket(\omega):\|\omega\|_{\infty} \leqslant 1\right\} \leqslant \ell(\theta) .
$$

The following rather simple assertion is valid.

Lemma 6.1 The map $\theta \in \Theta \mapsto \llbracket \theta \rrbracket$ is a continuous embedding of each subset of curves from $\Theta$ with uniformly bounded lengths into the space of integral one-dimensional currents endowed with the weak topology of currents.

Proof. Let $\theta_{v} \in \Theta$ be curves with uniformly bounded length, i.e. $\ell\left(\theta_{v}\right) \leqslant C<+\infty$ for all $v \in \mathbb{N}$. One has to prove that $\theta_{v} \rightarrow \theta \in \Theta$ as $v \rightarrow \infty$ implies $\llbracket \theta_{\nu} \rrbracket(\omega) \rightarrow \llbracket \theta \rrbracket(\omega)$ for every $\mathrm{C}^{\infty}$ 1 -form $\omega$. Consider the parameterizations of $\theta_{v}$ with $\left|\dot{\theta}_{v}\right| \leqslant C$ for all $t \in[0,1]$. Since $\theta_{v}(t)$, for all $t \in[0,1]$ and $v \in \mathbb{N}$, are contained in some neighbourhood of $\theta$, the sequence $\left\{\theta_{v}\right\}$ is weakly compact in $W^{1,2}\left([0,1] ; \mathbb{R}^{n}\right)$. Hence, up to a subsequence, $\theta_{v} \rightarrow \sigma$ weakly in $W^{1,2}\left([0,1] ; \mathbb{R}^{n}\right)$ as $v \rightarrow \infty$ for some $\sigma \in W^{1,2}\left([0,1] ; \mathbb{R}^{n}\right)$, which in particular means that $\sigma=\theta$, and hence $\dot{\theta}_{v} \rightarrow \dot{\theta}$ weakly in $L^{2}\left([0,1] ; \mathbb{R}^{n}\right)$ as $v \rightarrow \infty$. Hence,

$$
\llbracket \theta_{\nu} \rrbracket(\omega)=\int_{0}^{1}\left\langle\dot{\theta}_{\nu}(t), \omega\left(\theta_{v}(t)\right)\right\rangle \mathrm{d} t \rightarrow \int_{0}^{1}\langle\dot{\theta}(t), \omega(\theta(t))\rangle \mathrm{d} t=\llbracket \theta \rrbracket(\omega)
$$

as $v \rightarrow \infty$.

Given a transport $\eta$ on $\Theta$ we define a functional $T_{\eta}$ on 1-forms as follows:

$$
T_{\eta}(\omega):=\int_{\Theta} \llbracket \theta \rrbracket(\omega) \mathrm{d} \eta(\theta) .
$$

The following theorem shows that $T_{\eta}$ is a normal current under natural assumptions on $\eta$.

THEOREM 6.2 Let $\eta$ be a finite Borel measure on $\Theta$ satisfying

$$
\int_{\Theta} \mathbb{M}(\llbracket \theta \rrbracket) \mathrm{d} \eta(\theta)<+\infty .
$$

Then (8) defines a normal one-dimensional current $T=T_{\eta}$ on $\mathbb{R}^{n}$ with

$$
\partial T=\eta(1)-\eta(0), \quad \text { where } \quad \eta(i):=\left(t_{i}\right)_{\#} \eta, \quad t_{i}(\theta):=\theta(i), \quad i=0,1 .
$$

In particular, if $\eta(1) \wedge \eta(0)=0$, then

$$
(\partial T)^{+}=\eta(1), \quad(\partial T)^{-}=\eta(0) .
$$

Furthermore, for all Borel sets $e \subset \mathbb{R}^{n}$,

$$
\mu_{T}(e) \leqslant \int_{\Theta} \mathbb{M}(\llbracket \theta \rrbracket\llcorner e) \mathrm{d} \eta(\theta) .
$$

Proof. We have to prove that $T=T_{\eta}$ is continuous on $\mathcal{C}^{\infty} 1$-forms, has finite mass and finite boundary mass. According to the definition of mass

$$
\mathbb{M}(T):=\sup \left\{T(\omega):|\omega(x)| \leqslant 1 \text { for all } x \in \mathbb{R}^{n}\right\}
$$


and hence

$$
\mathbb{M}(T) \leqslant \int_{\Theta} \mathbb{M}(\llbracket \theta \rrbracket) \mathrm{d} \eta(\theta)<+\infty
$$

Analogously, the relationships

$$
\begin{aligned}
\mu_{T}(U) & =\sup \left\{T(\omega):|\omega(x)| \leqslant 1, \operatorname{supp} \omega \subset U \text { for all } x \in \mathbb{R}^{n}\right\}, \\
\mathbb{M}(\llbracket \theta \rrbracket\llcorner U) & =\sup \left\{\llbracket \theta \rrbracket(\omega):|\omega(x)| \leqslant 1, \operatorname{supp} \omega \subset U \text { for all } x \in \mathbb{R}^{n}\right\},
\end{aligned}
$$

for every open set $U \subset \mathbb{R}^{n}$ imply

$$
\mu_{T}(e) \leqslant \int_{\Theta} \mathbb{M}(\llbracket \theta \rrbracket\llcorner e) \mathrm{d} \eta(\theta)
$$

for every open set $e \subset \mathbb{R}^{n}$, and hence, for every Borel set $e \subset \mathbb{R}^{n}$. Finally, the computation of the boundary

$$
\begin{aligned}
\partial T(f) & =T(\mathrm{~d} f)=\int_{\Theta}\left(\int_{0}^{1}\langle\nabla f(\theta(t)), \dot{\theta}(t)\rangle \mathrm{d} t\right) \mathrm{d} \eta(\theta) \\
& =\int_{\Theta}\left(\int_{0}^{1} \frac{\mathrm{d}}{\mathrm{d} t} f \circ \theta \mathrm{d} t\right) \mathrm{d} \eta(\theta)=\int_{\Theta}[f(\theta(1))-f(\theta(0))] \mathrm{d} \eta(\theta) \\
& =\int_{\Theta} f\left(t_{1}(\theta)\right) \mathrm{d} \eta(\theta)-\int_{\Theta} f\left(t_{0}(\theta)\right) \mathrm{d} \eta(\theta)=\int_{\mathbb{R}^{n}} f(x) \mathrm{d}(\eta(1)-\eta(0))
\end{aligned}
$$

concludes the proof.

It is worth mentioning that the inequality in 97 may be strict, as the following example shows.

EXAMPLE 1 Let $e_{i}, i=1,2$, stand for the unit vectors along axis $x_{i}$ in $\mathbb{R}^{2}$, and let $\Theta_{1} \subset \Theta$ be a set of paths $\theta$ in $Q:=[0,1] \times[0,1]$ admitting a parameterization $\theta(t)=\left(t, x_{2}\right), t \in[0,1]$, for some $x_{2} \in[0,1]$. Define $\eta_{1}$ by the formula

$$
\eta_{1}(e):=\mathcal{H}^{1}\left(t_{0}\left(e \cap \Theta_{1}\right)\right)
$$

for all Borel $e \subset \Theta$, where $t_{0}(\theta):=\theta(0)$. Clearly, $T_{\eta_{1}}=e_{1} \wedge \mathcal{L}^{2}\left\llcorner Q\right.$. Analogously, letting $\Theta_{2} \subset \Theta$ be a set of paths $\theta$ admitting a parameterization $\theta(t)=\left(x_{1}, t\right), t \in[0,1]$, for some $x_{1} \in[0,1]$, and defining $\eta_{2}$ by the formula

$$
\eta_{2}(e):=\mathcal{H}^{1}\left(t_{0}\left(e \cap \Theta_{2}\right)\right)
$$

for all Borel $e \subset \Theta$, we get $T_{\eta_{2}}=e_{2} \wedge \mathcal{L}^{2}\left\llcorner Q\right.$. Now, setting $\eta:=\eta_{1}+\eta_{2}$, one has $T_{\eta}=T_{\eta_{1}}+T_{\eta_{2}}=$ $\left(e_{1}+e_{2}\right) \wedge \mathcal{L}^{2}\left\llcorner Q\right.$, and hence $\mathbb{M}\left(T_{\eta}\right)=\sqrt{2}$, while

$$
\int_{\Theta} \mathbb{M}(\llbracket \theta \rrbracket) \mathrm{d} \eta=\int_{\Theta_{1}} \mathbb{M}(\llbracket \theta \rrbracket) \mathrm{d} \eta_{1}+\int_{\Theta_{2}} \mathbb{M}(\llbracket \theta \rrbracket) \mathrm{d} \eta_{2}=2>\mathbb{M}\left(T_{\eta}\right) .
$$

We now prove a converse statement, i.e. that given a normal real one-dimensional current $T$, there is a transport $\eta$ satisfying $T=T_{\eta}$. 
THEOREM 6.3 Given a one-dimensional acyclic real normal current $T$ with compact support, there exists a Borel measure $\eta$ over $\Theta$ such that $T=T_{\eta}$ as defined by $(8)$ and

$$
\mathbb{M}(T)=\int_{\Theta} \mathbb{M}(\llbracket \theta \rrbracket) \mathrm{d} \eta(\theta) .
$$

Moreover, one can choose $\eta$ so that

$$
\eta(1)=(\partial T)^{+}, \quad \eta(0)=(\partial T)^{-},
$$

where $(\partial T)^{ \pm}$are the positive and negative parts of the measure $\partial T$ respectively, while $\eta$-a.e. $\theta \in \Theta$ is an arc.

REMARK 6.4 In view of Theorem 6.2, the claim (10) is equivalent to a formally weaker one,

$$
\mathbb{M}(T) \geqslant \int_{\Theta} \mathbb{M}(\llbracket \theta \rrbracket) \mathrm{d} \eta(\theta) .
$$

Apart from the claim (11), which is indeed used in what follows, the above theorem is in fact contained (though in quite different terminology) in Theorem $C$ from [19]. Since the relationship between one-dimensional flat chains of finite mass and transports is of utmost importance in this paper, we provide a complete and independent proof of this result.

Before proving the above Theorem 6.3 in the general case, we need to prove a similar assertion valid only for one-dimensional real polyhedral chains.

LEMma 6.5 Let $T$ be a one-dimensional real polyhedral chain. Then there exists a Borel measure $\eta$ over $\Theta$ such that $T=T_{\eta}$ and

$$
\mathbb{M}(T)=\int_{\Theta} \mathbb{M}(\llbracket \theta \rrbracket) \mathrm{d} \eta(\theta)=\int_{\Theta} \ell(\theta) \mathrm{d} \eta(\theta)
$$

and $\eta$-a.e. $\theta$ is supported on supp $T$. Further, if $T$ is also acyclic, then one can choose $\eta$ so that

$$
\eta(1)=(\partial T)^{+}, \quad \eta(0)=(\partial T)^{-} .
$$

If one does not require (13), one can choose $\eta$ so as to have $\mathcal{H}^{1}(\theta) \leqslant \operatorname{diam} \operatorname{supp} T$ for $\eta$-a.e. $\theta \in \Theta$.

Proof. Every one-dimensional real polyhedral chain $T$ can be written as a finite sum

$$
T=\sum_{\nu} \theta_{\nu} T_{\nu}
$$

where $\theta_{v}>0$ are real multiplicities, and $T_{v}$ are currents associated to oriented segments $T_{v}=$ $\llbracket a_{v}, b_{v} \rrbracket$ (further called edges of $T$ ) with nonoverlapping interiors.

Step 1. If $T$ is a generic one-dimensional real polyhedral chain, consider the Lipschitz curves $\sigma_{v}$ defined by $\sigma_{v}(t):=(1-t) a_{v}+t b_{v}$ for all $t \in[0,1]$, and set

$$
\eta:=\sum_{v} \theta_{v} \delta_{\sigma_{v}}
$$


where $\delta_{\sigma_{v}}$ is the Dirac measure concentrated on $\sigma_{v} \in \Theta$. Clearly,

$$
T(\omega)=\sum_{\nu} \int_{\sigma_{v}} \theta_{\nu} \omega \cdot\left(b_{v}-a_{\nu}\right)=\sum_{\nu} \theta_{\nu} \llbracket \sigma_{\nu} \rrbracket(\omega)=\int_{\Theta} \llbracket \sigma \rrbracket(\omega) \mathrm{d} \eta(\sigma),
$$

i.e. $T=T_{\eta}$. By construction one also has $\mathbb{M}(\llbracket \sigma \rrbracket)=\ell(\sigma)$ for $\eta$-a.e. $\sigma \in \Theta$, and hence

$$
\mathbb{M}(T)=\sum_{\nu}\left|\theta_{v}\right| \cdot\left|b_{v}-a_{v}\right|=\int_{\Theta} \ell(\sigma) \mathrm{d} \eta(\sigma),
$$

while $\eta$-a.e. $\theta \subset \Theta$ is a segment, $\theta \subset \operatorname{supp} T$, and hence $\mathcal{H}^{1}(\theta) \leqslant \operatorname{diam} \operatorname{supp} T$.

Step 2. To consider the case of an acyclic $T$, we introduce some extra notation. We say that an ordered finite collection $\left(T_{v_{1}}, \ldots, T_{\nu_{N}}\right)$ of edges, where $T_{v_{i}}:=\llbracket a_{v_{i}}, b_{v_{i}} \rrbracket, i=1, \ldots, N$, is a path in $T$ if $b_{v_{i}}=a_{v_{i+1}}$ for $i=1, \ldots, N-1$. We say that such a path is closed if also $b_{v_{N}}=a_{v_{1}}$. Choosing $\theta_{0}>0$ to be the minimum of $\theta_{v}$ over all $v$, we notice that the current

$$
\sum_{i=1}^{N} \theta_{0} T_{v_{i}}
$$

is a subcurrent of $T$. An acyclic $T$ therefore contains no closed paths. Finally, given a path in $T$, we can extend it forward if there exists an edge $T_{v}$ of $T$ such that $a_{v}=b_{v_{N}}$, and backward if there exists an edge $T_{v}$ such that $b_{v}=a_{v_{1}}$.

Let $T$ be acyclic. We consider a path with a single edge $T_{\bar{v}}$ such that $\theta_{\bar{v}}=\theta_{0}$. We extend this path as much as possible forward and backward. At each extension step the path is not closed, hence the path is composed by different edges. Since there are only a finite number of edges in $T$, this extension process must terminate in a finite number of steps. We obtain in this way a maximal path containing $T_{\bar{\nu}}$. Let $\left(T_{\nu_{1}}, \ldots, T_{\nu_{N}}\right)$ be this maximal path and consider the corresponding current

$$
P_{0}:=\sum_{i=1}^{N} \theta_{0} T_{v_{i}}
$$

Clearly, $P_{0}$ is a subcurrent of $T$ and $\partial P_{0}=\llbracket b_{v_{N}} \rrbracket-\llbracket a_{v_{1}} \rrbracket$. Since this path is maximal, there is no edge $T_{v}$ with endpoint $b_{v}=a_{v_{1}}$, and thus $\llbracket a_{v_{1}} \rrbracket$ is a subcurrent of $(\partial T)^{-}$. Analogously $\llbracket b_{v_{N}} \rrbracket$ is a subcurrent of $(\partial T)^{+}$. One has

$$
P_{0}(\omega)=\int_{\Theta} \llbracket \sigma \rrbracket(\omega) \mathrm{d} \eta_{0}(\sigma),
$$

where $\eta_{0}:=\theta_{0} \delta_{\sigma_{0}}$ is the Dirac measure with mass $\theta_{0}$ concentrated on the curve $\sigma_{0} \in \Theta$, representing the polygonal line $\left[a_{1}, b_{1}\right] \circ \cdots \circ\left[a_{N}, b_{N}\right]$ (starting at $a_{1}$ and ending at $b_{N}$ ). Hence $\eta_{0}(1)=\left(\partial P_{0}\right)^{+}$and $\eta_{0}(0)=\left(\partial P_{0}\right)^{-}$.

The current $T^{\prime}=T-P_{0}$ is itself a polyhedral acyclic current with strictly fewer edges than $T$ has, because the edge $T_{\bar{v}}$ is not included in $T^{\prime}$. Repeating the previous construction with $T^{\prime}$ in place of $T$ we find a subcurrent $P_{1}$ representing a path in $T^{\prime}$ and such that

$$
P_{1}(\omega)=\int_{\Theta} \llbracket \sigma \rrbracket(\omega) \mathrm{d} \eta_{1}(\sigma)
$$

with $\eta_{1}(1)=\left(\partial P_{1}\right)^{+}$and $\eta_{1}(0)=\left(\partial P_{1}\right)^{-}$. A finite number of such steps will clearly exhaust $T$ and yield a decomposition $T=\sum_{i=0}^{k} P_{i}$ such that the corresponding measure $\eta:=\sum_{i=0}^{k} P_{i}$ has the required properties. 
We are now able to prove the general Theorem 6.3 .

Proof of Theorem 6.3 We divide the proof into two steps.

Step 1. Given an arbitrary one-dimensional real flat chain $T$, we consider a sequence $\left\{T_{\nu}\right\}$ of onedimensional polyhedral chains which converges to $T$ in the flat norm and $\mathbb{M}\left(T_{v}\right) \rightarrow \mathbb{M}(T)$ as $v \rightarrow \infty$, hence in particular $\mathbb{M}\left(T_{v}\right) \leqslant \mathbb{M}(T)+1$ for all sufficiently large $v \in \mathbb{N}$. By Lemma 6.5, for each $T_{v}$ we find a transport $\eta_{v}$ satisfying

$$
T_{\nu}(\omega)=\int_{\Theta} \llbracket \theta \rrbracket(\omega) \mathrm{d} \eta_{\nu}(\theta), \quad \mathbb{M}(T)=\int_{\Theta} \mathbb{M}(\llbracket \theta \rrbracket) \mathrm{d} \eta_{\nu}(\theta)=\int_{\Theta} \ell(\theta) \mathrm{d} \eta_{\nu}(\theta)
$$

for all $\mathrm{e}^{\infty} 1$-forms $\omega$. Since $T$ is acyclic, we choose $T_{v}$ according to Lemma 5.3 i.e. so that in addition $\left(\partial T_{\nu}\right)^{ \pm} \rightarrow(\partial T)^{ \pm}$in the $*$-weak sense of measures when $v \rightarrow \infty$. In this case by Lemma 6.5 one can choose a transport $\eta_{v}$ satisfying additionally

$$
\eta_{v}(1)=\left(\partial T_{v}\right)^{+}, \quad \eta_{v}(0)=\left(\partial T_{\nu}\right)^{-} .
$$

In particular, the total masses $\eta_{\nu}(\Theta)$ are uniformly bounded.

In view of $(16)$ we have the estimate

$$
\int_{\Theta} \ell(\theta) \mathrm{d} \eta_{\nu}=\mathbb{M}\left(T_{\nu}\right) \leqslant \mathbb{M}(T)+1
$$

Further, without loss of generality we may assume that the traces of $\eta_{v}$-a.e. $\theta \in \Theta$ are supported on some compact $\Omega \subset \mathbb{R}^{n}$. We may therefore invoke Lemma 6.7 below, showing that up to a subsequence, $\eta_{v} \rightarrow \eta$ in the narrow sense of measures for some finite Borel measure $\eta$, and moreover, that one may let $v \rightarrow \infty$ on both sides of the first relationship of (16) obtaining $T(\omega)=T_{\eta}(\omega)$ for each $\complement^{\infty} 1$-form $\omega$, and hence $T=T_{\eta}$. One shows in addition that (11) is valid by passing to the limit as $v \rightarrow \infty$ on both sides of (17).

Furthermore, note that

$$
\mathbb{M}\left(T_{\nu}\right)=\int_{\Theta} \mathbb{M}(\llbracket \theta \rrbracket) \mathrm{d} \eta_{\nu}(\theta)
$$

by the second relationship of $(16)$. Hence, as the functional $\theta \in \Theta \mapsto \mathbb{M}(\llbracket \theta \rrbracket)$ is 1.s.c., and hence the integral in (18) is 1.s.c. with respect to the narrow convergence of $\eta_{\nu}$, by passing to the limit on both sides of (18) as $v \rightarrow \infty$, we deduce

$$
\mathbb{M}(T)=\lim _{\nu} \mathbb{M}\left(T_{\nu}\right)=\lim _{\nu} \int_{\Theta} \mathbb{M}(\llbracket \theta \rrbracket) \mathrm{d} \eta_{\nu}(\theta) \geqslant \int_{\Theta} \mathbb{M}(\llbracket \theta \rrbracket) \mathrm{d} \eta(\theta),
$$

which provides 10 once one recalls Remark 6.4

We also consider for further use the functional $M^{\prime}: \Theta \rightarrow \mathbb{R}^{+}$defined by

$$
M^{\prime}(\eta):=\int_{\Theta} \ell(\theta) \mathrm{d} \eta
$$

It is 1.s.c. with respect to the narrow convergence of measures (because the parametric length $\ell(\cdot)$ is 1.s.c. in $\Theta)$. Hence, noting that $M^{\prime}\left(\eta_{v}\right)=\mathbb{M}\left(T_{v}\right)$ for each $\eta_{v}$ by construction, we get $M^{\prime}(\eta) \leqslant$ $\mathbb{M}(T)<+\infty$. 
Step 2. Finally, for $T$ acyclic, we consider an $\eta$ minimizing $M^{\prime}$ over the set $E$ of all transports $\eta^{\prime}$ satisfying $T=T_{\eta^{\prime}}$, as well as $(10)$ and (11). To prove the existence of such an $\eta$ recall that the latter set of transports is nonempty in view of Step 1. Consider now a minimizing sequence $\left\{\eta_{\nu}\right\} \subset E$ for $M^{\prime}$. By the final remark of Step 1 one has $M^{\prime}\left(\eta_{v}\right) \leqslant C<+\infty$ for some $C>0$. Further, without loss of generality we may assume that the traces of $\eta_{v}$-a.e. $\theta \in \Theta$ are supported on some compact $\Omega \subset \mathbb{R}^{n}$. Hence by Lemma 6.7, the sequence $\left\{\eta_{\nu}\right\}$ admits a subsequence (as usual, not relabelled) converging to some transport $\eta$ in the narrow sense of measures, while $T=T_{\eta_{v}} \rightarrow T_{\eta}$ in the weak sense of currents as $v \rightarrow \infty$, and thus $T=T_{\eta}$. Since by the same lemma $\eta_{v}(i) \rightarrow \eta(i), i=0,1$, in the narrow sense of measures, also (11) holds for $\eta$, while acting as in Step 1, we get the validity of 10) for $\eta$. Summing up, we get $\eta \in E$. Recalling that $M^{\prime}$ is l.s.c. with respect to the narrow convergence of measures, we infer that $\eta$ is a minimizer of $M^{\prime}$ over $E$.

Let $f: \Theta \rightarrow \Theta$ and $g: \Theta \rightarrow \Theta$ be given by Lemma 6.6 below. Then $T_{f_{\sharp} \eta}$ is a cycle of $T=T_{\eta}$. Hence, $T_{f_{\sharp} \eta}=0$. This means $\llbracket f(\theta) \rrbracket=0$ for $\eta$-a.e. $\theta \in \Theta$. We thus have $\llbracket g(\theta) \rrbracket=\llbracket \theta \rrbracket$ for $\eta$-a.e. $\theta \in \Theta$. Hence, $T_{g_{\#} \eta}=T_{\eta}=T$ and $g_{\#} \eta \in E$, so

$$
M^{\prime}\left(g_{\#} \eta\right)=\int_{\Theta} \ell(g(\theta)) \mathrm{d} \eta(\theta) \leqslant \int_{\Theta} \ell(\theta) \mathrm{d} \eta(\theta) .
$$

Therefore, by the minimality of $\eta$ for $M^{\prime}$, we get $\ell(g(\theta))=\ell(\theta)$, hence $\eta$-a.e. $\theta \in \Theta$ is an arc, which concludes the proof.

The following technical assertions have been used in the proof of Theorem 6.3

LEMma 6.6 (i) There is a map $f: \Theta \rightarrow \Theta$ measurable with respect to all transports such that $f(\theta)$ is a loop (i.e. a simple closed curve) contained in $\theta \in \Theta$ such that

$$
\ell(f(\theta)) \geqslant \frac{1}{2} \sup \{\ell(\sigma): \sigma \text { is a loop contained in } \theta\} .
$$

(ii) There is a map $g: \Theta \rightarrow \Theta$ measurable with respect to all transports such that for all $\theta \in \Theta$ one has $\theta=g(\theta) \cup f(\theta)$ (as traces), $\llbracket \theta \rrbracket=\llbracket g(\theta) \rrbracket+\llbracket f(\theta) \rrbracket$, while

$$
\ell(g(\theta))<\ell(\theta)
$$

unless $\theta$ is an arc, and finally, $g(\theta)=\theta$ if and only if $\theta$ is an arc.

Proof. We construct a map $f: \Theta \rightarrow \Theta$ satisfying claim (i) as follows. For every $\theta \in \Theta$ and $x \in \theta$ we let $C(\theta, x)$ stand for the set of curves contained in $\theta$ starting and ending at $x$ in the sense that

$$
\begin{aligned}
C(\theta, x)=\{\tilde{\theta} \in \Theta: & \tilde{\theta}(t)=\theta\left((1-t) s_{1}+t s_{2}\right) \\
& \text { for some } \left.0 \leqslant s_{1} \leqslant s_{2} \leqslant 1, \theta\left(s_{1}\right)=\theta\left(s_{2}\right)=x\right\} .
\end{aligned}
$$

In case $x \notin \theta$ we define $C(\theta, x)$ to consist of just a single curve $\theta_{x}$ given by $\theta_{x}(t):=x$ for all $t \in[0,1]$, i.e. of a constant curve whose trace reduces to the single point $x$. Note that $\theta_{x} \in C(\theta, x)$ for all $x \in \mathbb{R}^{n}$. Defined in this way, the multivalued map

$$
(\theta, x) \in \Theta \times \mathbb{R}^{n} \mapsto C(\theta, x) \subset \Theta
$$

is u.s.c. (as a multivalued map), and hence Borel measurable. Therefore, recalling that $\ell: \Theta \rightarrow \mathbb{R}$ is 1.s.c. one gets the Borel measurability of the single-valued map

$$
\lambda: \theta \in \Theta \mapsto \sup _{x \in \mathbb{R}^{n}} \sup \{\ell(\sigma): \sigma \in C(\theta, x)\} \in \mathbb{R} .
$$


Clearly, $\lambda(\theta)$ is the supremum of the lengths of the loops contained in $\theta$. Finally, we define

$$
F: \theta \in \Theta \mapsto\left\{\sigma \in \bigcup_{x \in \theta} C(\theta, x): \ell(\sigma) \geqslant \lambda(\theta) / 2\right\} \subset \Theta .
$$

By the von Neumann-Aumann measurable selection theorem ([12, Theorems III.22 and III.23] or [20, Corollary 5.5.8]) one can find a selection $f: \Theta \rightarrow \Theta$ of the multivalued map $F$ which is measurable with respect to all transports $\eta$. Clearly, $f(\theta)$ is as required.

Define now $g: \Theta \rightarrow \Theta$ as a union of two curvilinear segments, by setting

$$
g(\theta):=[\theta(0), f(\theta)(0)] \circ[f(\theta)(1), \theta(1)] .
$$

Clearly, $g(\theta)$ is obtained by "cancelling" the loop $f(\theta)$ from $\theta$. The properties of $g$ announced in claim (ii) follow immediately since $\ell(g(\theta)) \leqslant \ell(\theta)-\lambda(\theta) / 2$, while $g(\theta)=\theta$ if and only if $f(\theta)=\theta_{x}$ for some $x \in \theta$, i.e. when $\theta$ is an arc.

LEMMA 6.7 Let $\left\{\eta_{\nu}\right\}$ be a sequence of nonnegative finite Borel measures over $\Theta$ with uniformly bounded total masses, and set $T_{\nu}:=T_{\eta_{\nu}}$. Assume that for some one-dimensional real flat chain $T$ with $\mathbb{M}(T)<+\infty$ one has $T_{v} \rightarrow T$ weakly in the sense of currents, $\mathbb{M}\left(T_{v}\right) \rightarrow \mathbb{M}(T)$ as $v \rightarrow \infty$, and

$$
M^{\prime}\left(\eta_{v}\right):=\int_{\Theta} \ell(\theta) \mathrm{d} \eta_{v} \leqslant C<+\infty \quad \text { for all } v \in \mathbb{N},
$$

and there is a compact $\Omega \subset \mathbb{R}^{n}$ such that for each $v \in \mathbb{N}$, the traces of $\eta_{v}$-a.e. $\theta \in \Theta$ are supported in $\Omega$. Then there exists a transport $\eta$ such that up to a subsequence, $\eta_{v} \rightarrow \eta$ (and in particular, $\left.\eta_{\nu}(i) \rightarrow \eta(i), i=0,1\right)$ in the narrow sense of measures. Further, $T=T_{\eta}$ if either of the following two conditions hold:

(i) all $\eta_{v}$ are concentrated on some compact subset of $\Theta$ (independent of $v$ ), or

(ii) $T$ is acyclic and

$$
\mathbb{M}\left(T_{\nu}\right)=\int_{\Theta} \mathbb{M}(\llbracket \theta \rrbracket) \mathrm{d} \eta_{v}(\theta) \quad \text { for all } v \in \mathbb{N} .
$$

Proof. Since for every $c>0$ one has

$$
M^{\prime}\left(\eta_{v}\right)=\int_{\Theta} \ell(\theta) \mathrm{d} \eta_{v} \geqslant c \eta_{v}(\{\ell(\theta)>c\})
$$

we conclude

$$
\eta_{v}(\{\ell(\theta)>c\}) \leqslant C / c .
$$

Recalling now that $\{\theta \in \Theta: \ell(\theta) \leqslant c, \theta \subset \Omega\}$ is a compact subset of $\Theta$, we see that the sequence $\eta_{\nu}$ is tight in the sense of measures. Hence, up to a subsequence, $\eta_{\nu} \rightarrow \eta$ as $v \rightarrow \infty$ in the narrow sense of measures for some finite Borel measure $\eta$ over $\Theta$. The convergence $\eta_{\nu}(i) \rightarrow \eta(i), i=0,1$, as $v \rightarrow \infty$ follows from the fact that a push-forward operator by means of a continuous function is continuous with respect to the narrow convergence of measures.

In the case when (i) holds, i.e. all $\eta_{v}$ are concentrated on some compact subset of $\Theta$, one immediately gets

$$
T_{\nu}(\omega)=\int_{\Theta} \llbracket \theta \rrbracket(\omega) \mathrm{d} \eta_{\nu}(\theta) \rightarrow \int_{\Theta} \llbracket \theta \rrbracket(\omega) \mathrm{d} \eta(\theta)=T_{\eta}(\omega) \quad \text { as } v \rightarrow \infty,
$$

and hence $T=T_{\eta}$. 
Consider now the case when (ii) holds, and in particular, $\eta_{v}$ are not necessarily concentrated on some (unique) compact subset of $\Theta$. We show first that

$$
\phi(k):=\limsup _{\nu} \int_{\{\ell(\theta)>k\}} \ell(\theta) \mathrm{d} \eta_{\nu}(\theta) \rightarrow 0 \quad \text { as } k \rightarrow \infty .
$$

In fact, otherwise there exists a $c>0$ such that for a subsequence of $\eta_{v}$ (not relabelled) one has

$$
\int_{\{\ell(\theta)>v\}} \ell(\theta) \mathrm{d} \eta_{v}(\theta) \geqslant c .
$$

Consider then $\eta_{v}^{\prime}:=\eta_{v}\left\llcorner\{\ell(\theta)>v\}\right.$, and $S_{v}:=T_{\eta_{v}^{\prime}}$. By Remark 6.9 below, each $S_{v}$ is a subcurrent of $T_{v}$, and hence by Lemma 3.10 up to a subsequence, $S_{v} \rightarrow S$ weakly in the sense of currents as $v \rightarrow \infty$, where $S$ is a subcurrent of $T$ and $\mathbb{M}(S) \geqslant c$. On the other hand, since $\eta_{v}^{\prime} \rightarrow 0$,

$$
\partial S_{v}=\eta_{v}^{\prime}(1)-\eta_{v}^{\prime}(0) \rightarrow 0
$$

weakly in the sense of measures as $v \rightarrow \infty$, hence $\partial S=0$ and, by acyclicity of $T$, one gets $S=0$, giving a contradiction. Hence, the claim $(20)$ is proven.

Fix now an arbitrary regular 1-form $\omega$, and for each $\theta \in \Theta, k \in \mathbb{N}$ set

$$
f_{k}(\theta):= \begin{cases}\llbracket \theta \rrbracket(\omega), & \ell(\theta) \leqslant k, \\ 0, & \text { otherwise. }\end{cases}
$$

One gets

$$
\begin{aligned}
\left|\int_{\Theta} \llbracket \theta \rrbracket(\omega) \mathrm{d} \eta_{\nu}(\theta)-\int_{\Theta} f_{k}(\theta) \mathrm{d} \eta_{v}(\theta)\right| & =\left|\int_{\{\ell(\theta)>k\}} \llbracket \theta \rrbracket(\omega) \mathrm{d} \eta_{v}(\theta)\right| \\
& \leqslant\|\omega\|_{\infty} \int_{\{\ell(\theta)>k\}} \mathbb{M}(\llbracket \theta \rrbracket) \mathrm{d} \eta_{\nu}(\theta) \\
& \leqslant\|\omega\|_{\infty} \int_{\{\ell(\theta)>k\}} \ell(\theta) \mathrm{d} \eta_{\nu}(\theta) \\
& =\|\omega\|_{\infty} \phi(k) .
\end{aligned}
$$

Since for each fixed $k$, by Lemma 6.1 .

$$
\int_{\Theta} f_{k}(\theta) \mathrm{d} \eta_{v}(\theta) \rightarrow \int_{\Theta} f_{k}(\theta) \mathrm{d} \eta(\theta) \quad \text { as } v \rightarrow \infty,
$$

we arrive at the estimate

$$
\begin{aligned}
\int_{\Theta} f_{k}(\theta) \mathrm{d} \eta(\theta)-\|\omega\|_{\infty} \phi(k) & \leqslant \liminf _{\nu} \int_{\Theta} \llbracket \theta \rrbracket(\omega) \mathrm{d} \eta_{\nu}(\theta) \leqslant \limsup _{\nu} \int_{\Theta} \llbracket \theta \rrbracket(\omega) \mathrm{d} \eta_{v}(\theta) \\
& \leqslant \int_{\Theta} f_{k}(\theta) \mathrm{d} \eta(\theta)+\|\omega\|_{\infty} \phi(k) .
\end{aligned}
$$

Letting $k \rightarrow \infty$ in the above estimate and taking into account $[20$, we get

$$
T_{\nu}(\omega) \rightarrow \sup _{k} \int_{\Theta} f_{k}(\theta) \mathrm{d} \eta(\theta)=\int_{\Theta} \llbracket \theta \rrbracket(\omega) \mathrm{d} \eta(\theta)=T_{\eta}(\omega) \quad \text { as } v \rightarrow \infty,
$$

which yields $T=T_{\eta}$. 
It is worth remarking that the requirement of acyclicity of the "limit current" $T$ in (ii) of the above Lemma 6.7 is essential, as shown in the example below.

EXAMPLE 2 Consider the sequence of curves in $\mathbb{R}^{2}$ admitting the parameterization $\theta_{\nu}(t):=$ $(1+t / v)(\cos (2 \pi v t), \sin (2 \pi v t)), t \in[0,1]$, and define $\eta_{v}:=(1 / v) \delta_{\theta_{v}}$ be the transport concentrated on $\theta_{v} \in \Theta$ and having total mass $1 / \nu$. Define also $\bar{\theta}(t):=(\cos (2 \pi t), \sin (2 \pi t))$ and let $\eta:=\delta_{\bar{\theta}}$ be the transport concentrated on $\bar{\theta}$ with unit total mass. Clearly $\eta_{v} \rightarrow 0$ in the narrow sense of measures as $v \rightarrow \infty$ (in fact, $\eta_{v}(\Theta)=1 / v$ ). On the other hand, $T_{\eta_{v}} \stackrel{\mathcal{F}}{\rightarrow} T_{\eta} \neq 0$ as $v \rightarrow \infty$. However, this does not contradict Lemma 6.7 because clearly $\partial T_{\eta}=0$, i.e. $T_{\eta}$ is a cycle.

We now concentrate our attention on the restriction to a given Borel set of the currents of the form $T=T_{\eta}$.

Proposition 6.8 Let $T$ be a normal one-dimensional current and $\eta$ be a transport such that $T=$ $T_{\eta}$ and

$$
\mathbb{M}(T)=\int_{\Theta} \mathbb{M}(\llbracket \theta \rrbracket) \mathrm{d} \eta(\theta)
$$

Then $\mu_{T}=\mu_{\llbracket \theta \rrbracket} \otimes \eta$, i.e.

$$
\mu_{T}(e)=\int_{\Theta} \mathbb{M}(\llbracket \theta \rrbracket\llcorner e) \mathrm{d} \eta(\theta)
$$

and moreover,

$$
T\left\llcorner e(\omega)=\int_{\Theta} \llbracket \theta \rrbracket\llcorner e(\omega) \mathrm{d} \eta(\theta),\right.
$$

for every Borel set $e \subset \mathbb{R}^{n}$, where

$$
\llbracket \theta \rrbracket\left\llcorner e(\omega)=\int_{\theta^{-1}(e)}\langle\dot{\theta}(t), \omega(\theta(t))\rangle \mathrm{d} t\right.
$$

(note that the latter integral is independent of the choice of a parameterization of $\theta$ ).

REMARK 6.9 The relationship (21) also implies that for every Borel $e \subset \Theta$ the current $S:=T_{\eta\llcorner e}$ is a subcurrent of $T$. In fact, in this case $T-S=T_{\eta\left\llcorner e^{c}\right.}$, where $e^{c}:=\Theta \backslash e$, and thus, by Theorem 6.2.

$$
\mathbb{M}(S) \leqslant \int_{\Theta} \mathbb{M}(\llbracket \theta \rrbracket) \mathrm{d} \eta\left\llcorner e(\theta), \quad \mathbb{M}(T-S) \leqslant \int_{\Theta} \mathbb{M}(\llbracket \theta \rrbracket) \mathrm{d} \eta\left\llcorner e^{c}(\theta) .\right.\right.
$$

Hence, summing the above inequalities, one gets

$$
\mathbb{M}(S)+\mathbb{M}(T-S) \leqslant \int_{\Theta} \mathbb{M}(\llbracket \theta \rrbracket) \mathrm{d} \eta(\theta)=\mathbb{M}(T) .
$$

Proof of Proposition 6.8. The claim 22 follows immediately since by Theorem 6.2 one has

$$
\mu_{T}(e) \leqslant \int_{\Theta} \mathbb{M}(\llbracket \theta \rrbracket\llcorner e) \mathrm{d} \eta(\theta)
$$

for every Borel set $e \subset \mathbb{R}^{n}$, and according to 21] the latter estimate becomes an equality for $e:=\mathbb{R}^{n}$. 
Since $\mu_{T}=\mu_{\llbracket \theta \|} \otimes \eta$, the convergence $f_{v} \rightarrow g$ in $L^{1}\left(\mu_{T}\right)$ as $v \rightarrow \infty$ implies $f_{v} \rightarrow g$ in $L^{1}\left(\mu_{\|\theta\|}\right)$ for $\eta$-a.e. $\theta \in \Theta$. We use this observation to prove the last claim (23). For this purpose let $\left\{f_{v}\right\}$ be a sequence of smooth functions which converge to $1_{e}$ in $L^{1}\left(\mu_{T}\right)$ as $v \rightarrow \infty$. Since $\mu_{T}$ has finite total mass, $T\left\llcorner e(\omega):=\lim _{v} T\left(f_{v} \wedge \omega\right)\right.$. But

$$
T\left(f_{v} \wedge \omega\right)=\int_{\Theta} \llbracket \theta \rrbracket\left(f_{v} \wedge \omega\right) \mathrm{d} \eta(\theta)=\int_{\Theta}\left(\int_{\theta} f_{v}(\xi)\left\langle\omega(\xi), \tau_{\theta}(\xi)\right\rangle \mathrm{d} \mu_{\llbracket \theta \rrbracket}(\xi)\right) \mathrm{d} \eta(\theta) .
$$

As we just observed, for $\eta$-a.e. $\theta$ one has

$$
\int_{\theta} f_{\nu}(\xi)\left\langle\omega(\xi), \tau_{\theta}(\xi)\right\rangle \mathrm{d} \mu_{\llbracket \theta \rrbracket}(\xi) \rightarrow \int_{\theta \cap e}\left\langle\omega(\xi), \tau_{\theta}(\xi)\right\rangle \mathrm{d} \mu_{\llbracket \theta \rrbracket}(\xi)=: \llbracket \theta \rrbracket\llcorner e(\omega)
$$

as $v \rightarrow \infty$. Moreover,

$$
\left|\int_{\theta} f_{\nu}(\xi)\left\langle\omega(\xi), \tau_{\theta}(\xi)\right\rangle \mathrm{d} \mu_{\llbracket \theta \rrbracket}(\xi)\right| \leqslant\|\omega\|_{\infty}\left|\int_{\theta} f_{\nu} \mathrm{d} \mu_{\llbracket \theta \rrbracket}\right|
$$

and for $\eta$-a.e. $\theta$ one has $\int_{\theta} f_{v} \mathrm{~d} \mu_{\llbracket \theta \rrbracket} \rightarrow \mu_{\llbracket \theta \rrbracket}(e)$ as $v \rightarrow \infty$. Hence,

$$
\left|\int_{\theta} f_{v}(\xi)\left\langle\omega(\xi), \tau_{\theta}(\xi)\right\rangle \mathrm{d} \mu_{\llbracket \theta \rrbracket}\right| \leqslant 2\|\omega\|_{\infty} \mu_{\llbracket \theta \rrbracket}(e) .
$$

Since

$$
\int_{\Theta} \mu_{\llbracket \theta \rrbracket}(e) \mathrm{d} \eta(\theta) \leqslant \int_{\Theta} \mathbb{M}(\llbracket \theta \rrbracket) \mathrm{d} \eta(\theta) \leqslant \mathbb{M}(T),
$$

the functions $\theta \mapsto\left|\int_{\theta} f_{\mathcal{V}}\left\langle\omega, \tau_{\theta}\right\rangle \mathrm{d} \mu_{\llbracket \theta \rrbracket}\right|$ are also bounded by a function in $L^{1}(\eta)$. Hence by the Lebesgue convergence theorem, we obtain the desired result 23].

\section{Mass estimates}

We first state the following technical lemma which is practically contained in the proof of the rectifiability theorem for currents.

Lemma 7.1 Let $T$ be a $k$-dimensional real flat chain with finite mass, and set

$$
\theta_{T}(x):=\Theta_{k}^{*}\left(\mu_{T}, x\right), \quad \Sigma_{T}:=\left\{x \in \mathbb{R}^{n}: 0<\theta_{T}(x)<+\infty\right\} .
$$

Then $\Sigma_{T}$ is countably $\left(\mathcal{H}^{k}, k\right)$-rectifiable, and for $\mathcal{H}^{k}$-a.e. $x \in \Sigma_{T}$ one has

$$
\Theta_{k}^{*}\left(\mu_{T}, x\right)=\Theta_{k *}\left(\mu_{T}, x\right),
$$

while

$$
\mu_{T}\left\llcorner\Sigma=\theta_{T} \mathcal{H}^{k}\left\llcorner\left(\Sigma_{T} \cap \Sigma\right)\right.\right.
$$

for every countably $\left(\mathcal{H}^{k}, k\right)$-rectifiable set $\Sigma \subset \mathbb{R}^{n}$. 
Proof. We first claim

$$
\mathcal{H}^{k}\left\llcorner\Sigma_{T} \ll \varphi:=\mu_{T}\left\llcorner\Sigma_{T}\right.\right.
$$

In fact, if $e \subset \Sigma_{T}$, then

$$
e=\bigcup_{j=1}^{\infty} e^{j}, \quad \text { where } e^{j}:=\left\{x \in e: \Theta_{k}^{*}\left(\mu_{T}, x\right) \geqslant 1 / j\right\},
$$

and thus $\mu_{T}(e)=0$ implies by [2, Theorem 2.56] the estimate

$$
\mathcal{H}^{k}\left(e^{j}\right) \leqslant j \mu_{T}\left(e^{j}\right) \leqslant j \mu_{T}(e)=0,
$$

hence $\mathcal{H}^{k}(e)=0$, proving the claim.

Assume now that $e \subset \Sigma_{T}$ is purely $\left(\mathcal{H}^{k}, k\right)$-unrectifiable. Then $\mu_{T}(e)=0$ by [21, Theorem 3.1], and hence $\mathcal{H}^{k}(e)=0$, which proves the countable $\left(\mathcal{H}^{k}, k\right)$-rectifiability of $\Sigma_{T}$.

Observe now that $\varphi=1_{\Sigma_{T}} \mu_{T}$, and hence

$$
\frac{\varphi\left(B_{r}(x)\right)}{\mu_{T}\left(B_{r}(x)\right)} \rightarrow 1
$$

as $r \rightarrow 0^{+}$for $\mu_{T}$-a.e. $x \in \Sigma_{T}$, and hence also for $\mathcal{H}^{k}$-a.e. $x \in \Sigma_{T}$. Since

$$
\frac{\varphi\left(B_{r}(x)\right)}{\omega_{k} r^{k}}=\frac{\varphi\left(B_{r}(x)\right)}{\mu_{T}\left(B_{r}(x)\right)} \cdot \frac{\mu_{T}\left(B_{r}(x)\right)}{\omega_{k} r^{k}},
$$

we get

$$
\Theta_{k}^{*}(\varphi, x)=\Theta_{k}^{*}\left(\mu_{T}, x\right) \text { and } \Theta_{k *}(\varphi, x)=\Theta_{k *}\left(\mu_{T}, x\right)
$$

for $\mathcal{H}^{k}$-a.e. $x \in \Sigma_{T}$.

We now claim

$$
\varphi \ll \mathcal{H}^{k}\left\llcorner\Sigma_{T}\right.
$$

In fact, if $e \subset \Sigma_{T}$, then

$$
e=\bigcup_{j=1}^{\infty} e_{j}, \quad \text { where } e_{j}:=\left\{x \in e: \Theta_{k}^{*}\left(\mu_{T}, x\right) \leqslant j\right\} .
$$

Hence, $\mathcal{H}^{k}(e)=0$ implies by [2, Theorem 2.56] the estimate

$$
\mu_{T}\left(e_{j}\right) \leqslant 2^{k} j \mathcal{H}^{k}\left(e_{j}\right) \leqslant 2^{k} j \mathcal{H}^{k}(e)=0,
$$

and therefore $\mu_{T}(e)=0$, proving $\varphi \ll \mathcal{H}^{k}\left\llcorner\Sigma_{T}\right.$.

Since 25] implies $\Theta_{k}^{*}(\varphi, x)=\Theta_{k *}(\varphi, x)$ for $\mathcal{H}^{k}$-a.e. $x \in \Sigma_{T}$, we deduce from 24] that

$$
\Theta_{k}^{*}\left(\mu_{T}, x\right)=\Theta_{k *}\left(\mu_{T}, x\right) \quad \text { for } \mathcal{H}^{k} \text {-a.e. } x \in \Sigma_{T} .
$$

Finally, to show the last claim of the statement being proven, it is enough to prove it for an arbitrary countably $\left(\mathcal{H}^{k}, k\right)$-rectifiable set $\Sigma \subset \mathbb{R}^{n}$ satisfying $\mathcal{H}^{k}(\Sigma)<+\infty$. Clearly,

$$
\mu_{T}\left(\Sigma \cap \Sigma_{T}\right)=\varphi(\Sigma)=\int_{\Sigma_{T}} \theta_{T} \mathrm{~d} \mathcal{H}^{k}
$$


We now write

$$
\mu_{T}\left(\Sigma \backslash \Sigma_{T}\right)=\mu_{T}\left(\Sigma \cap\left\{\Theta_{k}^{*}\left(\mu_{T}, x\right)=+\infty\right\}\right)+\mu_{T}\left(\Sigma \cap\left\{\Theta_{k}^{*}\left(\mu_{T}, x\right)=0\right\}\right) .
$$

But

$$
\begin{aligned}
\mathcal{H}^{k}\left(\left\{\Theta_{k}^{*}\left(\mu_{T}, x\right)=+\infty\right\}\right) & =\mathcal{H}^{k}\left(\bigcap_{j=1}^{\infty}\left\{\Theta_{k}^{*}\left(\mu_{T}, x\right) \geqslant j\right\}\right)=\inf _{j} \mathcal{H}^{k}\left(\left\{\Theta_{k}^{*}\left(\mu_{T}, x\right) \geqslant j\right\}\right) \\
& \leqslant \inf _{j} \frac{1}{j} \mu_{T}\left(\left\{\Theta_{k}^{*}\left(\mu_{T}, x\right) \geqslant j\right\}\right) \leqslant \inf _{j} \mu_{T}\left(\mathbb{R}^{n}\right) / j=0,
\end{aligned}
$$

hence $\left.\left.\mu_{T}\left\{\Theta_{k}^{*}\left(\mu_{T}, x\right)=+\infty\right\}\right)\right)=0$ by [25]. On the other hand,

$$
\begin{aligned}
\mu_{T}\left(\Sigma \cap\left\{\Theta_{k}^{*}\left(\mu_{T}, x\right)=0\right\}\right) & =\mu_{T}\left(\bigcap_{j=1}^{\infty}\left\{x \in \Sigma: \Theta_{k}^{*}\left(\mu_{T}, x\right) \leqslant 1 / j\right\}\right) \\
& =\inf _{j} \mu_{T}\left(\left\{x \in \Sigma: \Theta_{k}^{*}\left(\mu_{T}, x\right) \leqslant 1 / j\right\}\right) \leqslant \inf _{j} \frac{2^{k}}{j} \mathcal{H}^{k}(\Sigma)=0 .
\end{aligned}
$$

Putting the above estimates together, we get $\mu_{T}\left(\Sigma \cap \Sigma_{T}\right)=0$, which together with 26) concludes the proof of the last claim.

Given a transport $\eta$, we define the transiting mass function $a_{\eta}: \mathbb{R}^{n} \rightarrow \mathbb{R}$ by setting

$$
a_{\eta}(x):=\eta(\{\theta \in \Theta: x \in \theta\}) .
$$

In other words, $a_{\eta}(x)$ measures the number of people passing through the point $x \in \mathbb{R}^{n}$. We may now state the following result.

LEMMA 7.2 Let $T$ be a one-dimensional real flat chain with compact support and $\mathbb{M}(T)<+\infty$. Let $\eta$ be given by Theorem 6.3 and let $\theta_{T}$ be defined as in Lemma 7.1. Then

(i) $a_{\eta}$ is u.s.c.;

(ii) if $T$ is acyclic, then $\theta_{T}(x)=a_{\eta}(x)$ for $\mathcal{H}^{1}$-a.e. $x \in \mathbb{R}^{n}$.

Proof. For each $x \in \mathbb{R}^{n}$ define the function $1_{x}: \Theta \rightarrow \mathbb{R}$ by the formula

$$
1_{x}(\sigma):= \begin{cases}1 & \text { if } x \in \sigma, \\ 0 & \text { otherwise }\end{cases}
$$

Clearly, $1_{x}$ is u.s.c. To prove (i), it is therefore enough to observe that

$$
a_{\eta}(x)=\int_{\Theta} 1_{x}(\sigma) \mathrm{d} \eta(\sigma)
$$

and to apply Fatou's lemma.

To prove (ii), it is enough to show that for an acyclic $T$ one has

(A) for each countably $\left(\mathcal{H}^{1}, 1\right)$-rectifiable set $\Sigma \subset \mathbb{R}^{n}$ one has $\theta_{T}(x)=a_{\eta}(x)$ for $\mathcal{H}^{1}$-a.e. $x \in \Sigma$;

(B) $\theta_{T}(x) \geqslant a_{\eta}(x) / 2$ for $\mathcal{H}^{1}$-a.e. $x \in \mathbb{R}^{n}$. 
In fact, the set $\Sigma_{T}$ is countably $\left(\mathcal{H}^{1}, 1\right)$-rectifiable by Lemma 7.1, and hence $\theta_{T}(x)=a_{\eta}(x)$ for $\mathcal{H}^{1}$-a.e. $x \in \Sigma_{T}$ by (A). On the other hand, for $\mathcal{H}^{1}$-a.e. $x \notin \overline{\Sigma_{T}}$ one has $\theta_{T}(x)=0$, and hence $a_{\eta}(x)=0$ by (B), which shows (ii).

We now prove (A) and (B). To show (A), recall that due to Theorem 6.3, as $\eta$-a.e. $\sigma^{\prime} \in \Theta$ is an arc, for all $\sigma \in \Theta$ one has

$$
\begin{aligned}
\mu_{T}(\sigma) & =\int_{\Theta} \mathbb{M}\left(\llbracket \sigma^{\prime} \rrbracket\llcorner\sigma) \mathrm{d} \eta\left(\sigma^{\prime}\right)=\int_{\Theta} \mathcal{H}^{1}\left(\sigma^{\prime} \cap \sigma\right) \mathrm{d} \eta\left(\sigma^{\prime}\right)=\int_{\Theta}\left(\int_{\sigma} 1_{x}\left(\sigma^{\prime}\right) \mathrm{d} \mathcal{H}^{1}(x)\right) \mathrm{d} \eta\left(\sigma^{\prime}\right)\right. \\
& =\int_{\sigma}\left(\int_{\Theta} 1_{x}\left(\sigma^{\prime}\right) \mathrm{d} \eta\left(\sigma^{\prime}\right)\right) \mathrm{d} \mathcal{H}^{1}(x)=\int_{\sigma} a_{\eta} \mathrm{d} \mathcal{H}^{1} .
\end{aligned}
$$

On the other hand, by Lemma 7.1 .

$$
\mu_{T}(\sigma)=\int_{\sigma} \theta_{T} \mathrm{~d} \mathcal{H}^{1}
$$

which proves

$$
\int_{\sigma} \theta_{T} \mathrm{~d} \mathcal{H}^{1}=\int_{\sigma} a_{\eta} \mathrm{d} \mathcal{H}^{1}
$$

for every Lipschitz curve $\sigma$. This clearly implies (A).

To prove (B) fix $x \in \mathbb{R}^{n}$ and let $\varepsilon>0$. Consider the sets

$$
\begin{aligned}
A(x) & :=\{\sigma \in \Theta: x \in \sigma\}, \\
A_{\rho}(x) & :=\left\{\sigma \in A(x): \mathbb{M}\left(\llbracket \sigma \rrbracket\left\llcorner B_{\rho}(x)\right) \geqslant \rho\right\},\right. \\
A_{\rho}^{\prime}(x) & :=A(x) \backslash A_{\rho}(x), \\
C_{\rho}^{+}(x) & :=\left\{\sigma \in \Theta: \sigma(0) \in B_{\rho}(x)\right\}, \\
C_{\rho}^{-}(x) & :=\left\{\sigma \in \Theta: \sigma(1) \in B_{\rho}(x)\right\}, \\
C_{\rho}(x) & :=C_{\rho}^{+}(x) \cap C_{\rho}^{-}(x) .
\end{aligned}
$$

One has $\eta\left(C_{\rho}^{ \pm}(x)\right)=\left(t_{0,1}\right)_{\#} \eta\left(B_{\rho}(x)\right)=(\partial T)^{ \pm}\left(B_{\rho}(x)\right)$ and hence

$$
\eta\left(C_{\rho}(x)\right) \leqslant(\partial T)^{+}\left(B_{\rho}(x)\right) \wedge(\partial T)^{-}\left(B_{\rho}(x)\right) \rightarrow 0 \quad \text { as } \rho \rightarrow 0^{+} .
$$

In particular there exists $\delta>0$ such that $\eta\left(C_{\rho}(x)\right)<\varepsilon$ for every $\rho<\delta$.

In view of Theorem 6.3, $\eta$-a.e. $\sigma \in \Theta$ is an arc. Hence for $\eta$-a.e. $\sigma \in A(x)$ if either $\sigma(0) \notin$ $B_{\rho}(x)$ or $\sigma(1) \notin B_{\rho}(x)$ then $\mathbb{M}\left(\llbracket \sigma \rrbracket\left\llcorner B_{\rho}(x)\right) \geqslant \rho\right.$. This proves that $\eta\left(A_{\rho}^{\prime}(x) \backslash C_{\rho}(x)\right)=0$. Hence $\eta\left(A_{\rho}^{\prime}(x)\right) \leqslant \varepsilon$, and consequently $\eta\left(A_{\rho}(x)\right)=\eta(A(x))-\eta\left(A_{\rho}^{\prime}(x)\right) \geqslant a_{\eta}(x)-\varepsilon$.

To conclude, note that

$$
\begin{aligned}
\frac{\mathbb{M}\left(T\left\llcorner B_{\rho}(x)\right)\right.}{2 \rho} & =\frac{1}{2 \rho} \int_{\Theta} \mathbb{M}\left(\llbracket \sigma \rrbracket\left\llcorner B_{\rho}(x)\right) \mathrm{d} \eta(\sigma) \geqslant \frac{1}{2 \rho} \int_{A_{\rho}(x)} \mathbb{M}\left(\llbracket \sigma \rrbracket\left\llcorner B_{\rho}(x)\right) \mathrm{d} \eta(\sigma)\right.\right. \\
& \geqslant \frac{\rho\left(a_{\eta}(x)-\varepsilon\right)}{2 \rho}=\left(a_{\eta}(x)-\varepsilon\right) / 2,
\end{aligned}
$$

so that for $\mathcal{H}^{1}$-a.e. $x \in \mathbb{R}^{n}$ one has $\theta_{T}(x) \geqslant\left(a_{\eta}(x)-\varepsilon\right) / 2$, and since this is true for every $\varepsilon>0$, the conclusion (B) follows. 
THEOREM 7.3 If $T$ is an acyclic one-dimensional normal current, then

$$
\theta_{T}(x) \leqslant \frac{1}{2} \mathbb{M}(\partial T) \quad \text { for } \mathcal{H}^{1} \text {-a.e. } x \in \mathbb{R}^{n} .
$$

Proof. If $T$ is an acyclic normal current, then from Theorem 6.3 one has $T=T_{\eta}$ for some transport $\eta$ such that $\eta(0)=(\partial T)^{+}$. Then, as $\Sigma_{T}$ is countably $\left(\mathcal{H}^{1}, 1\right)$-rectifiable by Lemma 7.1 it follows from Lemma 7.2 ii) that $\theta_{T}(x)=a_{\eta}(x)$ for $\mathcal{H}^{1}$-a.e. $x \in \Sigma_{T}$. But

$$
a_{\eta}(x) \leqslant \eta(\Theta) \leqslant \eta(0)\left(\mathbb{R}^{n}\right)=\frac{1}{2} \mathbb{M}(\partial T),
$$

and thus $\theta_{T}(x) \leqslant \mathbb{M}(\partial T) / 2$ for $\mathcal{H}^{1}$-a.e. $x \in \Sigma_{T}$. On the other hand, $\mathcal{H}^{1}$-a.e. on $\mathbb{R}^{n} \backslash \Sigma_{T}$ one has $\theta_{T}=0$ (since it has been shown in the proof of Lemma 7.1 that $\mathcal{H}^{1}\left(\left\{\theta_{T}=+\infty\right\}\right)=0$ ), which concludes the proof.

The assertion below may be regarded as a version of the Sobolev-Poincaré inequality for onedimensional real flat chains.

THEOREM 7.4 Let $T$ be an acyclic one-dimensional real flat chain of finite mass and assume that $S \leqslant T$. Then

$$
\mathbb{M}^{\beta}(S) \leqslant \frac{1}{2^{\beta-\alpha}} \mathbb{M}^{\alpha}(S) \mathbb{M}(\partial T)^{\beta-\alpha} \quad \text { for all } \alpha \in[0,1], \beta \in[\alpha, 1] .
$$

In particular,

$$
\mathbb{M}(S) \leqslant \frac{1}{2^{1-\alpha}} \mathbb{M}^{\alpha}(S) \mathbb{M}(\partial T)^{1-\alpha} \quad \text { for every } \alpha \in[0,1] .
$$

Proof. By Theorem 7.3 the claim is easily proven when $S$ is rectifiable and $T$ is normal. In fact, in this case one may consider $\Sigma_{S} \subset \Sigma_{T}, \theta_{S} \leqslant \theta_{T}$. Therefore

$$
\begin{aligned}
\mathbb{M}^{\beta}(S) & =\int_{\Sigma_{S}} \theta_{S}^{\beta} \mathrm{d} \mathcal{H}^{1}=(\mathbb{M}(\partial T) / 2)^{\beta} \int_{\Sigma_{S}}\left(\frac{\theta_{S}}{\mathbb{M}(\partial T) / 2}\right)^{\beta} \mathrm{d} \mathcal{H}^{1} \\
& \leqslant(\mathbb{M}(\partial T) / 2)^{\beta} \int_{\Sigma_{S}}\left(\frac{\theta_{S}}{\mathbb{M}(\partial T) / 2}\right)^{\alpha} \mathrm{d} \mathcal{H}^{1}=\frac{1}{2^{\beta-\alpha}} \mathbb{M}^{\alpha}(S) \mathbb{M}(\partial T)^{\beta-\alpha} .
\end{aligned}
$$

To prove the claim in the general case, it is enough to note that we may assume $\alpha<1$ (otherwise there is nothing to prove), and then the hypothesis $\mathbb{M}(T)<+\infty$ provides $\mathbb{M}(S)<+\infty$, and hence, by Theorem 2.1. $S$ is rectifiable. One may also suppose $\mathbb{M}(\partial T)<+\infty$ (otherwise there is still nothing to prove), which guarantees that $T$ is normal. Hence the assertion follows.

\section{Existence of solutions}

To illustrate the developed technique we prove the existence of solutions to Problem 11 in an important particular case when $H(\cdot)$ is concave.

THEOREM 8.1 Let $\varphi^{ \pm}$be finite nonnegative Borel measures with compact support in $\mathbb{R}^{n}$, satisfying $\varphi^{+}\left(\mathbb{R}^{n}\right)=\varphi^{-}\left(\mathbb{R}^{n}\right)$. Assume also the function $H$ to be concave, and $H(l) \rightarrow+\infty$ as $l \rightarrow+\infty, A>0, \alpha<1$, and either $\alpha>\beta \vee \delta$, or $\alpha=\beta>\delta$, but $A>B$. Then the functional $\mathfrak{F}$ attains its minimum value on the set of pairs $(T, S)$ of one-dimensional real flat chains of finite mass which satisfy (3). In other words, in this case Problem[1]admits solutions. 
Proof. Assume the existence of a pair $\left(T_{0}, S_{0}\right)$ of one-dimensional real flat chains of finite mass such that $\partial\left(T_{0}+S_{0}\right)=\varphi^{+}-\varphi^{-}$and

$$
\mathfrak{F}\left(T_{0}, S_{0}\right)<+\infty
$$

(otherwise $\mathfrak{F} \equiv+\infty$, and hence there is nothing to prove). Here and below for the sake of brevity we denote $\phi:=\varphi^{+}-\varphi^{-}$.

We may also assume that $H \not \equiv+\infty$ on $(0,+\infty)$. In fact, in the opposite case Problem 1 admits a trivial solution $(T, 0)$, where $T$ is a real flat chain minimizing $\mathbb{M}^{\alpha}$ among all one-dimensional real flat chains of finite mass satisfying $\partial T=\phi$.

We divide the proof into several steps.

Step 1. We first show the existence of a minimizing sequence $\left\{\left(T_{v}, S_{v}\right)\right\}$ for the functional $\mathfrak{F}$ of pairs of real rectifiable currents which satisfy the condition 11 , have uniformly bounded masses and also satisfy condition $\mu_{T_{v}} \wedge \mu_{S_{v}}=0$.

Let $\left\{\left(T_{v}^{\prime}, S_{v}^{\prime}\right)\right\}$ be an arbitrary minimizing sequence for $\mathfrak{F}$ which satisfies $(1)$. Then $\mathfrak{F}\left(T_{v}^{\prime}, S_{v}^{\prime}\right)<$ $+\infty$. In view of the assumption on $H$ we have $\mathbb{M}^{\delta}\left(S_{v}^{\prime}\right)<+\infty$, and hence one may apply Theorem 10.2 below, which gives rectifiability of $S_{v}^{\prime}$. Since $\mathbb{M}^{\alpha}\left(T_{v}^{\prime}\right)<+\infty$, according to the same theorem also $T_{v}^{\prime}$ is rectifiable. In view of Lemma 4.6 we may assume without loss of generality that $\mu_{T_{v}^{\prime}} \wedge \mu_{S_{v}^{\prime}}=0$. In other words, for every $v \in \mathbb{N}$ there is a Borel set $E_{v} \subset \mathbb{R}^{n}$ such that

$$
T_{v}^{\prime}=T_{v}^{\prime}\left\llcorner E_{v} \quad S_{v}^{\prime}=S_{v}^{\prime}\left\llcorner\left(\mathbb{R}^{n} \backslash E_{v}\right) .\right.\right.
$$

According to Proposition 3.12 there is a cycle $C_{v} \leqslant T_{v}^{\prime}+S_{v}^{\prime}$ such that the current $T_{v}^{\prime}+S_{v}^{\prime}-C_{v}$ is acyclic. Setting

$$
T_{\nu}:=T_{\nu}^{\prime}-C_{\nu}\left\llcorner E_{\nu}, \quad S_{\nu}:=S_{v}^{\prime}-C_{\nu}\left\llcorner\left(\mathbb{R}^{n} \backslash E_{v}\right),\right.\right.
$$

we get $T_{v}+S_{v}=T_{v}^{\prime}+S_{v}^{\prime}-C_{v}$, and hence

$$
\partial\left(T_{\nu}+S_{v}\right)=\partial\left(T_{v}^{\prime}+S_{v}^{\prime}\right)=\phi .
$$

On the other hand, from $C_{v} \leqslant T_{v}^{\prime}+S_{v}^{\prime}$ one gets

$$
C_{v}\left\llcorner E_{v} \leqslant\left(T_{v}^{\prime}+S_{v}^{\prime}\right)\left\llcorner E_{v}=T_{v}^{\prime} .\right.\right.
$$

Due to Remark 3.6. one has $T_{\nu} \leqslant T_{\nu}^{\prime}$. Analogously, $S_{\nu} \leqslant S_{\nu}^{\prime}$, and hence, applying Lemma 3.7 , we get $\mathfrak{F}\left(T_{v}, S_{v}\right) \leqslant \mathfrak{F}\left(T_{v}^{\prime}, S_{v}^{\prime}\right)$, i.e. $\left\{\left(T_{v}, S_{v}\right)\right\}$ is still a minimizing sequence for Problem 1 Let $R_{v}:=T_{v}+S_{\nu}$. In view of acyclicity of $R_{v}$ we may apply Theorem 7.4 to get

$$
\mathbb{M}\left(T_{\nu}\right) \leqslant \frac{1}{2^{1-\alpha}} \mathbb{M}^{\alpha}\left(T_{\nu}\right) \mathbb{M}\left(\partial R_{\nu}\right)^{1-\alpha} \leqslant \frac{1}{2^{1-\alpha}} \mathbb{M}^{\alpha}\left(T_{\nu}^{\prime}\right)|\phi|\left(\mathbb{R}^{n}\right)^{1-\alpha},
$$

taking into account that $\mathbb{M}^{\alpha}\left(T_{v}\right) \leqslant \mathbb{M}^{\alpha}\left(T_{v}^{\prime}\right)$ by Lemma 3.7, since $T_{v} \leqslant T_{v}^{\prime}$, and that $\mathbb{M}\left(\partial R_{v}\right)=$ $|\phi|\left(\mathbb{R}^{n}\right)$. In the same way we get the estimate

$$
\mathbb{M}\left(S_{v}\right) \leqslant \frac{1}{2^{1-\delta}} \mathbb{M}^{\delta}\left(S_{v}^{\prime}\right)|\phi|\left(\mathbb{R}^{n}\right)^{1-\delta} .
$$

On the other hand, since $\mathfrak{F}\left(T_{\nu}, S_{v}\right) \leqslant \mathfrak{F}\left(T_{0}, S_{0}\right)$, for some $C^{\prime}>0$ and for all $v \in \mathbb{N}$ we have the estimates

$$
\mathbb{M}^{\alpha}\left(T_{v}^{\prime}\right) \leqslant C^{\prime}, \quad \mathbb{M}^{\delta}\left(S_{v}^{\prime}\right) \leqslant C^{\prime},
$$


because $A>0$ and the functon $H$ is unbounded. Combining the above estimates with (27) and (28), we conclude that both $T_{v}$ and $S_{v}$, and hence also $R_{v}$, have uniformly bounded masses $\mathbb{M}$.

Step 2. For every $d \geqslant 0$ and every real flat chain $R$ we let

$$
S_{d}(R):=R\left\llcorner\left(\Sigma_{R} \cap\left\{\theta_{R} \geqslant d\right\}\right), \quad T_{d}(R):=R-S_{d}(R) .\right.
$$

If $C \in\left[H_{+}^{\prime}\left(\mathbb{M}^{\delta}(S)\right), H_{-}^{\prime}\left(\mathbb{M}^{\delta}(S)\right)\right]$, where $H_{ \pm}^{\prime}$ are the left and right derivatives of $H$ respectively, and $l \geqslant 0$, we also define

$$
F[l, C](R):=\mathbb{M}^{\alpha}\left(T_{d}(R)\right)+B \mathbb{M}^{\beta}\left(S_{d}(R)\right)+H(l)+C\left(\mathbb{M}^{\delta}\left(S_{d}(R)\right)-l\right) .
$$

Let $d \geqslant 0$ be such that $A t^{\alpha}>B t^{\beta}+C t^{\delta}$ when $t \in(d,+\infty)$, and $A t^{\alpha}<B t^{\beta}+C t^{\delta}$ when $t \in(0, d)$ (here and below we assume $(0, d):=\emptyset$ if $d=0)$. Then clearly

$$
\mathfrak{F}\left(T_{d}(R), S_{d}(R)\right)=F\left[\mathbb{M}^{\delta}\left(S_{d}(R)\right), C\right](R),
$$

while

$$
\mathfrak{F}\left(T_{d}(R), S_{d}(R)\right) \leqslant F[l, C](R) \quad \text { for all } l \geqslant 0 .
$$

Consider a minimizing sequence for the functional $\mathfrak{F}$ constructed in Step 1 . Note that since $\phi$ is concentrated on some ball in $\mathbb{R}^{n}$, one may assume that all $T_{v}$ and $S_{v}$ (and hence also $R_{v}$ ) are concentrated on the same ball (otherwise, projecting the latter currents to this ball will not change the boundary of their sum while not increasing any of the masses $\mathbb{M}^{\lambda}, \lambda \in[0,1]$, and hence, not increasing the value of $\mathfrak{F})$. Since the masses $\mathbb{M}\left(R_{v}\right)$ are uniformly bounded, and $\partial R_{v}=\phi$, up to a subsequence we have $R_{v} \stackrel{\mathcal{F}}{\rightarrow} R$ as $v \rightarrow \infty$ for some real one-dimensional flat chain $R$ satisfying $\partial R=\phi$.

Set $l_{v}:=\mathbb{M}^{\delta}\left(S_{v}\right)$, and choose an arbitrary $C_{v} \in\left[H_{+}^{\prime}\left(l_{v}\right), H_{-}^{\prime}\left(l_{v}\right)\right]$. Observe that $C_{v} \neq 0$ due to the assumption on $H$. Without loss of generality we may assume that up to a subsequence, $l_{v} \rightarrow l$ and $C_{v} \rightarrow C$ for some $l \in[0,+\infty]$ and $C \in[0,+\infty]$ as $v \rightarrow \infty$. We consider separately two possible situations.

CASE $l>0$. Then $C<+\infty$. Note that the numbers $l_{v}$ are uniformly bounded, since otherwise up to a subsequence, $H\left(l_{v}\right) \rightarrow+\infty$, and hence $\mathfrak{F}\left(T_{v}, S_{v}\right) \rightarrow+\infty$ as $v \rightarrow \infty$, contrary to the estimate $\mathfrak{F}\left(T_{v}, S_{v}\right) \leqslant \mathfrak{F}\left(T_{0}, S_{0}\right)<+\infty$ for all sufficiently large $v$ (because the sequence $\left\{\left(T_{v}, S_{v}\right)\right\}$ is minimizing). Therefore, $l<+\infty$, which also implies that $C>0$ (otherwise $H_{+}^{\prime}(l)=0$, which would mean, in view of concavity of $H$, that $H(t)=H(l)$ for all $t \geqslant l$, contrary to the assumption on unboundedness of $H$ ). We finally remark that $C \in\left[H_{+}^{\prime}(l), H_{-}^{\prime}(l)\right]$, since the functions $H_{ \pm}^{\prime}$ are lower and upper semicontinuous respectively.

In view of Lemma 10.5 we may assume without loss of generality that

$$
\begin{array}{ll}
\theta_{S_{v}}(x)=\theta_{R_{v}}(x) \geqslant d_{v} & \text { for } \mathcal{H}^{1} \text {-a.e. } x \in \Sigma_{S_{v}}, \\
\theta_{T_{v}}(x)=\theta_{R_{v}}(x)<d_{v} & \text { for } \mathcal{H}^{1} \text {-a.e. } x \in \Sigma_{T_{v}},
\end{array}
$$

where the numbers $d_{v}>0$ depend only on $\alpha, \beta, \delta, A, B$ and $C_{v}$ and satisfy the relationships

$$
\begin{array}{ll}
A t^{\alpha}<B t^{\beta}+C_{v} t^{\delta}, & t \in\left(0, d_{v}\right), \\
A t^{\alpha}>B t^{\beta}+C_{\nu} t^{\delta}, & t \in\left(d_{v},+\infty\right) .
\end{array}
$$


In other words, in view of rectifiability of $S_{v}$ one has $S_{v}=S_{d_{v}}\left(R_{v}\right)$, and hence $T_{v}=T_{d_{v}}\left(R_{v}\right)$. Thus, by 29,

$$
\liminf _{v} \mathfrak{F}\left(T_{v}, S_{v}\right)=\liminf _{v} \mathfrak{F}\left(T_{d_{v}}\left(R_{v}\right), S_{d_{v}}\left(R_{v}\right)\right)=\liminf _{v} F\left[l_{v}, C_{v}\right]\left(R_{v}\right)
$$

In view of rectifiability of $T_{v}, S_{v}$ and $R_{v}$, and because $\mu_{T_{v}} \wedge \mu_{S_{v}}=0$, one can write

$$
\begin{aligned}
& F\left[l_{\nu}, C_{\nu}\right]\left(R_{\nu}\right)=\int_{\Sigma_{R_{v}} \cap\left\{\theta_{R_{v}}(x)<d_{\nu}\right\}} A \theta_{R_{\nu}}^{\alpha}(x) \mathrm{d} \mathcal{H}^{1}(x) \\
& +\int_{\Sigma_{R_{v}} \cap\left\{\theta_{R_{v}}(x) \geqslant d_{v}\right\}}\left(B \theta_{R_{v}}^{\beta}(x)+C_{v} \theta_{R_{v}}^{\delta}(x)\right) \mathrm{d} \mathcal{H}^{1}(x)+H\left(l_{v}\right)-C_{v} l_{v} \\
& =\int_{\Sigma_{R_{v}}} g_{\nu}\left(\theta_{R_{v}}(x)\right) \mathrm{d} \mathcal{H}^{1}(x)+H\left(l_{v}\right)-C_{\nu} l_{\nu},
\end{aligned}
$$

where

$$
g_{v}(t):=\left(A t^{\alpha}\right) \wedge\left(B t^{\beta}+C_{v} t^{\delta}\right),
$$

since $f_{v}(t):=A t^{\alpha}-B t^{\beta}-C_{v} t^{\delta}>0$ when $t>d_{v}$, and $f_{v}(t)<0$ when $t \in\left(0, d_{v}\right)$.

For all $\varepsilon>0$ and sufficiently large $v \in \mathbb{N}$ one has

$$
g_{v}(t) \geqslant g^{\varepsilon}(t):=\left(A t^{\alpha}\right) \wedge\left(B t^{\beta}+(C-\varepsilon) t^{\delta}\right) \text { for all } t \geqslant 0 .
$$

Therefore,

$$
\liminf _{v} F\left[l_{v}, C_{v}\right]\left(R_{v}\right) \geqslant \liminf \int_{\Sigma_{R_{v}}} g^{\varepsilon}\left(\theta_{R_{v}}(x)\right) \mathrm{d} \mathcal{H}^{1}(x)+H(l)-C l .
$$

Since for $\varepsilon \in[0, C)$ the function $g^{\varepsilon}: \mathbb{R}^{+} \rightarrow \mathbb{R}^{+}$is nondecreasing and concave, and $g^{\varepsilon}(0)=0$, the functional

$$
R \mapsto \int_{\Sigma_{R}} g^{\varepsilon}\left(\theta_{R}(x)\right) \mathrm{d} \mathcal{H}^{1}(x),
$$

defined on rectifiable currents, defines an l.s.c. (in the flat norm topology) functional $\mathbb{M}^{g^{\varepsilon}}$ on the set of real flat chains according to the formula

$$
\mathbb{M}^{g^{\varepsilon}}(R):=\inf \left\{\liminf _{\nu} \int_{\Sigma_{R_{\nu}}} g^{\varepsilon}\left(\theta_{R_{\nu}}(x)\right) \mathrm{d} \mathcal{H}^{1}(x)\right\}
$$

where the infimum is taken over all sequences $\left\{R_{v}\right\}$ of real polyhedral chains converging to $R$ in the flat norm. Then

$$
\mathbb{M}^{g^{\varepsilon}}(R)=\int_{\Sigma_{R}} g^{\varepsilon}\left(\theta_{R}(x)\right) \mathrm{d} \mathcal{H}^{1}(x)
$$

if $R$ is rectifiable [22]. By definition of $\mathbb{M}^{g^{\varepsilon}}$,

$$
\mathbb{M}^{g^{\varepsilon}}(R) \leqslant \liminf _{\nu} \int_{\Sigma_{R_{\nu}}} g^{\varepsilon}\left(\theta_{R_{\nu}}(x)\right) \mathrm{d} \mathcal{H}^{1}(x) .
$$


In view of 34, we get

$$
\liminf _{\nu} F\left[l_{\nu}, C_{\nu}\right]\left(R_{\nu}\right) \geqslant \mathbb{M}^{g^{\varepsilon}}(R)+H(l)-C l .
$$

From 36 and 32 one gets the inequality

$$
\liminf _{\nu} \mathfrak{F}\left(T_{\nu}, S_{\nu}\right) \geqslant \mathbb{M}^{g^{\varepsilon}}(R)+H(l)-C l,
$$

which, in particular, implies $\mathbb{M}^{g^{\varepsilon}}(R)<+\infty$. Also,

$$
\mathbb{M}(R) \leqslant \liminf _{\nu} \mathbb{M}\left(R_{\nu}\right)=\liminf _{\nu}\left(\mathbb{M}\left(T_{\nu}\right)+\mathbb{M}\left(S_{\nu}\right)\right)<+\infty,
$$

since all $T_{v}$ and $S_{v}$ have uniformly bounded masses. Therefore, from the general theorem on rectifiability of flat chains [22], recalling the definition of $g^{\varepsilon}$ and that $\alpha<1$, we get the rectifiability of $R$. Thus, $\mathbb{M}^{g^{\varepsilon}}(R)=\int_{\Sigma_{R}} g^{\varepsilon}\left(\theta_{R}(x)\right) \mathrm{d} \mathcal{H}^{1}(x)$, and hence the inequality (37) can be rewritten as

$$
\liminf _{\nu} \mathfrak{F}\left(T_{\nu}, S_{\nu}\right) \geqslant \int_{\Sigma_{R}} g^{\varepsilon}\left(\theta_{R}(x)\right) \mathrm{d} \mathcal{H}^{1}(x)+H(l)-C l .
$$

Observe that the limit $d:=\lim _{\nu} d_{\nu}$ exists and satisfies

$$
\begin{array}{ll}
A t^{\alpha}<B t^{\beta}+C t^{\delta}, & t \in(0, d), \\
A t^{\alpha}>B t^{\beta}+C t^{\delta}, & t \in(d,+\infty) .
\end{array}
$$

In fact, denoting by $s$ the limit of an arbitrary subsequence $d_{v}$ (not relabelled), and passing to the limit in 39 , we get

$$
\begin{array}{ll}
A t^{\alpha}<B t^{\beta}+C t^{\delta}, & t \in(0, s), \\
A t^{\alpha}>B t^{\beta}+C t^{\delta}, & t \in(s,+\infty),
\end{array}
$$

and hence $s=d$. Note also that $d>0$ in view of Lemma 10.6 below.

Denote by $d_{\varepsilon}$ a number such that

$$
\begin{array}{ll}
A t^{\alpha}<B t^{\beta}+(C-\varepsilon) t^{\delta}, & t \in\left(0, d_{\varepsilon}\right), \\
A t^{\alpha}>B t^{\beta}+(C-\varepsilon) t^{\delta}, & t \in\left(d_{\varepsilon},+\infty\right) .
\end{array}
$$

Clearly, $d_{\varepsilon} \leqslant d$. Moreover, $d_{\varepsilon}>0$ if $\varepsilon \in[0, C)$, due to Lemma 10.6, while, as just proven above, $d_{\varepsilon} \rightarrow d$ as $\varepsilon \rightarrow 0^{+}$. With the above notation

$$
\int_{\Sigma_{R}} g^{\varepsilon}\left(\theta_{R}(x)\right) \mathrm{d} \mathcal{H}^{1}(x)=A \mathbb{M}^{\alpha}\left(T_{d_{\varepsilon}}(R)\right)+B \mathbb{M}^{\beta}\left(S_{d_{\varepsilon}}(R)\right)+(C-\varepsilon) \mathbb{M}^{\delta}\left(S_{d_{\varepsilon}}(R)\right),
$$

and hence

$$
\begin{aligned}
\int_{\Sigma_{R}} g^{\varepsilon}\left(\theta_{R}(x)\right) \mathrm{d} \mathcal{H}^{1}(x)= & A \mathbb{M}^{\alpha}\left(T_{d}(R)\right)+B \mathbb{M}^{\beta}\left(S_{d}(R)\right)+(C-\varepsilon) \mathbb{M}^{\delta}\left(S_{d}(R)\right) \\
& -A \mathbb{M}^{\alpha}\left(R\left\llcorner\Delta_{\varepsilon}\right)+B \mathbb{M}^{\beta}\left(R\left\llcorner\Delta_{\varepsilon}\right)+(C-\varepsilon) \mathbb{M}^{\delta}\left(R\left\llcorner\Delta_{\varepsilon}\right),\right.\right.\right.
\end{aligned}
$$


where $\Delta_{\varepsilon}:=\left\{x \in \mathbb{R}^{n}: d_{\varepsilon} \leqslant \theta_{R}(x)<d\right\}$. Using [38), we get

$$
\begin{aligned}
\liminf _{\nu} \mathfrak{F}\left(T_{\nu}, S_{\nu}\right) \geqslant & F[l, C](R)-A \mathbb{M}^{\alpha}\left(R\left\llcorner\Delta_{\varepsilon}\right)+B \mathbb{M}^{\beta}\left(R\left\llcorner\Delta_{\varepsilon}\right)\right.\right. \\
& +(C-\varepsilon) \mathbb{M}^{\delta}\left(R\left\llcorner\Delta_{\varepsilon}\right)-\varepsilon \mathbb{M}^{\delta}\left(S_{d}(R)\right),\right.
\end{aligned}
$$

because

$$
F[l, C](R)=A \mathbb{M}^{\alpha}\left(T_{d}(R)\right)+B \mathbb{M}^{\beta}\left(S_{d}(R)\right)+C \mathbb{M}^{\delta}\left(S_{d}(R)\right)+H(l)-C l .
$$

Finally, by [30, we get from (42) the inequality

$$
\begin{aligned}
\liminf _{v} \mathfrak{F}\left(T_{\nu}, S_{v}\right) \geqslant & \mathfrak{F}\left(T_{d}(R), S_{d}(R)\right)-A \mathbb{M}^{\alpha}\left(R\left\llcorner\Delta_{\varepsilon}\right)+B \mathbb{M}^{\beta}\left(R\left\llcorner\Delta_{\varepsilon}\right)\right.\right. \\
& +(C-\varepsilon) \mathbb{M}^{\delta}\left(R\left\llcorner\Delta_{\varepsilon}\right)-\varepsilon \mathbb{M}^{\delta}\left(S_{d}(R)\right)\right. \\
= & \mathfrak{F}\left(T_{d}(R), S_{d}(R)\right)-\int_{\Delta_{\varepsilon}}\left(A \theta_{R}^{\alpha}(x)-B \theta_{R}^{\beta}(x)-C \theta_{R}^{\delta}(x)\right) \mathrm{d} \mathcal{H}^{1}(x) \\
& +\varepsilon \int_{\left\{\theta_{R}(x) \geqslant d_{\varepsilon}\right\}} \theta_{R}^{\delta}(x) \mathrm{d} \mathcal{H}^{1}(x) \\
= & \mathfrak{F}\left(T_{d}(R), S_{d}(R)\right)-\int_{\Delta_{\varepsilon}}\left(A \theta_{R}^{\alpha}(x)-B \theta_{R}^{\beta}(x)-C \theta_{R}^{\delta}(x)\right) \\
& +\mathrm{d} \mathcal{H}^{1}(x)+\varepsilon \mathbb{M}^{\delta}\left(S_{d_{\varepsilon}}(R)\right) .
\end{aligned}
$$

The estimates $\sup _{\varepsilon \in(0, C)} \mathbb{M}^{g^{\varepsilon}}(R)<+\infty$ and (40) imply

$$
A \mathbb{M}^{\alpha}\left(T_{d}(R)\right)=\sup _{\varepsilon \in(0, C)} A \mathbb{M}^{\alpha}\left(T_{d_{\varepsilon}}(R)\right)<+\infty,
$$

as well as $B \mathbb{M}^{\beta}\left(S_{d_{\varepsilon}}(R)\right)<+\infty$ and since $C>0$, also $\mathbb{M}^{\delta}\left(S_{d_{\varepsilon}}(R)\right)<+\infty$ for all $\varepsilon \in(0, C)$. Therefore, one may let $\varepsilon \rightarrow 0^{+}$in [43, arriving at

$$
\liminf _{\nu} \mathfrak{F}\left(T_{\nu}, S_{\nu}\right) \geqslant \mathfrak{F}\left(T_{d}(R), S_{d}(R)\right),
$$

which shows that the pair $\left(T_{d}(R), S_{d}(R)\right)$ is a minimizer of the functional $\mathfrak{F}$.

CASE $l=0$. In other words, $l_{v}=\mathbb{M}^{\delta}\left(S_{\nu}\right) \rightarrow 0$ as $v \rightarrow \infty$. Since $S_{v} \leqslant R_{\nu}$ and $\partial R_{\nu}=\phi$, and all the currents $R_{\nu}$ are acyclic by construction, while $\delta \leqslant \alpha$ according to the assumptions, Theorem7.4 yields $\mathbb{M}^{\alpha}\left(S_{v}\right) \rightarrow 0$ as $v \rightarrow \infty$. Thus

$$
\begin{aligned}
\liminf _{\nu} \mathfrak{F}\left(T_{v}, S_{v}\right) & =\liminf _{\nu}\left(A \mathbb{M}^{\alpha}\left(T_{v}\right)+B \mathbb{M}^{\beta}\left(S_{v}\right)+H\left(\mathbb{M}^{\delta}\left(S_{v}\right)\right)\right) \\
& \geqslant \liminf _{\nu}\left(A \mathbb{M}^{\alpha}\left(T_{v}\right)+H\left(\mathbb{M}^{\delta}\left(S_{v}\right)\right)\right) \\
& =\liminf _{\nu}\left(A \mathbb{M}^{\alpha}\left(R_{\nu}\right)+H\left(\mathbb{M}^{\delta}\left(S_{v}\right)\right)-A \mathbb{M}^{\alpha}\left(S_{v}\right)\right),
\end{aligned}
$$

and taking into account that

$$
H\left(\mathbb{M}^{\delta}\left(S_{v}\right)\right)-A \mathbb{M}^{\alpha}\left(S_{v}\right)=H\left(l_{v}\right)-A \mathbb{M}^{\alpha}\left(S_{v}\right) \rightarrow 0
$$

as $v \rightarrow \infty$, we get

$$
\liminf _{\nu} \mathfrak{F}\left(T_{\nu}, S_{\nu}\right) \geqslant \liminf _{\nu} A \mathbb{M}^{\alpha}\left(R_{\nu}\right) \geqslant A \mathbb{M}^{\alpha}(R)=\mathfrak{F}(R, 0) .
$$

Therefore, in this case the pair $(R, 0)$ is a minimizer of the functional $\mathfrak{F}$, which concludes the proof. 


\section{Reduction to known problems}

In this section we consider a particular case of Problem 1 with $\alpha=\beta=1$ and $\delta=0$ and show that such a problem is equivalent to the classical problem of finding an optimal transportation network formulated without using the language of Federer-Fleming currents (such a formulation is studied in [9]).

Under the assumptions $\alpha=\beta=1$ and $\delta=0$ Problem 1 can be stated in the following way.

Problem 2 Find a couple $\left(T_{\mathrm{opt}}, S_{\mathrm{opt}}\right)$ of one-dimensional real flat chains minimizing the functional $\mathfrak{F}$ defined by the formula

$$
\mathfrak{F}(T, S)=A \mathbb{M}(T)+B \mathbb{M}(S)+H\left(\mathbb{M}^{0}(S)\right),
$$

among all couples $(T, S)$ of real one-dimensional flat chains of finite mass, satisfying (3).

We now define a new functional $G$ over couples $(\eta, \Sigma)$, where $\eta$ is a transport (i.e. a nonnegative finite Borel measure on $\Theta$ ) and $\Sigma \subset \mathbb{R}^{n}$ is a Borel set. Namely, we set

$$
G(\eta, \Sigma):=\int_{\Theta}\left(A \mathcal{H}^{1}(\theta \backslash \Sigma)+B \mathcal{H}^{1}(\theta \cap \Sigma)\right) \mathrm{d} \eta(\theta)
$$

The meaning of $G(\eta, \Sigma)$ may be explained as follows. Suppose that a single citizen chooses a path $\theta \in \Theta$ in his everyday movement. Assume that $\Sigma$ stands for the transportation network, so that for a citizen choosing the route $\theta$ the cost of using this network would be proportional to $\mathcal{H}^{1}(\theta \cap \Sigma)$ (i.e. to the length of the part of the route made with the help of the network) with coefficient $B \geqslant 0$. For the same citizen, moving without the use of the network by distance $t$ is assumed to cost $A t$ for a given $A \geqslant 0$. Therefore the integrand in (44) gives the individual cost of moving along the route $\theta$. If the transport $\eta$ describes the collective behaviour of the population, so that, heuristically, $\eta(\theta)$ gives the number of people choosing the route $\theta$ in their everyday movements, then $G(\eta, \Sigma)$ gives the total cost of transportation of the population to services or workplaces. Clearly, for $\eta$ to describe the pattern of behaviour of the population in the above sense, one has to require

$$
\eta(0)=\varphi^{+}, \quad \eta(1)=\varphi^{-} .
$$

Clearly, the population as a whole chooses the way of transportation (i.e. the transport $\eta$ ) so as to minimize $G(\cdot, \Sigma)$ among all transports satisfying (45) (further called admissible transports). In other words, the number

$$
\left.M K\left(\varphi^{+}, \varphi^{-}, \Sigma\right):=\inf \{G(\eta, \Sigma): \eta \text { a transport satisfying } 45]\right\}
$$

gives the cost of everyday movement of the population from their places of residence to workplaces and/or services.

We now describe another way of obtaining the same cost $M K\left(\varphi^{+}, \varphi^{-}, \Sigma\right)$ which is more often used in the theory of optimal transportation. Namely, rather than using transports, it is customary to describe the behaviour of the population by so-called transport plans, i.e. by finite positive Borel measures $\gamma$ over $\mathbb{R}^{n} \times \mathbb{R}^{n}$, so that, heuristically, $\gamma(x, y)$ gives the number of people moving from $x$ to $y$. Note that in this sense a transport plans $\gamma$ gives much less information on the movement of the population than the transport $\eta$, namely, it says nothing about the routes people are choosing, but 
just describes the source and destination points of the movement. Clearly, a transport plan $\gamma$ has to satisfy the requirement on marginals

$$
\pi_{\#}^{ \pm} \gamma=\varphi^{ \pm},
$$

where $\pi^{ \pm}\left(x^{+}, x^{-}\right):=x^{ \pm}$(such transport plans will further be called admissible).

Under the assumptions on the cost of movement made above, it is quite reasonable to suppose that each single citizen moving from $x$ to $y$ would choose the route $\theta \in \Theta$ minimizing the total cost of movement, and therefore would spend

$$
d_{\Sigma}(x, y):=\inf \left\{A \mathcal{H}^{1}(\theta \backslash \Sigma)+B \mathcal{H}^{1}(\theta \cap \Sigma): \theta \in \Theta, \theta(0)=x, \theta(1)=y\right\} .
$$

If the behaviour of the population is described by a transport plans $\gamma$ satisfying [46], then the total cost of transportation of the population is given by

$$
\hat{G}(\gamma, \Sigma):=\int_{\mathbb{R}^{n} \times \mathbb{R}^{n}} d_{\Sigma}(x, y) \mathrm{d} \gamma(x, y) .
$$

In [10] it has been shown that the problem of minimizing the cost $G(\cdot, \Sigma)$ among all admissible transport plans is in fact equivalent to that of minimizing the cost $\hat{G}(\cdot, \Sigma)$ among admissible transport plans. The precise meaning of this assertion is given by the statement below.

Proposition 9.1 For each Borel set $\Sigma \subset \mathbb{R}^{n}$ one has

$$
M K\left(\varphi^{+}, \varphi^{-}, \Sigma\right)=\inf \{\hat{G}(\gamma, \Sigma): \gamma \text { a transport plan satisfying } 46\} .
$$

Further, there is an admissible transport $\eta^{\prime}=\eta^{\prime}(\Sigma)$ and a transport plan $\gamma^{\prime}=\gamma^{\prime}(\Sigma)$ (both depending on $\Sigma$ ) such that

$$
M K\left(\varphi^{+}, \varphi^{-}, \Sigma\right)=G\left(\eta^{\prime}, \Sigma\right)=\hat{G}\left(\gamma^{\prime}, \Sigma\right) .
$$

Moreover, $\eta^{\prime}$-a.e. $\theta \in \Theta$ is a simple arc. Finally, if $\eta^{\prime}$ is an admissible transport such that $M K\left(\varphi^{+}, \varphi^{-}, \Sigma\right)=G\left(\eta^{\prime}, \Sigma\right)$, then one can take $\gamma^{\prime}:=\left(p_{0} \times p_{1}\right)_{\#} \eta^{\prime}$. Conversely, there is a Borel measurable function $q: \mathbb{R}^{n} \times \mathbb{R}^{n} \rightarrow \Theta$ such that if $\gamma^{\prime}$ is an admissible transport plan such that $M K\left(\varphi^{+}, \varphi^{-}, \Sigma\right)=\hat{G}\left(\gamma^{\prime}, \Sigma\right)$, then one can take $\eta^{\prime}:=q_{\#} \gamma^{\prime}$.

It is important to mention that since $d_{\Sigma}$ is easily verified to satisfy the triangle inequality, then it is well known that

$$
M K\left(\varphi^{+}, \varphi^{-}, \Sigma\right)=\operatorname{MK}\left(\tilde{\varphi}^{+}, \tilde{\varphi}^{-}, \Sigma\right) \quad \text { whenever } \quad \varphi^{+}-\varphi^{-}=\tilde{\varphi}^{+}-\tilde{\varphi}^{-}
$$

Supposing now that the total cost which determines the transportation network is given by the cost of everyday movement of the population $M K\left(\varphi^{+}, \varphi^{-}, \Sigma\right)$ and of the cost of constructing the network given by $H\left(\mathcal{H}^{1}(\Sigma)\right.$ ) (i.e. depending only on the length $\mathcal{H}^{1}(\Sigma)$ of the network), we get the following natural minimization problem to find the optimal transportation network $\Sigma$.

Problem 3 Find a Borel set $\Sigma_{\text {opt }} \subset \mathbb{R}^{n}$ minimizing the functional

$$
\Sigma \mapsto M K\left(\varphi^{+}, \varphi^{-}, \Sigma\right)+H\left(\mathcal{H}^{1}(\Sigma)\right)
$$

among all Borel sets $\Sigma \subset \mathbb{R}^{n}$. 
In view of the definition of $M K\left(\varphi^{+}, \varphi^{-}, \Sigma\right)$ and Proposition 9.1, we see that each solution $\Sigma_{\text {opt }} \subset \mathbb{R}^{n}$ to Problem 3 together with the corresponding optimal transport $\eta_{\text {opt }}:=\eta^{\prime}\left(\Sigma_{\text {opt }}\right)$ (resp. the optimal transport plan $\left.\gamma_{\mathrm{opt}}:=\gamma^{\prime}\left(\Sigma_{\mathrm{opt}}\right)\right)$ also solves the following problem.

PRoBlem 4 Find a couple $\left(\eta_{\text {opt }}, \Sigma_{\text {opt }}\right)\left(\right.$ resp. $\left.\left(\gamma_{\text {opt }}, \Sigma_{\text {opt }}\right)\right)$ minimizing the functional $F$ (resp. $\left.\hat{F}\right)$ defined by

$$
F(\eta, \Sigma):=G(\eta, \Sigma)+H\left(\mathcal{H}^{1}(\Sigma)\right) \quad\left(\operatorname{resp} . \hat{F}(\gamma, \Sigma):=\hat{G}(\gamma, \Sigma)+H\left(\mathcal{H}^{1}(\Sigma)\right)\right)
$$

among all couples $(\eta, \Sigma)($ resp. $(\gamma, \Sigma)$ ), where $\eta$ is an admissible transport (resp. $\gamma$ is an admissible transport plan) and $\Sigma \subset \mathbb{R}^{n}$ is a Borel set.

Conversely, if a couple $\left(\eta_{\text {opt }}, \Sigma_{\text {opt }}\right)\left(\right.$ resp. $\left.\left(\gamma_{\text {opt }}, \Sigma_{\text {opt }}\right)\right)$ solves the above Problem 4 then $\Sigma_{\text {opt }}$ solves Problem 3 Clearly, a solution $\left(\eta_{\text {opt }}, \Sigma_{\text {opt }}\right)\left(\right.$ resp. $\left.\left(\gamma_{\text {opt }}, \Sigma_{\text {opt }}\right)\right)$ to Problem 4 gives both the optimal transportation network $\Sigma_{\text {opt }}$ and the optimal pattern of behaviour of the population $\eta_{\text {opt }}$ (resp. $\gamma_{\mathrm{opt}}$ ). Note also that once one knows $\eta_{\mathrm{opt}}\left(\right.$ resp. $\gamma_{\mathrm{opt}}$ ), one can find $\gamma_{\mathrm{opt}}\left(\right.$ resp. $\left.\eta_{\mathrm{opt}}\right)$ as indicated in Proposition 9.1 .

We now show that Problem 4 with linear functions $A$ and $B$ is in fact equivalent to Problem 2 in the sense specified by the statement below. For the sake of brevity we will limit ourselves to the case $A \geqslant B$. The case $A<B$ is quite analogous once one observes that under this condition

$$
G(\eta, \Sigma) \geqslant A \int_{\Theta} \mathcal{H}^{1}(\theta) \mathrm{d} \eta=G(\eta, \emptyset),
$$

and every optimal pair $\left(T_{\mathrm{opt}}, S_{\mathrm{opt}}\right)$ solving Problem 2 has $S_{\mathrm{opt}}=0$, since

$$
\mathfrak{F}(T, S)=A \mathbb{M}(T)+B \mathbb{M}(S)+H\left(\mathbb{M}^{0}(S)\right)>A \mathbb{M}(T+S)=\mathfrak{F}(T+S, 0)
$$

whenever $S \neq 0$.

THEOREM 9.2 Let $A \geqslant B$.

(i) Suppose $\left(T_{\mathrm{opt}}, S_{\mathrm{opt}}\right)$ solves Problem 2, while $\mu_{T_{\mathrm{opt}}} \wedge \mu_{S_{\mathrm{opt}}}=0$ (the existence of such an optimal pair is ensured by Proposition 10.3p. Let $\eta:=\eta_{T_{\mathrm{opt}}}+S_{\mathrm{opt}}$ as defined by Theorem 6.3 and let $\Sigma:=\Sigma_{S_{\mathrm{opt}}}$. Then $\Sigma$ solves Problem 3 Further, the couple $(\eta, \Sigma)$ solves Problem 4 with $\varphi^{ \pm}-\varphi^{+} \wedge \varphi^{-}$instead of $\varphi^{ \pm}$. In particular, if $\varphi^{+}$and $\varphi^{-}$are mutually singular, then $(\eta, \Sigma)$ solves Problem 4

(ii) Conversely, let $\left(\eta_{\mathrm{opt}}, \Sigma_{\mathrm{opt}}\right)$ solve Problem 4 Let $R:=T_{\eta_{\mathrm{opt}}}$ as defined by the relationship 88, and let $S:=R\left\llcorner\Sigma_{\mathrm{opt}}, T:=R-S\right.$. Then $(T, S)$ solves Problem 2 .

(iii) Finally, $F\left(\eta_{\text {opt }}, \Sigma_{\text {opt }}\right)=\mathfrak{F}\left(T_{\text {opt }}, S_{\text {opt }}\right)$.

Remark 9.3 It is worth mentioning that once the existence of solutions to Problem 1 (hence in particular to Problem 23 is proven by Theorem 8.1 then the above Theorem 9.2 would give immediately the existence of solutions to Problem 3 .

Proof of Theorem 9.2. Suppose that $\eta$ is an admissible transport such that $\eta$-a.e. $\theta \in \Theta$ is a simple arc (note that by Proposition 9.1 this is the case whenever $(\eta, \Sigma)$ solves Problem 4 ) and $\Sigma \subset \mathbb{R}^{n}$ is a Borel set. Then, letting $R:=T_{\eta}$ as defined by (8), $S:=R\llcorner\Sigma$ and $T:=R-S$, we see that the 
couple $(T, S)$ of flat chains satisfies $(3)$ in view of Theorem 6.2. Further, by the same theorem,

$$
\begin{aligned}
& \mathbb{M}(S)=\mu_{R}(\Sigma) \leqslant \int_{\Theta} \mathbb{M}\left(\llbracket \theta \rrbracket\llcorner\Sigma) \mathrm{d} \eta(\theta)=\int_{\Theta} \mathcal{H}^{1}(\theta \cap \Sigma) \mathrm{d} \eta(\theta),\right. \\
& \mathbb{M}(T)=\mu_{R}\left(\mathbb{R}^{n} \backslash \Sigma\right) \leqslant \int_{\Theta} \mathbb{M}\left(\left[\because \theta \rrbracket\left\llcorner\left(\mathbb{R}^{n} \backslash \Sigma\right)\right) \mathrm{d} \eta(\theta)=\int_{\Theta} \mathcal{H}^{1}(\theta \backslash \Sigma) \mathrm{d} \eta(\theta),\right.\right.
\end{aligned}
$$

and hence $A \mathbb{M}(T)+B \mathbb{M}(S) \leqslant G(\eta, \Sigma)$. On the other hand, $\mathbb{M}^{0}(S) \leqslant \mathcal{H}^{1}(\Sigma)$, and hence $\mathfrak{F}(T, S) \leqslant F(\eta, \Sigma)$.

Suppose now that the couple $(T, S)$ of flat chains of finite mass satisfies (3), and further that $S$ is rectifiable and that both $T, S$ and $T+S$ are acyclic, while $\mu_{T}$ and $\mu_{S}$ are mutually singular (note that according to Theorems 10.1 and 10.2 , the solutions to Problem 2 belong exactly to this class of couples of flat chains). Then $\eta:=\eta_{T+S}$ as defined by Theorem 6.3 satisfies

$$
\begin{aligned}
& \eta(0)=(\partial(T+S))^{+}=\varphi^{+}-\varphi^{+} \wedge \varphi^{-}, \\
& \eta(1)=(\partial(T+S))^{-}=\varphi^{-}-\varphi^{+} \wedge \varphi^{-} .
\end{aligned}
$$

Let $\Sigma:=\Sigma_{S}$ as defined by Lemma7.1. Then $\mu_{S}$ is concentrated on $\Sigma$. Hence, setting $R:=T+S$, one gets $S=R\left\llcorner\Sigma, T=R\left\llcorner\left(\mathbb{R}^{n} \backslash \Sigma\right)\right.\right.$. Letting $\eta$ be such that $R=T_{\eta}$ as defined by Theorem 6.3. we deduce from Proposition 6.8 that

$$
\begin{aligned}
& \mathbb{M}(S)=\mu_{R}(\Sigma)=\int_{\Theta} \mathcal{H}^{1}(\theta \cap \Sigma) \mathrm{d} \eta(\theta), \\
& \mathbb{M}(T)=\mu_{R}\left(\mathbb{R}^{n} \backslash \Sigma\right)=\int_{\Theta} \mathcal{H}^{1}(\theta \backslash \Sigma) \mathrm{d} \eta(\theta),
\end{aligned}
$$

and hence $G(\eta, \Sigma)=A \mathbb{M}(T)+B \mathbb{M}(S)$. Further, from the definition of $\Sigma$ one has $\mathbb{M}^{0}(S)=$ $\mathcal{H}^{1}(\Sigma)$, and hence $F(\eta, \Sigma)=\mathfrak{F}(T, S)$, which concludes the proof.

\section{Qualitative properties of optimal currents}

Here and below we always suppose the existence of a couple $(T, S)$ of real one-dimensional flat chains of finite mass satisfying (3) such that $\mathfrak{F}(T, S)<+\infty$ (which means that the minimization Problem 1 is nontrivial). We further also suppose that either the penalization function $H$ is concave, or $\delta=0$.

\subsection{Acyclicity}

One may now state the following easy result.

THEOREM 10.1 Let $(T, S)$ be a pair of one-dimensional real flat chains of finite mass. Then there is a pair $\left(T^{\prime}, S^{\prime}\right)$ of acyclic currents such that $T^{\prime} \leqslant T, S^{\prime} \leqslant S, \mathfrak{F}\left(T^{\prime}, S^{\prime}\right) \leqslant \mathfrak{F}(T, S)$ and $\partial\left(T^{\prime}+S^{\prime}\right)=$ $\partial(T+S)$. Moreover,

(i) if $A>0$ and $T$ is not acyclic, then $\mathfrak{F}\left(T^{\prime}, S^{\prime}\right)<\mathfrak{F}(T, S)$;

(ii) if either $B>0$ or $H$ is strictly increasing, and $S$ is not acyclic, then $\mathfrak{F}\left(T^{\prime}, S^{\prime}\right)<\mathfrak{F}(T, S)$;

(iii) if $A>0$, and either $B>0$ or $H$ is strictly increasing, while $T+S$ is not acyclic, then $T^{\prime}+S^{\prime}$ is acyclic, and $\mathfrak{F}\left(T^{\prime}, S^{\prime}\right)<\mathfrak{F}(T, S)$. 
In particular, if the pair $(T, S)$ solves Problem 1 , then $A>0$ implies acyclicity of $T$, and either $B>0$ or strict monotonicity of $H$ imply acyclicity of $S$, while if both $A>0$, and either $B>0$ or $H$ is strictly increasing, then $T+S$ is acyclic.

Proof. If $T$ (resp. $S$ ) is acyclic, it is enough to set $T^{\prime}:=T$ (resp. $S^{\prime}:=S$ ). Otherwise in view of Proposition 3.12 $T$ (resp. $S$ ) contains a cycle $C \neq 0$ such that the current $T^{\prime}:=T-C$ (resp. $\left.S^{\prime}:=S-C\right)$ is acyclic. By Lemma 3.7 one has $\mathbb{M}^{\lambda}\left(T^{\prime}\right)<\mathbb{M}^{\lambda}(T)\left(\right.$ resp. $\left.\mathbb{M}^{\lambda}\left(S^{\prime}\right)<\mathbb{M}^{\lambda}(S)\right)$ for all $\lambda \in(0,1]$. Therefore, as $\partial T^{\prime}=\partial T$ (resp. $\partial S^{\prime}=\partial S$ ), we get $\partial\left(T^{\prime}+S^{\prime}\right)=\partial(T+S)$, and $\mathfrak{F}\left(T^{\prime}, S^{\prime}\right) \leqslant \mathfrak{F}(T, S)$ (with strict inequality in cases (i) and (ii)).

We now prove (iii). According to Lemma 4.6 one may assume without loss of generality that $\mu_{T} \wedge \mu_{S}=0$, i.e. there is a Borel set $E \subset \mathbb{R}^{n}$ such that

$$
T=T\left\llcorner E, \quad S=S\left\llcorner\left(\mathbb{R}^{n} \backslash E\right) .\right.\right.
$$

If $T+S$ is not acyclic, then by Proposition 3.12 there is a cycle $C \neq 0, C \leqslant T+S$, such that the current $T+S-C$ is acyclic. Denoting

$$
T^{\prime}:=T-C\left\llcorner E, \quad S^{\prime}:=S-C\left\llcorner\left(\mathbb{R}^{n} \backslash E\right),\right.\right.
$$

we get $T^{\prime}+S^{\prime}=T+S-C$, and hence $\partial\left(T^{\prime}+S^{\prime}\right)=\partial(T+S)$. On the other hand, $C \leqslant T+S$ implies

$$
C\llcorner E \leqslant(T+S)\llcorner E=T .
$$

By Remark 3.6 one has $T^{\prime} \leqslant T$. In the same way, $S^{\prime} \leqslant S$, and hence, in view of Lemma 3.7. $\mathfrak{F}\left(T^{\prime}, S^{\prime}\right)<\mathfrak{F}(T, S)$.

It is worth remarking that with the help of Theorem 7.4 one can easily find estimates on the masses of solutions to Problem 1 .

\subsection{Rectifiability}

The following result is an easy consequence of Theorem 2.1

THEOREM 10.2 Let $(T, S)$ be a pair of real one-dimensional flat chains of finite mass satisfying $\mathfrak{F}(T, S)<+\infty$. Then the following assertions hold:

(i) if $A \neq 0$ and $\alpha<1$, then $T$ is rectifiable;

(ii) if either $B \neq 0$ and $\beta<1$, or $H$ is unbounded and $\delta<1$, then $S$ is rectifiable.

In particular, the above assertions are valid for every optimal pair solving Problem 1

Proof. We have $\mathfrak{F}(T, S)<+\infty$. When $A \neq 0$ one therefore has $\mathbb{M}^{\alpha}(T)<+\infty$, and hence (i) follows from Theorem 2.1. Analogously, if $B \neq 0$, then $\mathbb{M}^{\beta}(S)<+\infty$, so that rectifiability of $S$ follows from Theorem 2.1 when $\beta<1$. Finally, if $H$ is unbounded, then $\mathbb{M}^{\delta}(S)<+\infty$, and hence rectifiability of $S$ follows again from Theorem 2.1 when $\delta<1$.

\subsection{Properties of support}

We first show the existence of minimizing couples $(T, S)$ solving Problem 1 such that $T$ and $S$ are concentrated on disjoint sets. Here and below solvability of Problem 11 will always be tacitly assumed. 
Proposition 10.3 There is a minimizing couple $(T, S)$ solving Problem 1 such $\mu_{T} \wedge \mu_{S}=0$. In particular, $\mathcal{H}^{1}\left(\Sigma_{T} \cap \Sigma_{S}\right)=0$. Further, if either of the conditions (i)-(v) of Lemma 4.7 holds, then the above property is true for every minimizing couple $(T, S)$ solving Problem 1

Proof. Follows immediately from Lemma 4.6

We are now able to prove the following assertion which says that whenever the penalization $H$ is concave, then there is an optimal pair $(T, S)$ of flat chains solving Problem 1 as follows: there is a threshold $d \geqslant 0$ such that $T$ is concentrated on $\left\{x: \theta_{T+S}<d\right\}$, while $S$ is concentrated on the set $\left\{x: \theta_{T+S} \geqslant d\right\}$, and in certain cases every optimal pair satisfies such a condition. In other words, recalling that $S$ stands for the flow of people using the transportation network, while $T$ stands for that of people moving by their own means, it means that the transportation network has to be constructed in the set of points where the density $\theta_{T+S}$ of the total flow of people is greater than the threshold $d$.

THEOREM 10.4 (Bathtub principle) Assume that $H$ is concave and unbounded, and let $\left(T^{\prime}, S^{\prime}\right)$ be any solution to Problem 1 having $\mu_{T^{\prime}} \wedge \mu_{S^{\prime}}=0$ (once Problem 1 is solvable, the existence of such a pair is guaranteed by Proposition 10.3 . Then there exists an optimal pair $(T, S)$ of flat chains solving Problem 1 such that $\mu_{T} \wedge \mu_{S}=0$ and under either of the conditions (i)-(iv) of Lemma10.6 (with $C:=H_{-}^{\prime}\left(\mathbb{M}^{\delta}\left(S^{\prime}\right)\right.$ ), where $H_{-}^{\prime}$ stands for the left derivative of $H$ ),

$$
\begin{array}{ll}
\theta_{S}(x)=\theta_{T+S}(x) \geqslant d & \text { for } \mathcal{H}^{1} \text {-a.e. } x \in \Sigma_{T+S} \\
\theta_{T}(x)=\theta_{T+S}(x)<d & \text { for } \mathcal{H}^{1} \text {-a.e. } x \in \Sigma_{T+S}
\end{array}
$$

for some constant $d \geqslant 0$ (moreover, $d>0$ under either of the conditions ( $\left.\mathrm{i}^{\prime}\right)$-(iii') of Lemma 10.6 below). In particular, if $S$ is rectifiable, then $S$ is concentrated on $\left\{x: \theta_{T+S}(x) \geqslant d\right\}$ and $T$ is concentrated on the set $\left\{x: \theta_{T+S}(x)<d\right\}$. Moreover, the above properties hold for all optimal pairs $(T, S)$ satisfying $\mu_{T} \wedge \mu_{S}=0$ if in addition one assumes that $H$ is strictly concave.

Proof. We first note that we may restrict ourselves to the case $S^{\prime} \neq 0$, since otherwise it is enough to choose $T=T^{\prime}, S=S^{\prime}$ and $d:=\mathbb{M}(\partial T) / 2$ in view of Theorem 7.3 Now the existence of an optimal pair of flat chains solving Problem 1 follows immediately from Lemma 10.5 below (for the assertion in the case of rectifiable $S$ one has just to note that in this case one may consider $S$ concentrated on $\Sigma_{S} \subset \Sigma_{T+S}$ ).

The following assertions have been used in the above proof.

LEMMA 10.5 Let $(T, S), S \neq 0$, be a pair of flat chains of finite mass satisfying $\mu_{T} \wedge \mu_{S}=0$, and suppose the function $H$ is concave. Under either of the conditions (i)-(iv) (resp. (i')-(iii')) of Lemma 10.6 there is a constant $d \geqslant 0$ (resp. $d>0$ ), depending only on $\alpha, \beta, \delta, A, B$ and $C \in$ $\left[H_{+}^{\prime}\left(\mathbb{M}^{\delta}(S)\right), H_{-}^{\prime}\left(\mathbb{M}^{\delta}(S)\right)\right]$, where $H_{ \pm}^{\prime}$ stand for the left and right derivatives of $H$ respectively, and there is a pair of flat chains $\left(T^{\prime}, S^{\prime}\right)$, such that $T^{\prime}+S^{\prime}=T+S, \mu_{T^{\prime}} \wedge \mu_{S^{\prime}}=0, \mathfrak{F}\left(T^{\prime}, S^{\prime}\right) \leqslant \mathfrak{F}(T, S)$, and

$$
\begin{array}{ll}
\theta_{S^{\prime}}(x)=\theta_{T^{\prime}+S^{\prime}}(x) \geqslant d & \text { for } \mathcal{H}^{1} \text {-a.e. } x \in \Sigma_{T^{\prime}+S^{\prime}}, \\
\theta_{T^{\prime}}(x)=\theta_{T^{\prime}+S^{\prime}}(x)<d & \text { for } \mathcal{H}^{1} \text {-a.e. } x \in \Sigma_{T^{\prime}+S^{\prime}} .
\end{array}
$$

Moreover, if $T$ (resp. $S$ ) is rectifiable, then so is $T^{\prime}$ (resp. $S^{\prime}$ ).

Furthermore, if the function $H$ is strictly concave, and one of the properties (48) does not hold for $T, S$ in place of $T^{\prime}, S^{\prime}$, then one can find a pair $\left(T^{\prime}, S^{\prime}\right)$ as above with $\mathfrak{F}\left(T^{\prime}, S^{\prime}\right)<\mathfrak{F}(T, S)$. 
Proof. In view of the assumption on $C$ we have

$$
H\left(\mathbb{M}^{\delta}(S)+t\right) \leqslant H\left(\mathbb{M}^{\delta}(S)\right)+C t \quad \text { for all } t \geqslant-\mathbb{M}^{\delta}(S),
$$

with strict inequality when $t \neq 0$ and $H$ is strictly concave (note that in the latter case $C \neq 0$ in view of the strict concavity of $H$ ). Consider the function $f: \mathbb{R}^{+} \rightarrow \mathbb{R}$ defined by the formula $f(t):=A t^{\alpha}-B t^{\beta}-C t^{\delta}$. By Lemma 10.6 there is a $d \geqslant 0$ such that $f(t)>0$ when $t>d$, and $f(t)<0$ when $t \in(0, d)$. Moreover, $d>0$ if either of conditions (i')-(iii') of Lemma 10.6 holds. Consider the sets

$$
\Sigma^{+}:=\left\{x \in \Sigma_{T+S}: \theta_{T}(x) \geqslant d\right\}, \quad \Sigma^{-}:=\left\{x \in \Sigma_{T+S}: 0<\theta_{S}(x)<d\right\}
$$

(with $\Sigma^{-}:=\emptyset$ if $d=0$ ). Since the densities $\theta_{T}$ and $\theta_{S}$ are Borel functions, $\Sigma^{ \pm}$are Borel sets so that the currents

$$
\begin{aligned}
R^{+}=T\left\llcorner\Sigma^{+},\right. & R^{-}=S\left\llcorner\Sigma^{-},\right. \\
T^{\prime}=T-R^{+}+R^{-}, & S^{\prime}=S-R^{-}+R^{+}
\end{aligned}
$$

are well defined, and $T^{\prime}+S^{\prime}=T+S$. Observe that the rectifiablity of $T^{\prime}$ (resp. $S^{\prime}$ ) follows from that of $T$ (resp. $S$ ) since both $R^{+}$and $R^{-}$are rectifiable in view of Lemma 7.1. We now show that $\mathfrak{F}\left(T^{\prime}, S^{\prime}\right) \leqslant \mathfrak{F}(T, S)$, with strict inequality if $H$ is strictly concave, and one of the properties 48) does not hold for $T, S$ in place of $T^{\prime}, S^{\prime}$ (all the other announced properties of the pair $\left(T^{\prime}, S^{\prime}\right)$ are immediate). Since $R^{+} \leqslant T, R^{-} \leqslant S$, while $\mu_{T} \wedge \mu_{S}=0$, we have

$$
\begin{aligned}
\mathbb{M}^{\alpha}\left(T^{\prime}\right) & =\mathbb{M}^{\alpha}\left(T-R^{+}+R^{-}\right)=\mathbb{M}^{\alpha}(T)-\mathbb{M}^{\alpha}\left(R^{+}\right)+\mathbb{M}^{\alpha}\left(R^{-}\right), \\
\mathbb{M}^{\beta}\left(S^{\prime}\right) & =\mathbb{M}^{\beta}\left(S-R^{-}+R^{+}\right)=\mathbb{M}^{\beta}(S)-\mathbb{M}^{\beta}\left(R^{-}\right)+\mathbb{M}^{\beta}\left(R^{+}\right), \\
\mathbb{M}^{\delta}\left(S^{\prime}\right) & =\mathbb{M}^{\delta}\left(S-R^{-}+R^{+}\right)=\mathbb{M}^{\delta}(S)-\mathbb{M}^{\delta}\left(R^{-}\right)+\mathbb{M}^{\delta}\left(R^{+}\right),
\end{aligned}
$$

and therefore

$$
\begin{aligned}
\mathfrak{F}\left(T^{\prime}, S^{\prime}\right) \leqslant & \mathfrak{F}(T, S)-A \mathbb{M}^{\alpha}\left(R^{+}\right)+A \mathbb{M}^{\alpha}\left(R^{-}\right) \\
& -B \mathbb{M}^{\beta}\left(R^{-}\right)+B \mathbb{M}^{\beta}\left(R^{+}\right)-C \mathbb{M}^{\delta}\left(R^{-}\right)+C \mathbb{M}^{\delta}\left(R^{+}\right) .
\end{aligned}
$$

Recalling that by Lemma 7.1 the sets $\Sigma^{ \pm}$are countably $\left(\mathcal{H}^{1}, 1\right)$-rectifiable, we get

$$
\begin{aligned}
\mathfrak{F}\left(T^{\prime}, S^{\prime}\right) \leqslant & \mathfrak{F}(T, S)-\int_{\Sigma^{+}}\left(A \theta_{T}^{\alpha}(x)-B \theta_{T}^{\beta}(x)-C \theta_{T}^{\delta}(x)\right) \mathrm{d} \mathcal{H}^{1}(x) \\
& +\int_{\Sigma^{-}}\left(A \theta_{S}^{\alpha}(x)-B \theta_{S}^{\beta}(x)-C \theta_{S}^{\delta}(x)\right) \mathrm{d} \mathcal{H}^{1}(x) \\
= & \mathfrak{F}(T, S)-\int_{\Sigma^{+}} f\left(\theta_{T}(x)\right) \mathrm{d} \mathcal{H}^{1}(x)+\int_{\Sigma^{-}} f\left(\theta_{S}(x)\right) \mathrm{d} \mathcal{H}^{1}(x) \leqslant \mathfrak{F}(T, S) .
\end{aligned}
$$

Moreover, if the first property of (48) does not hold for $T, S$ in place of $T^{\prime}, S^{\prime}$, then $\int_{\Sigma^{-}} f\left(\theta_{S}(x)\right) \mathrm{d} \mathcal{H}^{1}(x)<0$, and hence the second inequality of $(50)$ is strict. Further, if the second property of (48) does not hold for $T, S$ in place of $T^{\prime}, S^{\prime}$, then either

$$
\mathcal{H}^{1}\left(\left\{x \in \Sigma_{T+S}: \theta_{T}(x)>d\right\}\right)>0,
$$


or else

$$
\mathcal{H}^{1}\left(\left\{x \in \Sigma_{T+S}: \theta_{T}(x)=d\right\}\right)>0 .
$$

In the former case $\int_{\Sigma^{+}} f\left(\theta_{T}(x)\right) \mathrm{d} \mathcal{H}^{1}(x)>0$, and hence again the second inequality of 50 is strict. In the latter case one still has $R^{+} \neq 0$, and we may consider without loss of generality $\mathcal{H}^{1}\left(\Sigma^{-}\right)=0$ (since otherwise the strict inequality $\mathfrak{F}\left(T^{\prime}, S^{\prime}\right)<\mathfrak{F}(T, S)$ has already been proven), so that $R^{-}=0$. Therefore the inequality in (49) becomes strict when $H$ is strictly concave (because $\mathbb{M}^{\delta}\left(R^{+}\right) \neq 0$ ), and hence the first inequality of (50) is strict, which concludes the proof.

Lemma 10.6 Let $f:[0,+\infty) \rightarrow \mathbb{R}$ be defined by

$$
f(t):=A t^{\alpha}-B t^{\beta}-C t^{\delta}
$$

with $\alpha, \beta, \delta \in[0,1]$ and $A, B, C \geqslant 0$. Suppose also that either of the following conditions hold:

(i) $\alpha>\beta \vee \delta$;

(ii) $\alpha=\beta>\delta$ and $A>B$;

(iii) $\alpha=\delta>\beta$ and $A>C$;

(iv) $\alpha=\beta=\delta$ and $A>B+C$.

Then there is a $d \geqslant 0$ such that

$$
f(t) \geqslant 0 \quad \text { if and only if } t \geqslant d \text { or } t=0 .
$$

Moreover, $d>0$ under either of the following conditions:

(i') (i) holds and either $B \neq 0$ or $C \neq 0$;

(ii') (ii) holds and $C \neq 0$;

(iii') (iii) holds and $B>0$.

Proof. Case (i). Suppose $\beta \geqslant \delta$ (the other case being symmetric). Dividing by $t^{\delta}$, we get

$$
f(t) \geqslant 0 \text { if and only if } g(s):=A s^{\sigma}-B s-C \geqslant 0,
$$

where $s:=t^{\beta-\delta}, \sigma:=(\alpha-\delta) /(\beta-\delta)$. Noticing that $\sigma>1$ in the case we are considering, we see that the derivative $g^{\prime}(s)=A \sigma s^{\sigma-1}-B$ is nondecreasing (resp. strictly increasing if $A>0$ ). Hence, $g$ is convex (resp. strictly convex), and as $g(0)=-C \leqslant 0$ and $g^{\prime}(0)=-B \leqslant 0$, we get the existence of some $\bar{s} \geqslant 0$ such that $g(s) \geqslant 0$ if and only if $s \geqslant \bar{s}$, while $\bar{s}>0$ if either $C \geqslant 0$ or $B \geqslant 0$. It is then enough to set $d:=\bar{s}^{1 /(\beta-\delta)}$.

Case (ii). Dividing by $t^{\delta}$, we infer that $f(t) \geqslant 0$ if and only if

$$
(A-B) t^{\alpha / \delta}-C \geqslant 0
$$

which means that one may take $d:=C^{\delta / \alpha} /(A-B)$.

Case (iii). This is completely analogous to case (ii).

Case (iv). Dividing by $t^{\delta}$, we find that $f(t) \geqslant 0$ if and only if

$$
(A-B-C) t^{\alpha} \geqslant 0,
$$

which means $d=0$. 
Now we are able to prove that under natural conditions on problem data, the optimal current $S$ can be chosen to be concentrated on a closed set.

THEOREM 10.7 Suppose that $H$ is concave and unbounded and condition (ii) of Theorem 10.2 holds. Under either of the conditions (i) or (ii) of Lemma 10.6 there exists an optimal pair $(T, S)$ solving Problem 1 such that $S$ is a rectifiable current representable as $S=\theta \llbracket \Sigma \rrbracket$, where $\Sigma \subset \mathbb{R}^{n}$ is a closed countably $\left(\mathcal{H}^{1}, 1\right)$-rectifiable set and $\theta \in L^{1}\left(\mathcal{H}^{1}\llcorner\Sigma)\right.$ is u.s.c. with $\theta(x) \geqslant d$ for $\mathcal{H}^{1}$-a.e. $x \in \Sigma$ and for some $d>0$. Further, if either of the conditions (i)-(v) of Lemma 4.7/holds, while $H$ is strictly concave, then the above assertions are true for all optimal pairs $(T, S)$ solving Problem 1 .

Proof. Let the pair $\left(T^{\prime}, S^{\prime}\right)$ solve Problem 1, while $\mu_{T^{\prime}} \wedge \mu_{S^{\prime}}=0$ (once Problem 1 is solvable, the existence of such a pair is guaranteed by Proposition 10.3 . We may assume $S^{\prime} \neq 0$ (otherwise there is nothing to prove, since we may take, for instance, $S:=S^{\prime}, \theta \equiv 1$ and $\left.\Sigma:=\emptyset\right)$. Note now that since the assumptions (i) or (ii) of Lemma 10.6 hold, while $C:=H_{-}^{\prime}\left(\mathbb{M}^{\delta}\left(S^{\prime}\right)\right)>0$ in view of the assumptions on $H$, by Theorem 10.4 combined with Theorem 10.2 we know that there exists a $d>0$ and an optimal pair $(T, S)$ solving Problem 1 and satisfying $\mu_{T} \wedge \mu_{S}=0$, such that $S$ is rectifiable and concentrated on the set $\Sigma_{S}=\left\{x: \theta_{T+S}(x) \geqslant d\right\}$.

Let $\eta$ be given by Theorem 6.3 so that $T+S=T_{\eta}$. By Lemma 7.2 (ii), taking into account the acyclicity of $T+S$ one has

$$
\mathcal{H}^{1}\left(\left\{x: \theta_{T+S}(x) \neq a_{\eta}(x)\right\}\right)=0 .
$$

Hence $\mathcal{H}^{1}\left(\Sigma_{S} \triangle \Sigma\right)=0$ where $\Sigma:=\left\{x \in \mathbb{R}^{n}: a_{\eta}(x) \geqslant d\right\}$, and $\Sigma$ is closed since $a_{\eta}$ is u.s.c. by Lemma 7.2(i). Therefore $S=a_{\eta} \llbracket \Sigma \rrbracket$, as desired. To conclude the proof, it remains to observe that under either of the conditions (i)-(v) of Lemma 4.7 every optimal pair $(T, S)$ solving Problem 1 satisfies $\mu_{T} \wedge \mu_{S}=0$, while if $H$ is strictly concave, then every such pair will have $S$ concentrated on $\left\{x: \theta_{T+S}(x) \geqslant d\right\}$, and hence will satisfy the desired conditions.

It is worth mentioning that Theorem 10.7 is only valid under concavity assumptions on $H$. In fact, in [9] it has been shown that even when $A=1, B=0$, but

$$
H(t):= \begin{cases}0, & t \leqslant l \\ +\infty, & \text { otherwise }\end{cases}
$$

then for some measures $\varphi^{+}, \varphi^{-}$Problem 3 may admit no solutions $\Sigma$ which are closed sets. In view of Theorem 9.2 this means that no solution $(T, S)$ to Problem 1 with $\alpha=\beta=1, \delta=0$ and $A, B$ and $H$ as above has the property stated in Theorem 10.7 i.e. $S=\theta \llbracket \Sigma \rrbracket$, where $\Sigma \subset \mathbb{R}^{n}$ is a closed countably $\left(\mathcal{H}^{1}, 1\right)$-rectifiable set, and $\theta(x)>0$ for $\mathcal{H}^{1}$-a.e. $x \in \Sigma$.

\section{REFERENCES}

1. Ambrosio, L. Lecture notes on optimal transport problems. Mathematical Aspects of Evolving Interfaces, Lecture Notes in Math. 1812, Springer (2003), 1-52. Zbl 1047.35001 MR 2011032

2. Ambrosio, L., Fusco, N., \& Pallara, D. Functions of Bounded Variation and Free Discontinuity $\begin{array}{lll}\text { Problems. } & \text { Oxford Math. Monographs, Oxford Univ. Press, Oxford (2000). Zbl } 0957.49001\end{array}$ MR 1857292

3. Ambrosio, L., \& Kirchheim, B. Currents in metric spaces. Acta Math. 185 (2000), 1-80. Zbl 0984.49025 MR 1794185

4. Bernot, M., Caselles, V., \& Morel, J.-M. Are there infinite irrigation trees? J. Math. Fluid Mech., to appear. 
5. Bernot, M., Caselles, V., \& Morel, J.-M. Traffic plans. Publ. Mat. 49 (2005), 417-451. Zbl pre02233222 MR 2177636

6. Bhaskaran, S., \& Salzborn, F. J. M. Optimal design of gas pipeline networks. J. Oper. Res. Soc. 30 (1979), 1047-1060. Zbl 0422.90046

7. Brenier, Y. The dual least action problem for an ideal, incompressible fluid. Arch. Ration. Mech. Anal. 122 (1993), 323-351. Zbl 0797.76006 MR 1217592

8. Buttazzo, G., \& Bouchitté, G. Characterization of optimal shapes and masses through MongeKantorovich equation. J. Eur. Math. Soc. 3 (2001), 139-168. Zbl 0982.49025 MR 1831873

9. Buttazzo, G., Pratelli, A., Solimini, S., \& Stepanov, E. Mass transportation and urban planning problems. Forthcoming.

10. Buttazzo, G., Pratelli, A., \& Stepanov, E. Optimal pricing policies for public transportation networks. SIAM J. Optim. 16 (2006), 826-853. Zbl 1093.49030 MR 2197558

11. Caselles, V., \& Morel, J.-M. Irrigation. Variational Models for Discontinuous Structures (Trieste, 2001), F. Tomarelli and G. Dal Maso (eds.), Progr. Nonlinear Differential Equations Appl. 51, Birkhäuser (2002), 81-90. Zbl 1046.76038 MR 2197839

12. Castaing, C., \& Valadier, M. Convex Analysis and Measurable Multifunctions. Lecture Notes in Math. 580, Springer, Berlin (1977). Zbl 0346.46038 MR 0467310

13. De Pauw, T., \& Hardt, R. Size minimization and approximating problems. Calc. Var. Partial Differential Equations 17 (2003), 405-442. Zbl 1022.49026 MR 1993962

14. Gilbert, E. N. Minimum cost communication networks. Bell System Tech. J. 46 (1967), 2209-2227.

15. LEE, D. H. Low cost drainage networks. Networks 6 (1976), 351-371. Zbl 0348.90061

16. Maddalena, F., Solimini, S., \& Morel, J.-M. A variational model of irrigation patterns. Interfaces Free Bound. 5 (2003), 391-415. Zbl 1057.35076 MR 2031464

17. Morgan, F. Geometric Measure Theory. A Beginner's Guide. Academic Press (1988). Zbl 0671.49043 MR 0933756

18. Simon, L. Lectures on Geometric Measure Theory. Proc. Centre Math. Anal. 3, Austral. Nat. Univ. (1983). Zbl 0546.49019 MR 0756417

19. Smirnov, S. K. Decomposition of solenoidal vector charges into elementary solenoids and the structure of normal one-dimensional currents. St. Petersburg Math. J. 5 (1994), 841-867. Zbl 0832.49024 MR 1246427

20. SRivastava, S. M. A Course on Borel Sets. Grad. Texts in Math. 180, Springer, New York (1998). Zbl 0903.28001 MR 1619545

21. White, B. The deformation theorem for flat chains. Acta Math. 183 (1999), 255-271. Zbl 0980.49035 MR 1738045

22. White, B. Rectifiability of flat chains. Ann. of Math. 150 (1999), 165-184. Zbl 0965.49024 MR 1715323

23. XiA, Q. Optimal paths related to transport problems. Comm. Contemp. Math. 5 (2003), 251-279. Zbl 1032.90003 MR 1966259

24. XIA, Q. Boundary regularity of optimal transport paths. Preprint, 2004. http://www.ma.utexas.edu/ qulxia/.

25. XIA, Q. Interior regularity of optimal transport paths. Calc. Var. Partial Differential Equations 20 (2004), 283-299. Zbl 1080.90008 MR 2062945 